\title{
Usage Monitoring of Electrical Devices in a Smart Home
}

by

Saba Rahimi

A thesis submitted to the Faculty of Graduate and Postdoctoral Affairs in partial fulfillment of the requirements for the degree of Masters of Applied Science in Biomedical Engineering

(Ottawa-Carleton Institute for Biomedical Engineering (OCIBME))

Department of Systems and Computer Engineering

Carleton University

Ottawa, Ontario, Canada, K1S 5B6

January, 2012

(C) Copyright 2012, Saba Rahimi 
Library and Archives

Canada

Published Heritage

Branch

395 Wellington Street

Ottawa ON K1A ON4

Canada
Bibliothèque et

Archives Canada

Direction du

Patrimoine de l'édition

395 , rue Wellington

Ottawa ON K1A ON4

Canada
Your file Votre référence

ISBN: 978-0-494-87804-0

Our file Notre référence

ISBN: $978-0-494-87804-0$

\section{NOTICE:}

The author has granted a nonexclusive license allowing Library and Archives Canada to reproduce, publish, archive, preserve, conserve, communicate to the public by telecommunication or on the Internet, loan, distrbute and sell theses worldwide, for commercial or noncommercial purposes, in microform, paper, electronic and/or any other formats.

The author retains copyright ownership and moral rights in this thesis. Neither the thesis nor substantial extracts from it may be printed or otherwise reproduced without the author's permission.
AVIS:

L'auteur a accordé une licence non exclusive permettant à la Bibliothèque et Archives Canada de reproduire, publier, archiver, sauvegarder, conserver, transmettre au public par télécommunication ou par l'Internet, prêter, distribuer et vendre des thèses partout dans le monde, à des fins commerciales ou autres, sur support microforme, papier, électronique et/ou autres formats.

L'auteur conserve la propriété du droit d'auteur et des droits moraux qui protege cette thèse. $\mathrm{Ni}$ la thèse ni des extraits substantiels de celle-ci ne doivent être imprimés ou autrement reproduits sans son autorisation.
In compliance with the Canadian Privacy Act some supporting forms may have been removed from this thesis.

While these forms may be included in the document page count, their removal does not represent any loss of content from the thesis.
Conformément à la loi canadienne sur la protection de la vie privée, quelques formulaires secondaires ont été enlevés de cette thèse.

Bien que ces formulaires aient inclus dans la pagination, il n'y aura aucun contenu manquant. 


\section{Abstract}

Profiling the usage of electrical devices within a smart home can be used as a method for determining an occupant's activities of daily living, which can support independent living of older adults. A nonintrusive load monitoring (NILM) system monitors the electrical consumption at a single electrical source and the operating schedules of individual devices are determined by disaggregating the composite electrical consumption waveforms. Nonintrusive load monitoring systems are able to detect the status of electrical devices based on the analysis of load signatures, the unique electrical behaviour of an individual device when it is in operation. This work uses a feature-based model, applying steady-state features (i.e., real power and reactive power) and transient features (i.e., switching on transient waveforms), for describing the load signatures of individual electrical devices. In this thesis, a NILM system is presented, which employs several common household electrical devices for data acquisition. Promising results are shown which demonstrate that real power and reactive power are useful steady-state features to identify electrical devices. Furthermore, experimental results showed that different electrical devices can be distinguished based on their switching on transient waveforms. Experimental results showed that the proposed approach can achieve high device recognition accuracy for electrical devices operating individually and simultaneously. 


\section{To my parents}

Mahin Safari \& Najaf Rahimi 


\section{Acknowledgements}

I would like to gratefully acknowledge my supervisors, Dr. Adrian Chan and Dr. Rafik Goubran for their guidance and support throughout my graduate career and during the completion of this thesis. This study was supported by the Natural Sciences and Engineering Research Council of Canada. I am obliged to NSERC, the Department of Systems and Computer Engineering, the Faculty of Graduate Studies, and my supervisors.

I also extend my appreciation to all those authors contributed to my thoughts, opinions, and beliefs. I would like to thank Aida, a loving sister and incredible kind roommate. Thanks to my friends Behrouz and Behnam for their steadfast support. 


\section{Table of Contents}

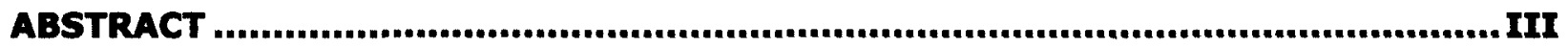

ACKNOWLEDGEMENTS ...................................................................................... V

TABLE OF CONTENTS .......................................................................................... VI

LIST OF TABLES ............................................................................................VIII

LIST OF FIGURES ..............................................................................................IX

LIST OF ABBREVIATIONS ................................................................................XI

1 INTRODUCTION ........................................................................................ 1

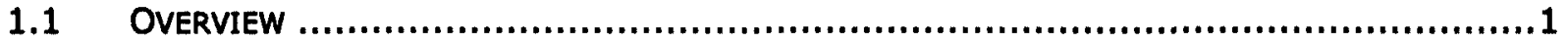

1.1.1 Introduction to Smart Homes ................................................. 1

1.1.2 Load Monitoring Concept....................................................... 4

1.1.3 Types of Load Monitoring Techniques ........................................5

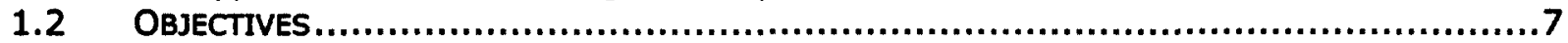

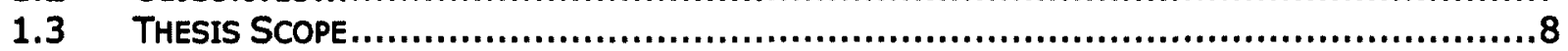

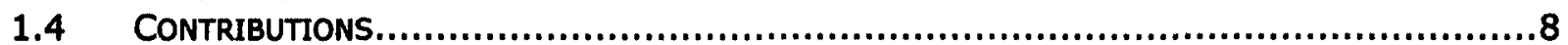

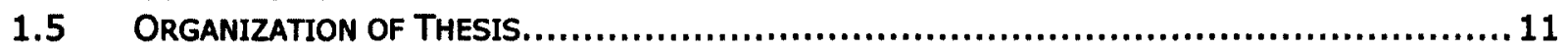

2 BACKGROUND AND LITERATURE REVIEW.................................................. 12

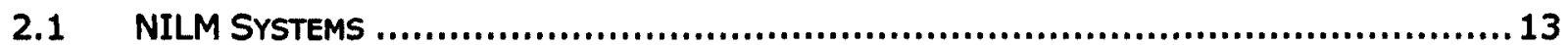

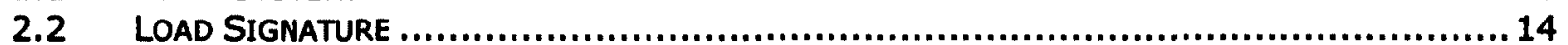

2.2.1 Steady-State Signature ....................................................... 16

2.2.2 Transient Signature.............................................................. 19

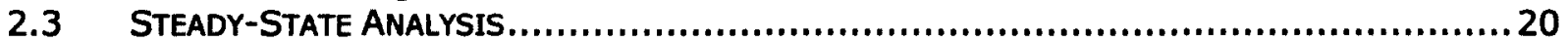

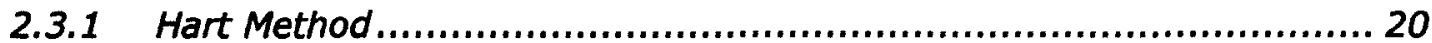

2.3.2 Cole and Albicki's Method ......................................................... 24

2.3.3 Zmeureanu's Method ........................................................... 25

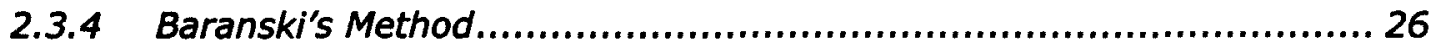

2.3.5 Higher Harmonics Analysis .................................................. 28

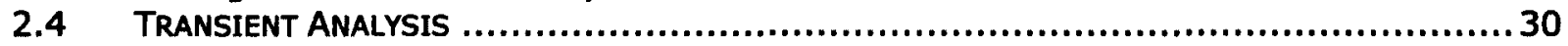

2.4.1 Patel Method ......................................................................... 30

2.4.2 Chang and Lee method ......................................................... 31

3 NON-INTRUSIVE LOAD MONITORING SYSTEM OVERVIEW ............................ 33

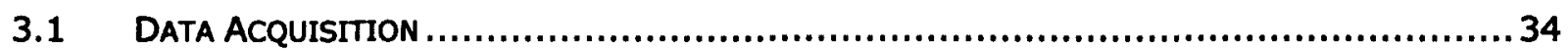

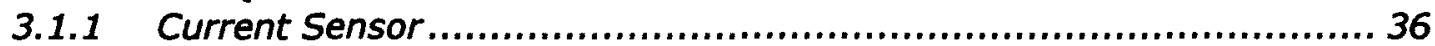

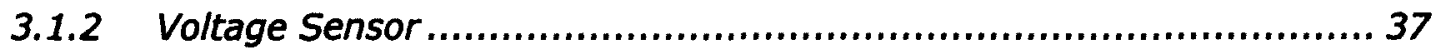

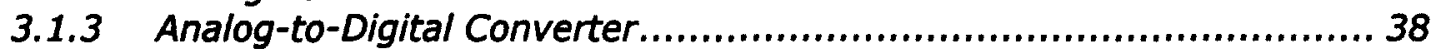

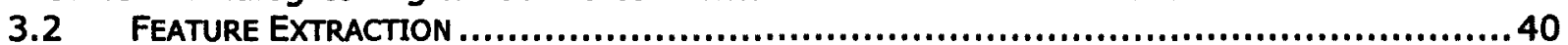

3.2.1 Feature-Additive Criterion .......................................................41

3.2.2 Steady State Load Signature .................................................. 42

3.2.3 Transient Load Signature ...................................................... 47

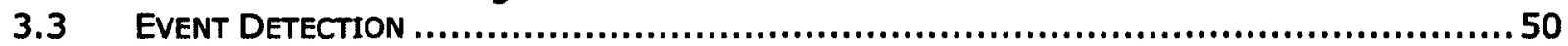

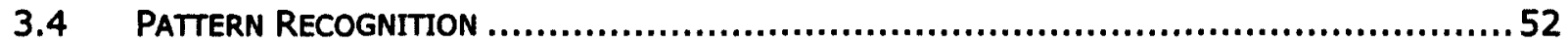


4 STEADY-STATE ANALYSIS............................................................. 55

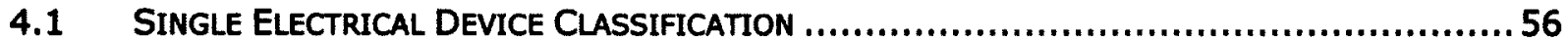

4.1.1 Feature Extraction ........................................................ 57

4.1.2 Pattern Recognition ....................................................... 58

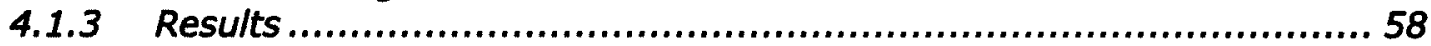

4.1.4 Discussion.................................................................. 60

4.2 CLASSIFICATION OF TWO ELECTRICAL DEVICES OPERATING SIMULTANEOUSLY $\ldots \ldots \ldots \ldots \ldots \ldots \ldots 61$

4.2.1 Data Acquisition............................................................ 62

4.2.2 Feature Extraction ......................................................... 64

4.2.3 Pattern Recognition ..................................................... 65

4.2.4 Results ......................................................................66 66

4.2 .5 Discussion.................................................................. 68

4.3 LOAD IDENTIFICATION DURING THE SIMULTANEOUS OPERATION OF FIVE ELECTRICAL DEVICES. 69

4.3.1 Data Acquisition............................................................. 70

4.3.2 Feature Extraction .......................................................... 72

4.3.3 Pattern Recognition..................................................... 73

4.3.4 Results ..................................................................... 75

4.3.5 Discussion.................................................................... 78

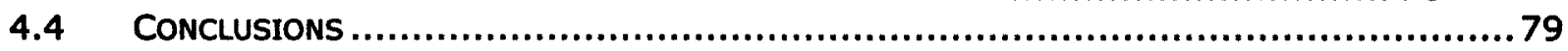

5 TRANSIENT ANALYSIS.............................................................. 80

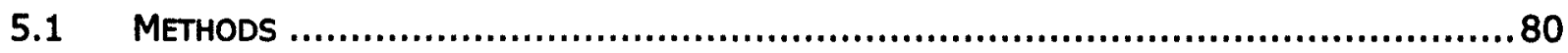

5.1 .1 Data Acquisition............................................................. 84

5.1 .2 Feature Extraction ........................................................ 86

5.1 .3 Event Detection ............................................................ 86

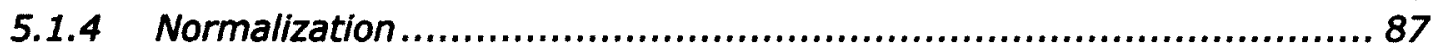

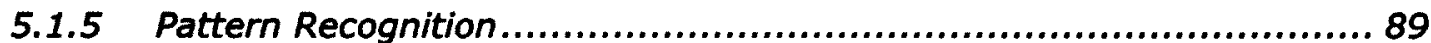

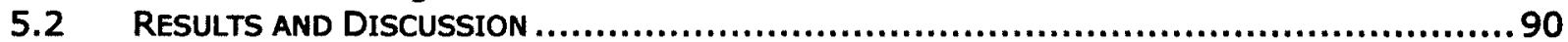

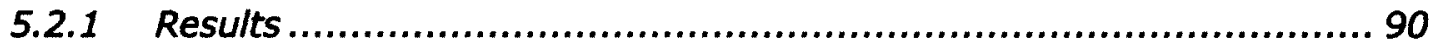

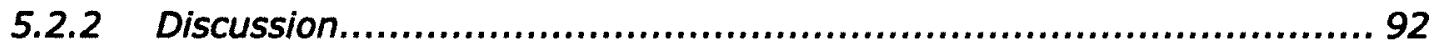

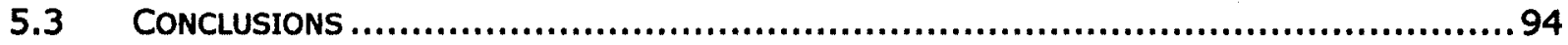

6 THESIS CONCLUSIONS AND FUTURE RECOMMENDATIONS ........................ 95

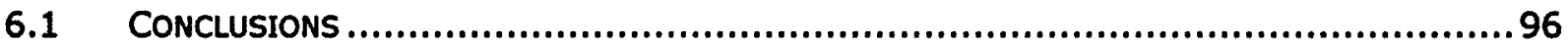

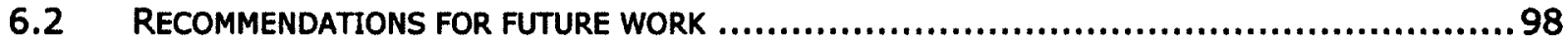

APPENDIX A: DATA AQUISITION DURING THE MULTIPLE OPERATION OF FIVE DIFFERENT ELECTRICAL DEVICES ................................................................. 110

APPENDIX B: SPECIFICATIONS OF ELECTRICAL DEVICES ............................ 113 


\section{List of Tables}

TABLE 4.1: ELECTRICAL DEVICES USED IN EXPERIMENTAL SETUP, ALONG WITH THEIR MEAN REAL POWER AND REACTIVE POWER ( \pm STANDARD DEVIATION), COMPUTED ACROSS ALL ANALYSIS WINDOWS............59

TABLE 4.2: CLASSIFICATION CONFUSION MATRIX FOR REAL POWER (MEAN CLASSIFICATION ACCURACY:

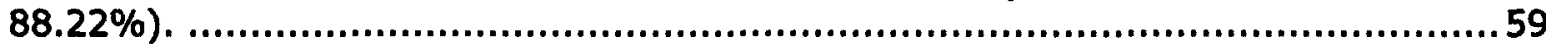

TABLE 4.3: CLASSIFICATION CONFUSION MATRIX FOR REACTIVE POWER (MEAN CLASSIFICATION ACCURACY: $85.79 \%)$. 59

TABLE 4.4: CLASSIFICATION CONFUSION MATRIX FOR REAL POWER AND REACTIVE POWER (MEAN

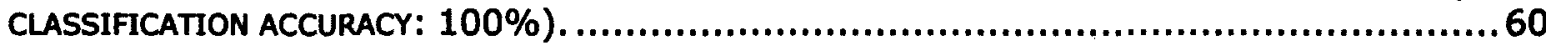

TABLE 4.5: MEAN REAL POWER ( \pm ONE STANDARD DEVIATION) FOR THE ELECTRICAL DEVICES, WHEN

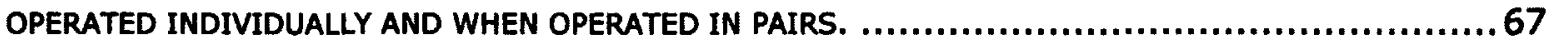

TABLE 4.6: CLASSIFICATION CONFUSION MATRIX FOR REAL POWER (MEAN CLASSIFICATION ACCURACY

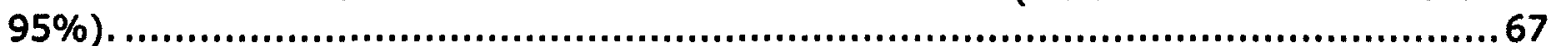

TABLE 4.7: TURNING ON AND TURNING OFF SEQUENCES OF FIVE ELECTRICAL DEVICES DURING EACH MEASUREMENT TRIAL. ......................................................................72

TABLE 4.8: CLASSIFICATION CONFUSION MATRIX FOR IDENTIFICATION OF FIVE ELECTRICAL DEVICES USING REAL POWER USAGE OF DEVICES (MEAN CLASSIFICATION ACCURACY: $86.25 \%$ ). ................. 75

TABLE 4.9: MEAN REAL POWER CHANGE OF 5 ELECTRICAL DEVICES WHEN OPERATING SIMULTANEOUSLY DURING EACH MEASUREMENT TRIAL. THE FIRST AND SECOND VALUES OF EACH CELL REPRESENT THE TURN ON AND TURN OFF REAL POWER CHANGE FOR EACH ELECTRICAL DEVICE. 76

TABLE 5.1: CLASSIFICATION CONFUSION MATRIX FOR LOAD IDENTIFICATION (LOAD 1: KITCHEN BLENDER, LOAD 2: COMPUTER CRT MONITOR, LOAD 3: AIR HEATER, AND LOAD 4: HAIR DRYER) BASED ON THE NORMALIZED SWITCHING ON TRANSIENT WAVEFORMS (MEAN CLASSIFICATION ACCURACY $80.83 \%$ ). 91

TABLE 5.2: LOAD RECOGNITION ACCURACY FOR EACH MEASUREMENT TRIAL AS TRAINING SET...............91 


\section{List of Figures}

FIGURE 2.1: TYPICAL SYSTEM LAYOUT FOR NON-INTRUSIVE MONITORING OF SEVERAL ELECTRICAL DEVICES [BASED ON 28].

FIGURE 2.2: CLASSIFICATION OF LOAD SIGNATURES AND RELATED LOAD IDENTIFICATION METHODS USING

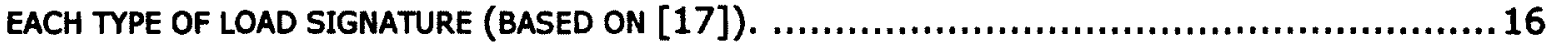

FIGURE 2.3: ACTIVE POWER AND REACTIVE POWER CHANGES DURING ELECTRICAL DEVICES OPERATION.... 18

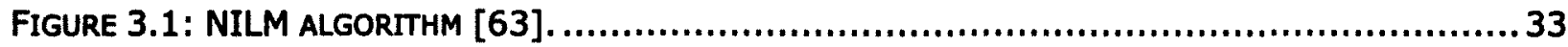

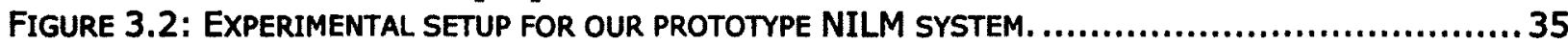

FIGURE 3.3: EXPERIMENTAL HARDWARE USED FOR DATA ACQUISITION AND DATA ANALYSIS............... 36

FIGURE 3.4: VOLTAGE SENSING IN THE NILM EXPERIMENTAL SETUP.................................. 38

FIGURE 3.5: RELATIONSHIP BETWEEN REACTIVE POWER (Q) AND ACTIVE POWER (P) [70].............4 43

FIGURE 3.6: AN EXAMPLE OF SLIDING ANALYSIS WINDOW FOR FEATURE EXTRACTION. REAL POWER VALUES ARE EXTRACTED FROM EACH 100 MS ANALYSIS WINDOWS OF INSTANTANEOUS POWER............45

FIGURE 3.7: EXAMPLES OF A COMPOSITE REAL POWER WAVEFORM (A) AND REACTIVE POWER WAVE FORM (B) FOR MULTIPLE ELECTRICAL DEVICES OPERATION. NUMBERS INDICATE EVENTS (I.E., ELECTRICAL DEVICES TURNING ON OR OFF): (1) COMPUTER LCD MONITOR: ON, (2) FLUORESCENT LAMP: ON, (3) COFFEE MAKER: ON, (4) INCANDESCENT LAMP: ON, (5) ELECTRIC KETTLE: ON, (6) ELECTRIC KETTLE: OFF, (7) INCANDESCENT LAMP: OFF, (8) COFFEE MAKER: OFF, (9) FLUORESCENT LAMP:

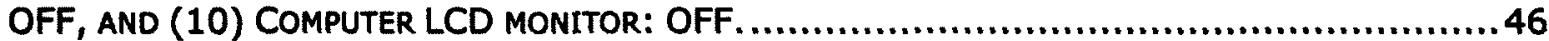

FIGURE 3.8: AN EXAMPLE OF SLIDING ANALYSIS WINDOW FOR STW EXTRACTION (BASED ON [72])......48

FIGURE 3.9: TRANSIENT DATA FOR ELECTRICAL DEVICES SWITCHING FROM OFF TO ON: (A) HAIR DRYER, (B) COMPUTER CRT MONITOR, AND (C) KITCHEN BLENDER...........................................49

FIGURE 3.10: AN EXAMPLE OF EVENT DETECTION ALGORITHM. (A) HEAVY BLACK LINE - ORIGINAL REAL POWER WAVEFORM OF A COMPOSITE LOAD SIGNATURE RELATED TO THE MULTIPLE OPERATIONS OF 5 ELECTRICAL DEVICES IS GIVEN AS AN INPUT TO THE EVENT DETECTION ALGORITHM; (B) DASHED BLACK LINE - DIFFERENCE WAVEFORM IS DERIVED BY USING AN AVERAGING WINDOW TO DETERMINE THE PEAK VALUES; (C) RED CIRCLES - THESE VALUES REPRESENT THE LOCATION OF THE STEP-CHANGE IN THE ORIGINAL AGGREGATE REAL POWER WAVEFORM. ............................................. 51

FIGURE 4.1: EXPERIMENTAL SETUP FOR OUR NILM PROTOTYPE (LOAD IDENTIFICATION DURING THE

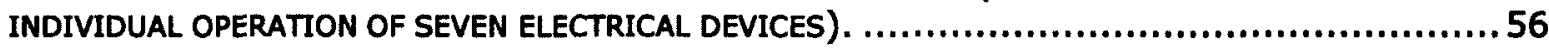

FIGURE 4.2: EXPERIMENTAL SETUP FOR OUR NILM PROTOTYPE (LOAD IDENTIFICATION DURING THE

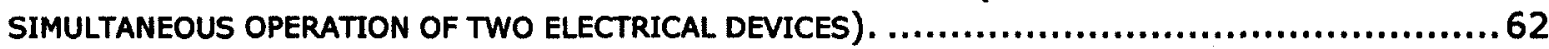

FIGURE 4.3: REAL POWER VERSUS TIME FROM AN EXAMPLE MEASUREMENT TRIAL INVOLVING TWO ELECTRICAL DEVICES: DEVICE 'A' AND DEVICE ' $B$ '. IN THIS PLOT, THE DEVICES WERE ELECTRIC KETTLE AND COFFEE MAKER, RESPECTIVELY........................................................................ 64

FIGURE 4.4: EXPERIMENTAL SETUP FOR OUR NILM PROTOTYPE (LOAD IDENTIFICATION DURING THE SIMULTANEOUS OPERATION OF FIVE ELECTRICAL DEVICES). .................................... 70

FIGURE 4.5: AN EXAMPLE OF REAL POWER WAVEFORM FOR ONE OF THE MEASUREMENT TRIALS FOR THE SIMULTANEOUS OPERATION OF MULTIPLE ELECTRICAL DEVICES. THE CONSEQUENCE OF TURNING ON AND

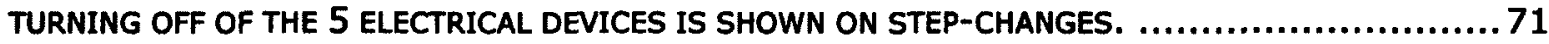

FIGURE 4.6: REAL POWER CONSUMPTION DURING THE MULTIPLE OPERATION OF FIVE ELECTRICAL FOR (A) TRIAL 2 (LOAD IDENTIFICATION ACCURACY 80\%) AND (B) TRIAL 3 (LOAD IDENTIFICATION ACCURACY 100\%). GREEN CIRCLES ARE USED TO INDICATE THE DETECT EVENTS. RED CIRCLES SHOW THE EVENTS IN WHICH LOADS ARE MISCLASSIFIED. ELECTRICAL DEVICES THAT ARE TURNED ON OR OFF ARE INDICATED, WITH THE CLASSIFICATION RESULT IN SQUARE BRACKETS. ...........................77

FIGURE 5.1: EXPERIMENTAL SETUP FOR OUR PROTOTYPE NILM SYSTEM FOR CLASSIFICATION OF SINGLE ELECTRICAL DEVICES BASED ON THEIR TRANSIENT PROFILES. 81 
FIGURE 5.2: SWITCHING ON TRANSIENT PROFILE OF THREE DIFFERENT ELECTRICAL DEVICES (REAL POWER VALUES VERSUS TIME): (A) COMPUTER CRT MONITOR, (B) KITCHEN BLENDER, AND (C) HAIR DRYER. 83

FigURE 5.3: (A) RMS CURRENT VERSUS TIME, (B) RMS VOLTAGE VERSUS TIME, AND (C) REAL POWER VERSUS TIME FROM AN EXAMPLE MEASUREMENT TRIAL FOR LOAD 2. 85

FIGURE 5.4: AN EXAMPLE OF SLIDING ANALYSIS WINDOW FOR FEATURE EXTRACTION (BASED ON [72]). .. 86

FIGURE 5.5: REAL POWER VERSUS TIME FROM AN EXAMPLE MEASUREMENT TRIAL SHOWING THE SWITCHING ON/OFF TRANSIENT WAVEFORM OF LOAD 2 (COMPUTER CRT MONITOR). GREEN CIRCLES SHOW THE SWITCH ON/OFF EVENTS; THE FIRST SEGMENT (RED AREA) SHOWN AS SEGMENT A IS NORMALIZED AND USED AS SWITCHING ON TRANSIENT WAVEFORM WHILE THE SEGMENT B IS USED TO CALCULATE THE STEADY-STATE REAL POWER VALUE. 88

FIGURE 5.6: SWITCHING ON TRANSIENT WAVEFORMS (MEASURED NORMALIZED REAL POWER) DURING 10 DIFFERENT MEASUREMENT TRIALS FOR EACH INDIVIDUAL ELECTRICAL DEVICE: (A) KITCHEN BLENDER, (B) COMPUTER CRT MONITOR, (C) AIR HEATER AND, (D) HAIR DRYER.

90

FIGURE 5.7: NORMALIZED REAL POWER VERSUS TIME DURING 10 DIFFERENT MEASUREMENT TRIALS TO RECORD THE SWITCHING ON TRANSIENT WAVEFORM OF LOAD 2. THE BLACK DASHED LINE INDICATES THE TRANSIENT BEHAVIOUR OF LOAD 2 DURING MEASUREMENT TRIAL 9. THREE DIFFERENT AREAS ARE MAXIMIZED TO SHOW THE DIFFERENCE BETWEEN THE SWITCHING ON TRANSIENT WAVEFORM OF LOAD 2

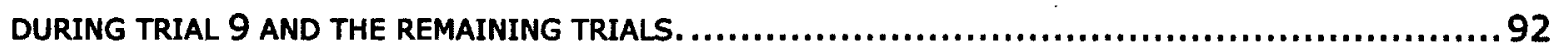




\section{List of Abbreviations}

\begin{tabular}{|l|l|}
\hline ADL & Activities of Daily Living \\
\hline ANN & Artificial Neural Networks \\
\hline CRT & Cathode Ray Tube \\
\hline CSCW & Computer Supported Cooperative Work \\
\hline CW & Current Waveform \\
\hline DAQ & Data Acquisition Device \\
\hline EMI & Electromagnetic Interference \\
\hline FFT & Fast Fourier Transform \\
\hline IAW & Instantaneous Admittance Waveform \\
\hline ILM & Intrusive Load Monitoring \\
\hline IPW & Instantaneous Power Waveform \\
\hline KNN & k-nearest neighbor \\
\hline LCD & Liquid Crystal Display \\
\hline NI & National Instruments \\
\hline NILM & Non-Intrusive Load Monitoring \\
\hline RFID & Radio Frequency Identification \\
\hline RMS & Root Mean Square \\
\hline SMPS & Switch Mode Power Supplies \\
\hline STW & Switching Transient Waveform \\
\hline SVM & Support Vector Machine \\
\hline THD & Total Harmonics Distortion \\
\hline VSD & Variable Speed Drivers \\
\hline
\end{tabular}




\section{Introduction}

\subsection{Overview}

\subsubsection{Introduction to Smart Homes}

Developed countries are facing a shift in their demographics with an aging population growth. United Nations estimates that by the year 2050, nearly 1 in every 6 persons throughout the world is projected to be at least 65 years old [1]. The average age of the population is significantly growing in Canada; for the year 2021, Health Canada predicts $18 \%$ of population (6.7 million Canadians) over 65 [2]. The increasing proportion of older adults makes it an essential task for today's societies to improve the daily living standards for the ageing population. Meanwhile, the current healthcare system is inadequate for a larger older demographic. Hospitals and long 
term care facilities cannot support this large number of people [3][4]. Simultaneously, older adults prefer to live in their own home as long as possible. Therefore countries have to be prepared to develop technologies to mitigate the pressures on the health care system from the aging population by supporting "aging in place" [5]. The concept of ageing in place aims to help elderly people live at homes instead of going to hospitals or long term care facilities.

The ageing process is associated with some harmful risks in the daily life of older adults (like falls) and some age related diseases (such as cognitive impairments, Dementia and Alzheimer) which makes it difficult for them to be able to live alone independently [5][6]. As a result, hospitalization in costly clinical institutions becomes necessary where seniors can receive stable therapeutic supervision [6]. Unfortunately, this solution is not always possible because of shortage of services by overcrowded existing hospitals and nursing care facilities [6]. Therefore, there is a growing demand for alternative solutions to help elderly people to live in their private homes as long as possible [7][8]. This provides the impetus of the smart home concept. The main goal of smart home developers is to identify and present a home-based automated system, which monitors the elderly people's health and wellbeing, while remaining unobtrusive and provides them with various facilities such as more comfortable lifestyle, higher degree of 
independence (self living environment), and more affordable care (also decreasing the financial burden on the healthcare system) [7].

The objective of smart home technologies is to develop an "Independent Living Home" by monitoring the Activities of Daily Living (ADL) of elderly people [7]. A smart home system can be used to detect abnormal activities and changes in behavioral patterns, which may be indicative of an underlying health and wellness issue. For instance, suppose that an electrical device such as an stove has been ON for long period of time; monitoring the usage of this electrical device can help us to predict an abnormal behaviour of occupants (Increasing and regular burning of food, due to forgetting to turn off the stove can be indicative of dementia)._According to the detected abnormal behaviours, decisions will be made regarding the resident's status and physiological condition in order to provide them with the appropriate care and emergency aid. The smart home can provide notification for emergency cases such as burning stove or a hard fall, subsequently providing the appropriate assistance [7].

Available smart home technologies, though based on different techniques, have several common principles. First, specific intelligent sensors need to be designed in order to monitor ADLs of residents in a smart home. Second, data acquisition and processing are required to analyze suitable data from all sensors throughout the smart home. Last, an expert system correlates information from all of the sensors throughout the home 
and makes decisions regarding the resident's status and physiological condition [7]. Many different types of intelligent sensors such as magnetic switches, thermistors, accelerometers, infrared motion sensors, microphone arrays, smart grab bars, pressure sensitive mats, electronic noses, Radio Frequency Identification (RFID) networks and video cameras have been investigated in several previous researches [7][9][10][11][12]. In this thesis we are researching methods to provide the usage profile of electrical devices (load monitoring) to support ADL monitoring. Load monitoring techniques would be one of many different types of intelligent sensors as mentioned above in smart homes. Information from other sensor systems throughout the smart home can resolve issues such as discerning which person is operating the electrical devices (if there is more than one occupant in the smart home) and discerning similar electrical devices in different rooms (e.g., same light bulb in different rooms).

\subsubsection{Load Monitoring Concept}

Load monitoring systems are expected to play a key role in smart homes; they can provide information about the operating schedule [13] of electrical devices (when electrical devices e.g., stove, microwave, lights are in use). Pioneering works in load monitoring were mainly done to reduce energy consumption of different groups of consumers by providing a detailed consumption report. There were also other areas of interest in load 
monitoring such as collecting information about electric energy consumption patterns and monitoring the state of electrical devices for fault diagnostics [14][15]. In this research we are looking at ADL monitoring of ageing population. In the attempt of monitoring the ADLs of elderly people, we aim to identify the operating schedule of each individual electrical device. The operating schedule for each device includes the time when each device is turned ON/OFF and the duration of operation.

Load Monitoring approach in this work aims to detect a variety of electrical events throughout the smart home. ADL of elderly people in smart home can be predicted by observing actuation of certain electrical devices [5]. For example, detecting that a light switch was turned on can be an indication that someone entered a room. Other $A D L$, such as turning on a microwave or kitchen blender may indicate meal preparation [16]. Activity sensing in the smart home by load monitoring systems has a variety of important applications, including health care, home automation, and energy monitoring.

\subsubsection{Types of Load Monitoring Techniques}

Load monitoring techniques at the household level can be broadly classified into two classes: Intrusive Load Monitoring (ILM) systems and Non-Intrusive Load Monitoring (NILM) systems [17][18][19]. 


\section{Intrusive Load Monitoring}

The most important step to monitor the ADL through load monitoring is determining the operating schedule of each electrical device within a smart house. The easiest way of detecting the usage of electrical devices is achieved by installing sensors for each load. The status of the load is transmitted to the main controller such that the status of each load can be monitored. The sensors send the power usage information to the control device in order to keep track the usage of each device [20]. However, this method requires a large amount of sensors. The operation schedule of electrical devices are determined more precisely by using Intrusive Load Monitoring (ILM) systems (refers to standard sub-metering techniques) which are able to provide highly accurate data by employing a network of sensors or meters attached to each individual electrical device $[17][21][22][23]$. However, the sub-metered data is achieved at the expense of expensive installation hardware, complexity of system and customer discomfort [17][24][25][26]. Meanwhile, the appearance of many sensors attached to electrical devices detracts the aesthetics of the home [5].

\section{Non-Intrusive Load Monitoring}

Non-intrusive methods are intended to offer installation simplicity and the ability to distinguish individual loads via measurement at a central 
monitoring point [17][22]. The nonintrusive load monitoring (NILM) method requires a set of current and voltage sensors that are installed at the main electrical panel [20]. By analyzing the current and voltage waveforms, the power usage of each load can be detected. Compared to ILM, the NILM has several advantages, e.g., easier to install, lower cost, lower energy consumption, etc [20]. In this research, we are looking at NILM systems; a principle advantage of these systems is that it requires only the installation a single sensor placed in the main electrical entrance board of a smart home.

\subsection{Objectives}

The overall objective for this research is the development of a non-intrusive load monitoring system for smart home purposes, with the underlying goal of predicting the ADL of elderly people. In this thesis, our objective is to investigate the feasibility of proposed methods and algorithms in a procedure for monitoring multiple electrical devices. It is focused upon the accepted techniques for NILM systems using steady-state features (i.e., real power and reactive power) and transient features (i.e., switching on transient waveforms) as reliable indicators for various electrical devices recognition. The execution of load identification is demonstrated by implementing a prototype NILM system and testing this system on multiple common household electrical devices. 


\subsection{Thesis Scope}

In this work, electrical devices with more than one electrical load (e.g., a clothes dryer contains a heating element and a motor - each component representing a different load) and continuously varying devices were not considered. Data were acquired from two-state and multi-state electrical devices. Multi-state electrical devices were considered in only one of their operation states (I.e., one of their ON states). For instance, data from a microwave was acquired from only one operation state of this device (reheat). In this work, multiple electrical devices turning on/off simultaneously were not explored; we consider cases where only one electrical device changes state (ON/OFF) within a small time interval. It is been expected that the possibility of two electrical devices turning on/off at the same time is small.

\subsection{Contributions}

The following is a list of the three main contributions of this work with brief descriptions of each:

1. Implementation, validation, and testing a prototype NILM system for usage monitoring of electrical devices: In this thesis, a prototype NILM system was developed to conduct validation experiments with various common household electrical devices. 
Experiments showed that the operating schedule of electrical devices can be determined by using steady-state features (i.e., real power and reactive power) or transient features (i.e., switching on transient waveforms), for describing the load signatures of individual electrical devices. This prototype NILM system can be used in on-going researches.

\section{The identification of switching on transient waveforms as a} unique feature for electrical device recognition: This work is not the first study to consider transient behavior of electrical devices, however, to the knowledge of the author this work represents the first study to consider the shape of switching on transient waveforms of electrical devices, for device recognition. In the previous works, transient features such as turn-on transient energy or transient behavior length were used for load identification. In this work, correlation between switching on transient shape of several electrical devices was used to identify the usage of electrical devices based on their transient behavior. Transient analysis experiments conducted with five different electrical devices confirmed that electrical devices can be distinguished based on their switching on transient waveforms. 


\section{The usage of a simple classification method for pattern}

recognition: Many researchers have tried different classification methods such as fuzzy logic, dynamic time warping, back propagation artificial neural networks, cluster matching, and support vector machine in NILM systems for load identification. Since, using these pattern classification methods make the NILM system more complicated and increase the cost and implementation difficulty, we have used a simple classifier method. In this work, pattern classification was performed using discriminant analysis using Mahalanobis distances or a k-nearest neighbor (KNN) classifier $(K=1)$ which are less susceptible to overtraining. With a known electrical device database and the extracted features, these classification methods identify which electrical device has been switched on/off and when (at which time). Although the classification methods used in this work are simple, experimental results show that high electrical device recognition accuracy can be achieved.

These contributions were disseminated in:

- S. Rahimi, A. D. C. Chan, R. Goubran, "Usage monitoring of electrical devices in a smart home", accepted to 32nd Annual International Conference of the IEEE-EMBS, Boston MA, USA, 2011.

- S. Rahimi, A. D. C. Chan, R. Goubran, "Nonintrusive Load Monitoring of Electrical Devices in Health Smart Homes", submitted to IEEE International Instrumentation and Measurement Technology Conference, Graz, Austria, 2012. 


\subsection{Organization of Thesis}

This thesis consists of 6 chapters. The remainder of this dissertation is organized as follows. Chapter 2 presents background and review of the literature in the field of non-intrusive load monitoring systems. Chapter 3 describes an overview of a prototype NILM system presented in this work, completely. Chapter 4 describes our steady-state based methodology for the NILM systems including data acquisition, feature extraction, pattern recognition, results, and discussion. In Chapter 5, our methodology for the NILM systems based on the switching transient behaviour of electrical devices is presented. Chapter 6 summarizes the research conclusions and discusses possible improvements and directions for future work. 


\section{Background and Literature Review}

This chapter provides a high level overview of Non-Intrusive Load Monitoring (NILM) systems and current literature on the topic. Section 2.1 describes the basic principles behind NILM systems, where they are used to identify the operating schedule of individual electrical devices. Central to NILM's is the concept of a load signature (Section 2.2), which provides information about the operating state of an individual electrical device in the total load [27][. For monitoring systems, it is possible to analyze data in steady-state and in the transient state. A literature review on the state of the art on NILM methods is performed for both steady-state methods (Section 2.3) and transient analysis methods (Section 2.4). 


\subsection{NILM Systems}

The NILM is a way to determine the operating schedule of individual electrical devices on an electrical service from measurements of the combined power usage of multiple electrical devices [27]. Non-intrusive methods provide the ability of usage monitoring of all connected electrical devices via measurements from one central point (e.g. main electric utility service entry) as depicted in Figure 2.1 [29]. This type of load monitoring is referred as non-intrusive because it allows tracking the usage of electrical devices without placing individual sensors into each individual electrical device. In order to decompose the power usage of the total load to its components, we need a load disaggregation method. Various load disaggregation techniques are applied to NILM systems in order to distinguish between different electrical devices for specific monitoring of each individual electrical device [30].

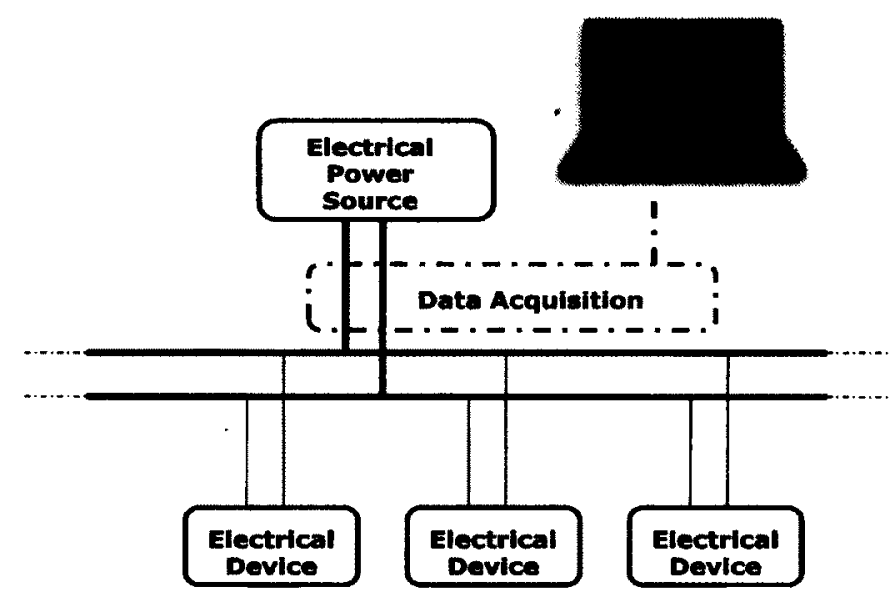

Figure 2.1: Typical System layout for non-intrusive monitoring of several electrical devices [based on 28]. 
The process of load monitoring by NILM systems can be broken down into three main steps [23]: feature extraction, event detection, and pattern recognition. In the feature extraction step, current and voltage waveforms are captured and features such as real power, reactive, power, and harmonics, are extracted from the waveforms. Changes in the features are flagged as events during the event detection step, which indicates devices powering on/off or changing states. Pattern recognition uses a trained classifier to map events to electrical devices and their operation state (e.g., ON or OFF).

Generally, the performance of NILM systems is affected by the following factors: (1) sampling frequency, (2) resolution of analog to digital convertor, (3) power measuring range, (4) electrical noise in the system, and (5) the quantity of individual electrical devices being monitored [17][22]. More advanced NILM systems have been introduced over the years to recognize a variety of electrical devices in residential and commercial buildings. NILM systems have evolved through the improvements in their hardware installations, such as higher sampling frequencies to analyze transient behavior of electrical devices and improved disaggregation algorithms [30].

\subsection{Load Signature}

At the core of load monitoring approaches is the need to understand electrical behavior of devices [31][32]. NILM systems identify individual 
electrical devices based on their load signatures [27][33][34][35]; the term load signature is defined as the electrical behavior of an electrical device during operation, which differs from device to device. Each electrical device contains unique characteristics in its consumption behavior similar to any human's signature (electrical device fingerprint) [27]. Parameters which can be used as load signature includes current, voltage, power measurements, harmonics and switching on/off transient signal.

In NILM systems, what is measured at the main electric entrance (utility service) is composite load signature; the composite load signal refers to the net behavior of more than one electrical device operating simultaneously [27]. A load disaggregation method is used to discern individual electrical devices within the composite load signal. The composite load signature is generally the easiest accessible and less unobtrusive signal; when we monitor composite load signature from a single monitoring point there is no need to instrument every electrical outlet or every electrical device with monitoring hardware. The disadvantage is that it is complicated in nature because certain features from each individual electrical device can be masked or lost [27].

In general, two different types of load signatures, steady-state and transient-state signatures, are used in the field of NILM for load identification purposes [28] (Figure 2.2). The monitoring and recording capability of NILM 
systems determines the choice of a particular load identification method [17].

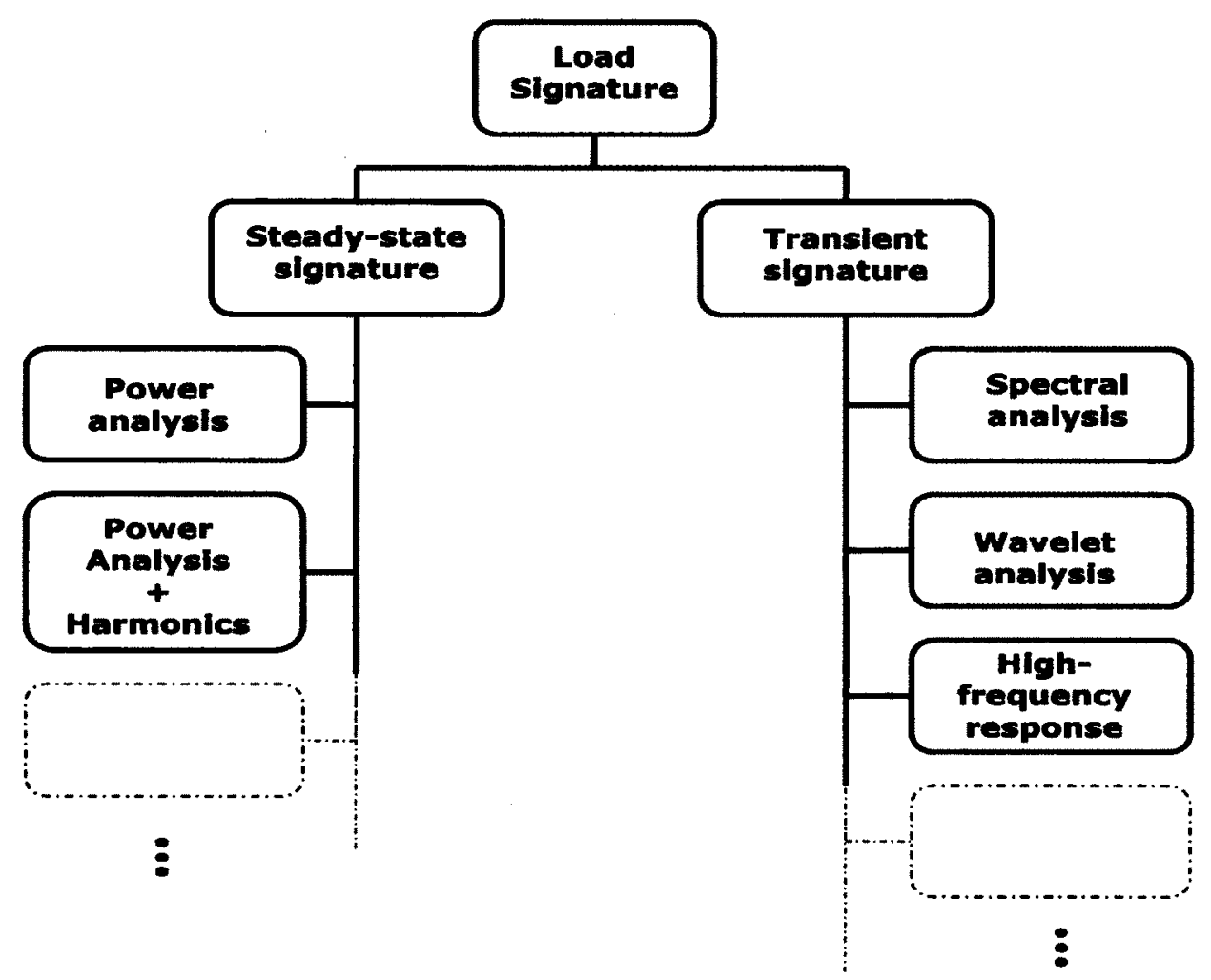

Figure 2.2: Classification of load signatures and related load identification methods using each type of load signature (based on [17]).

\subsubsection{Steady-State Signature}

Steady-state analysis looks at the long-term step changes in power signatures (such as real power and reactive power waveforms) that occur when a particular electrical device turns off or on [29].

\section{A. Power Analysis}

In the power analysis, the operating schedule of an individual electrical device is determined by identifying times at which its active and/or reactive 
power consumption measurements change from one nearly constant (steady-state) value to another [28][36]. Changes known as events, are related to electrical devices either turning on or off. These steady-state changes are characterized by the magnitude and sign of changes in real power $(\Delta \mathrm{P})$ and/or changes in reactive power $(\Delta \mathrm{Q})[36]$. The characterized events are located in a two dimensional (2D) signature space of real and reactive power ( $\Delta \mathrm{P}-\Delta \mathrm{Q}$ plane). This plane reduces the complicated load signatures to a 2D signature space changes in power consumption. Recorded turn on and turn off events are used for matching the positive and negative clusters of similar magnitudes of the measured features [36]. The main limitation of this method is its inability to identify linear and non-linear loads that overlap ambiguously in the $\Delta \mathrm{P}-\Delta \mathrm{Q}$ plane [25].

Three key assumptions have been identified that limit the effectiveness of steady-state analysis [36]:

1. The first assumption is that various electrical devices are identified based on their signatures on two-dimensional signature-space of $\Delta \mathrm{P}$ $\Delta \mathrm{Q}$ plane. The limitation is that the electrical devices with the similar real power and reactive power will be indistinguishable on the $\Delta \mathrm{P}-\Delta \mathrm{Q}$ plane.

2. The second limitation is that using only steady-state signatures for load composition requires waiting until transient signals settles out, then, steady-state values will be measured. 
3. The third limitation is that most steady-state based NILM systems need training period processing data in batch format using a day or more to collect electrical behaviour of devices. This limits near realtime load identification.

An example plot, shown in Figure 2.3, illustrates the steady-state real power and reactive power changes of a composite load consisting of two electrical devices ' $A$ ' and ' $B$ '. In the example, electrical device ' $A$ ' is initially ON, then, electrical device ' $B$ ' is turned on and later electrical device ' $A$ ' is turned off.
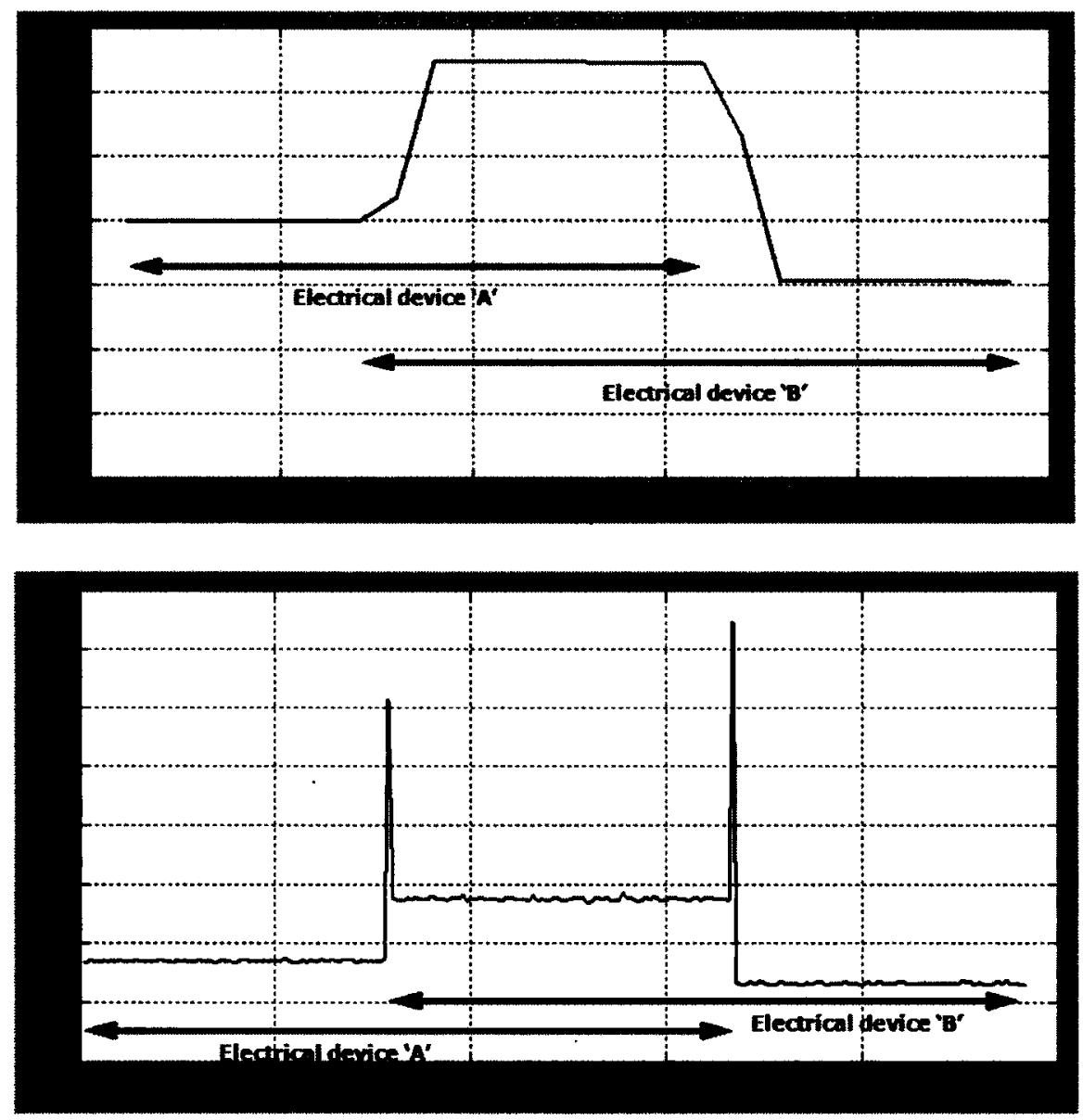

Figure 2.3: Active power and reactive power changes during electrical devices operation. 


\section{B. Harmonic analysis}

Harmonics are used as complementary features, in addition to the changes of real and reactive power; it is suitable for identification of non-linear loads and especially in case of overlapping in $\Delta \mathrm{P}-\Delta \mathrm{Q}$ plane [17][28][36]. A set of higher harmonics can be obtained by a Fast Fourier Transform (FFT) of the aggregate current signal [28][37][38]. By using higher harmonic current and variations in power signatures $(\Delta \mathrm{P}$ and $\Delta \mathrm{Q})$, easier identification can be achieved specially for electrical devices with large harmonic contents [17]. However, harmonic analysis is still incomplete to identify highly resistive loads because their harmonic content is very small. Since this method still requires steady-state measurement it cannot identify continuously variable loads [17][28].

\subsubsection{Transient Signature}

Major electrical devices can be recognized based on their transient signatures. The transient behavior of an electrical device (during turn on and turn off) is a reliable indicator for load identification [28][41][42]. The shape of the transient signatures corresponds to the electrical behavior of certain class of loads and is related to the physical task of the electrical devices [36][40]. Transient signatures exhibit variability in parameters like duration, shape and size, because transient measurements depend on the exact point in the voltage cycle at which the turn on or turn off occurs [17][39]. 
Transient signatures are useful to identify electrical devices that exhibit similar steady-state signatures (but with unique transient turn on characteristics) and also in situations where rapid activation of loads is common such as in commercial and industrial facilities [17][36]. Major limitations of transient analysis are the need for high sampling frequency and relatively unique and reasonably repeatable transient patterns (Transient signatures may not be repeatable for all state changes of an individual electrical device) [28].

\subsection{Steady-State Analysis}

\subsubsection{Hart Method}

The concept of nonintrusive load monitoring (NILM) was first presented by George Hart in the 1980's at MIT [36]. Hart patented a method for determining the operating schedule of individual electrical devices within a residential building [28]. The presented NILM system by Hart [43] estimated the number and nature of individual loads by analyzing current and voltage waveforms obtained at the main feed and sampled at $2 \mathrm{kHz}$ (Hertz), using normalized real power and reactive power measurements. Computation of active and reactive power was done three times per second [43][44].

Under Hart's method, load disaggregation process starts with detecting the times at which power measurements change from one nearly constant 
steady-state value to another known as events. These steady-state changes (events) correspond to the load either turning on or turning off and are characterized by their magnitude and sign in real and reactive power. Then, detected events with equal magnitudes and opposite signs are paired to constitute a library of operating cycles and the power consumption of each load. Eventually, a trained algorithm that matches the sample feature with known events (previously recorded for each individual load) is used for load disaggregation purposes [36][43].

The established version of the MIT method describes a five step load disaggregation process through the detection of changes in aggregate power consumption, as following [43][44][45]:

1. Event detection: the process begins by detection of the events (stepchanges) which separates one steady-state from another in order to determine changes in steady-state levels.

2. Cluster analysis: in this step, similar sets of events are grouped together based on their magnitudes giving positive clusters (turn on) and negative clusters (turn off). This algorithm is used to locate the steady-state changes in a $\Delta \mathrm{P}-\Delta \mathrm{Q}$ plane. The positive and negative clusters are quite distinct for major electrical devices; however, there is probability of overlapping clusters in $\Delta \mathrm{P}-\Delta \mathrm{Q}$ plane.

3. Cluster matching: each positive cluster is matched or paired with a similar negative cluster based on their magnitude. For instance, 'two- 
state' loads consume a relatively constant power during their turn on and turn off.

4. Anomaly resolution: unmatched clusters that may arise from situations such as missed events or simultaneous activation of loads are paired or associated with existing or new clusters according to a best likelihood algorithm.

5. Appliance identification: the matched clusters are associated with known power consumption of electrical devices; the comparison is based on power signatures. This step uses information collected during a training procedure (which is actually an intrusive, thought one-time procedure) or using historical data.

This five-step approach has been successful in determining the operating schedule of certain classes of electrical devices. The MIT group considered three classes of electrical device models for the NILM approaches [43][45]:

ON/OFF electrical devices: Most household electrical devices, such as a toaster, light bulb, or water pump belong to this category. According to the Boolean switch function the ON/OFF model electrical device can only be either ON or OFF at any given time. Electrical devices with distinct types of ON states such as a typical toaster oven (bake/broil/toast), three-way lamp (low/medium/high), or washing machine (fill, agitate, spin) cannot be classified in this group. 
Finite State Machine (FSM) appliances: The finite state machine (FSM) model allows for an arbitrary set of discrete states and state transitions. This category includes electrical devices which pass through several definite switching states (electrical devices with more than one distinct on power state). Examples of FSM are a washing machine, fan, or clothes dryer.

Continuously Variable loads: The third classes of electrical device models, continuously variable appliances have an infinite number of states. Continuously varying electrical devices such as light dimmers, sewing machines, and variable speed drills, do not consume a constant power, and so do not generate consistent step-change signatures.

The MIT group reported that their method (5-step load disaggregation process) is applicable to only the ON/OFF electrical devices [43]. This category of electrical devices could be detected and monitored using the changes of real and reactive power as the features. Later, in [46] and [47] the MIT group proposed various methods for detection and monitoring of FSM (second category of appliances) and continuously varying loads.

It is reported that the 5-step NILM method by MIT group is not able to detect small loads due to the higher setting of event threshold [17]. Furthermore, permanent consumer devices which are in continuous operation all the time cannot be detected using this method; consumer devices remain on for 24 hours a day, 7 days a week, with approximately 
constant active and reactive power draw. Examples of devices in this category include hard-wired smoke alarms and some external power supplies [44]. Also, the electrical devices consuming similar power (e.g., a computer and an incandescent bulb) may not be separated by this method [36][44]. Lastly, the matching algorithm of this method is based on the assumption that the positive change of power (turn on) matches the negative change of power (turn off). However, the impedance load of many electrical devices change over time which results in changes in their power usage; a mismatch can occur due to this power drift [32].

\subsubsection{Cole and Albicki's Method}

Cole and Albicki (1998) developed a modified version of Hart algorithm which recognizes at least 6 major residential electrical devices including Air conditioner, Electric clothing dryer, Heat pump, Clothes washer, Water heater and Refrigerators [44][48]. Cole and Albicki's method uses sampled data for real power and reactive power to identify electrical devices with significant spikes in their power draw [44]. The algorithm detects the usage of these major electrical devices when the physical components of the electrical devices turn on and off. Moreover, the algorithm incorporates a logical analysis to separate between simultaneous ON/OFF events, when the ON-OFF times of more than one electrical device overlap. This method is not able to detect any electrical device power usage that does not change [48]. 
Load identification by Cole and Albicki's method is based on the use of 'edges' and 'slopes' as features of the steady-state power draw of electrical devices. Edges are defined as the abrupt changes in power level, while, slopes are defined as the slower changing variation that occurs during the turn-on events. For load identification, current and voltage waveforms were sampled at the meter and processed into real and reactive power data. Lastly edges and slopes were identified for load disaggregation [49][50].

\subsubsection{Zmeureanu's Method}

Farinaccio and Zmeureanu (1999 - 2000) developed a NILM approach by using the changes in real power data (sampling rate: $16 \mathrm{~s}$ ) along with electrical device-specific decision rules [19][44]. Their method shows a promising potential for NILM application in residential buildings to reliably detect major end-uses such as Refrigerators and Water heaters, using a pattern recognition approach. Moreover, this method required a training period of about a week in order to find the load characteristics of the targeted individual electrical devices. Furthermore, this method relies on complicated decision rules; for each individual electrical device a nonintuitive decision rule is defined. For example, a decision rule for Water heater is based on the normalized start or end demand profile respect to the average normalized start or end demand profile from the training data which is different than the defined decision rule for refrigerator [21]. The need for 
proposing decision rules specific to each individual electrical device is the limitation of this work [44].

This research group improved their method in [51] by using changes in real power data along with signal filtering/smoothing and stopping the use of arbitrary electrical device-specific decision rules (pattern recognition rules of their previous work [19]) to detect ON/OFF events. Instead, during the training period the usage duration of targeted electrical devices were accumulated in order to detect electrical devices based on their usage duration profiles [51]. Therefore, the new method was able to distinguish between two different electrical devices with similar real power but different usage durations.

The new extended method was able to detect the usage of seven major household electrical devices with the load identification accuracy of up to $90 \%$ including Water heater, Refrigerator, Clothes washer, Stove, Clothes dryer, Dish washer, and Baseboard heater [51]. The limitation of this work similarly to many of NILM techniques is the need for excessive training period (about a week) to record the usage profile of electrical devices of interest [44].

\subsubsection{Baranski's Method}

Baranski and Voss [52][53][54] presented a complex NILM algorithm combining clustering, genetic algorithms, finite-state machines and 
heuristics. Power data were acquired from an optical sensor installed on the utility's meter, greatly reducing the cost of implementation [44]. Baranski and Voss presented a unique NILM approach [52] to analyze data, collected from a particular measurement system. The measurement system was based on a conventional power meter. The power meter was complemented by a low cost optical sensor that continuously monitored the overall real power (sampling frequency: $1 \mathrm{~Hz}$ ) without any intrusion into the main electrical installation of the household (the optical sensor was used to visually read the conventional power meter's display) [53].

In order to decompose the aggregate real power signal to its components which are major electrical end uses, a load disaggregation algorithm consisted of fuzzy clustering method, a genetic algorithm and a dynamic programming approach was used. The implemented clustering method was used to finds typical switch states of frequently occurring electrical devices. Moreover, a genetic algorithm was used to recognize finite state machines due to constraints of electrical end uses. Then, fuzzy sets were used in order to solve the problem of overlapping of multiple assigned switch events. Ultimately, this method was able to recognize typical patterns of ON/OFF electrical devices and finite state machines (with less than about five different states) [53]. Unlike most other NILM techniques, training period was not required in this work. Instead, for load identification detecting switch events in consideration of load peaks were the only information this 
system used. The limitation of this work is the proposed genetic algorithm as an optimization algorithm; this algorithm is a simulation method that may or may not provide the optimal solution [44].

\subsubsection{Higher Harmonics Analysis}

The NILM approach developed in [36] relies on higher harmonic analysis. In this method, presented by Laughman et al., short-time Fourier transform of current waveforms collected at a sampling frequency of $8000 \mathrm{~Hz}$ is used to compute harmonic contents (up to $7^{\text {th }}$ harmonics) [17]. The short-time Fourier transform computes real power, reactive power, and higher frequency components of the current. Higher harmonics can be used to distinguish electrical devices with overlapping clusters in the $2 \mathrm{D} \Delta \mathrm{P}-\Delta \mathrm{Q}$ plane [28].

The advantage of harmonic analysis was elaborated by a load disaggregation example in [36]. Linear Incandescent Lamp and Computer Power Supply are practically indistinguishable in the $\Delta \mathrm{P}-\Delta \mathrm{Q}$ signature space because both consume relatively similar real and reactive power. However, these two electrical devices are distinguishable by examining higher harmonics [17][36][44].

The authors of [36] found out that the third harmonic which is characteristic to each individual electrical device can be used for better discrimination between these two electrical devices. Figures 6 and 7 from 
[36] illustrate the separation between a computer and an incandescent lamp in a three-dimensional space with axes denoting changes in real power, reactive power, and third harmonic.

The MIT group led by S. Leeb and L. Nford extended their harmonic analysis in [40] and [58] by introducing the concept of 'Spectral Envelope'; which is a vector of the first several coefficients of the short-time FFT of the signal [44]. The spectral envelopes are characteristic to each individual electrical device and can be. used as unique features for better identification of continuously varying loads (third class of electrical devices) [40]. In a more recent work by this group in [56] and [57], correlations between $5^{\text {th }}$ and $7^{\text {th }}$ harmonics along with the changes in real power and reactive power were used to recognize a Supply Fan in a large building [44].

Harmonic analysis presented in this work suffers from several limitations. Firstly, an excessive training period to profile the characteristics of targeted electrical devices. Secondly, the electrical device recognition accuracy has not been characterized for practical situations. Lastly, it is not known how the presence of new electrical devices affects the NILM performance [44]. 


\subsection{Transient Analysis}

\subsubsection{Patel Method}

S. Patel et al. in [16] have presented a new NILM approach that uses a single plug-in sensor (that is plugged into any electrical outlet in the home) to detect the operating schedule of various electrical devices. A personal computer is used to record and analyze electrical noise on the power line caused by the switching on/off of the electrical devices. This system learns certain characteristics from transient signatures produced by switching an electrical device on or off [16]. A training period is required for this system to learn certain characteristics from electrical noise produced by switching an electrical device on or off. Based on the learned phenomena, machine learning techniques (Support Vector Machine - SVM) are employed in order to identify when unique events occur. Results of this work showed that the presented method can learn and classify various electrical events with accuracies ranging from $85-90 \%[16][44]$.

S. Patel et al. presented a new solution in [59], for NILM approaches in their more recent work. ElectriSense relies on the fact that most modern consumer electronics and fluorescent lighting employ switch mode power supplies (SMPS) to achieve high efficiency that continuously generate high frequency electromagnetic interference (EMI) during operation that propagates throughout a home's power wiring [59]. 
In the data collection procedure of this work, each electrical device of data set was actuated on and off five to six times individually in order to record a unique turn on transient signature of an electrical device. Moreover in order to record the simultaneous transient events random electrical devices are actuated such as turning on the DVD player, digital cable box, a gaming console and then a TV to simulate a TV watching experience through a universal remote. Events generated from these tasks were manually labelled. In the second step, feature extraction phase, Fourier features of the EMI signals in the $36-500 \mathrm{kHz}$ range are extracted. Finally, for classification approaches, a KNN-based classifier using a 10-fold cross validation is used. Results of this work showed $93.82 \%$ overall accuracy to identify the usage of individual electrical devices [44][59].

\subsubsection{Chang and Lee method}

Chang and Lee have developed a NILM approach [26][60][61][62], that can be applied for different electrical devices with the same real and reactive power. This method uses switching on transient energy along with steady state features in order to improve the recognition accuracy and computational speed of NILM results. The presented method is based on the fact that the switching on transient energy can be used as a unique feature to monitor commercial and industrial electrical devices [61]. 
The application of only steady-state parameters, i.e., real power and reactive power can limit the use of NILM systems for electrical device recognition. In this work, switching on transient signatures of electrical devices are incorporated into NILM to improve load identification accuracy within multiple operations. Combining steady-state and transient signatures can play an important role to improve recognition accuracy of NILM systems. In this study, artificial neural networks (ANN) and the computer supported cooperative work techniques (CSCW) are used to identify electrical devices [26][61]. The experimental results indicated that combining switching on transient features and steady-state power features is a useful tool for improving load recognition accuracy to $100 \%$ in a NILM system [60]. 


\section{Non-intrusive Load Monitoring System}

\section{Overview}

In this chapter, an overview of a NILM system is presented. According to the algorithm shown in Figure 3.1, a NILM system acquires the current and voltage signals, extracts features (signal characteristics), detects events, and classifies them [23][63][71].

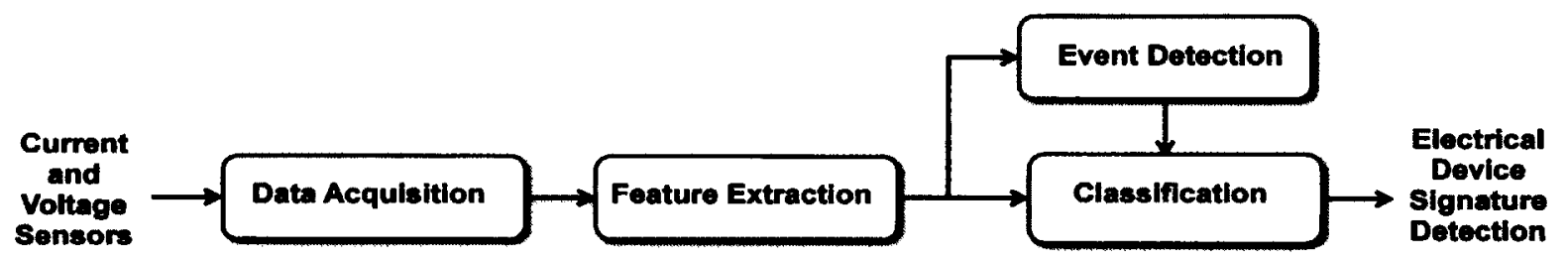

Figure 3.1: NILM algorithm [63]. 
A review of the general hardware setup and measuring equipment for data acquisition is provided in Section 3.1. The feature extraction step is described in Section 3.2. Event detection and classification methods used in this work are explained in sections 3.3 and 3.4 , respectively.

\subsection{Data Acquisition}

In this work, a NILM system was constructed to monitor a series of everyday use residential electrical devices connected to a common power bar (see Figure 3.2). As discussed in Chapter 2, the main objective of NILM systems is to monitor the usage of electrical devices from one single point at the main electric entrance without attaching separate sensors to each individual electrical device [43]. The experimental setup presented here represents a simplification of an actual household. Electrical monitoring is done at a single point coming into the power bar while multiple electrical devices can be operated from the power bar.

Electrical devices can be divided into three different classes according to their operation modes [43][45]: two-state devices, multi-state devices, and continuously varying loads. In order to simplify the experiments for developing our prototype NILM system, continuously varying loads that do not consume a constant power (e.g., washing machine and stove) are not monitored in this work. For the purpose of this project, we only consider 
two-state (ON/OFF) electrical devices and multi-state electrical devices in only one of their operation states (a single type of ON state) [64].

Data were obtained using a current transducer and a voltage transformer to measure instantaneous current and instantaneous voltage at the input of the power bar. A data acquisition device (DAQ) digitizes the analog voltage and current signals, which are then stored on a computer for offline analysis using Matlab. Additional information regarding the current and voltage sensors and analog-to-digital converter are provided in the following subsections.

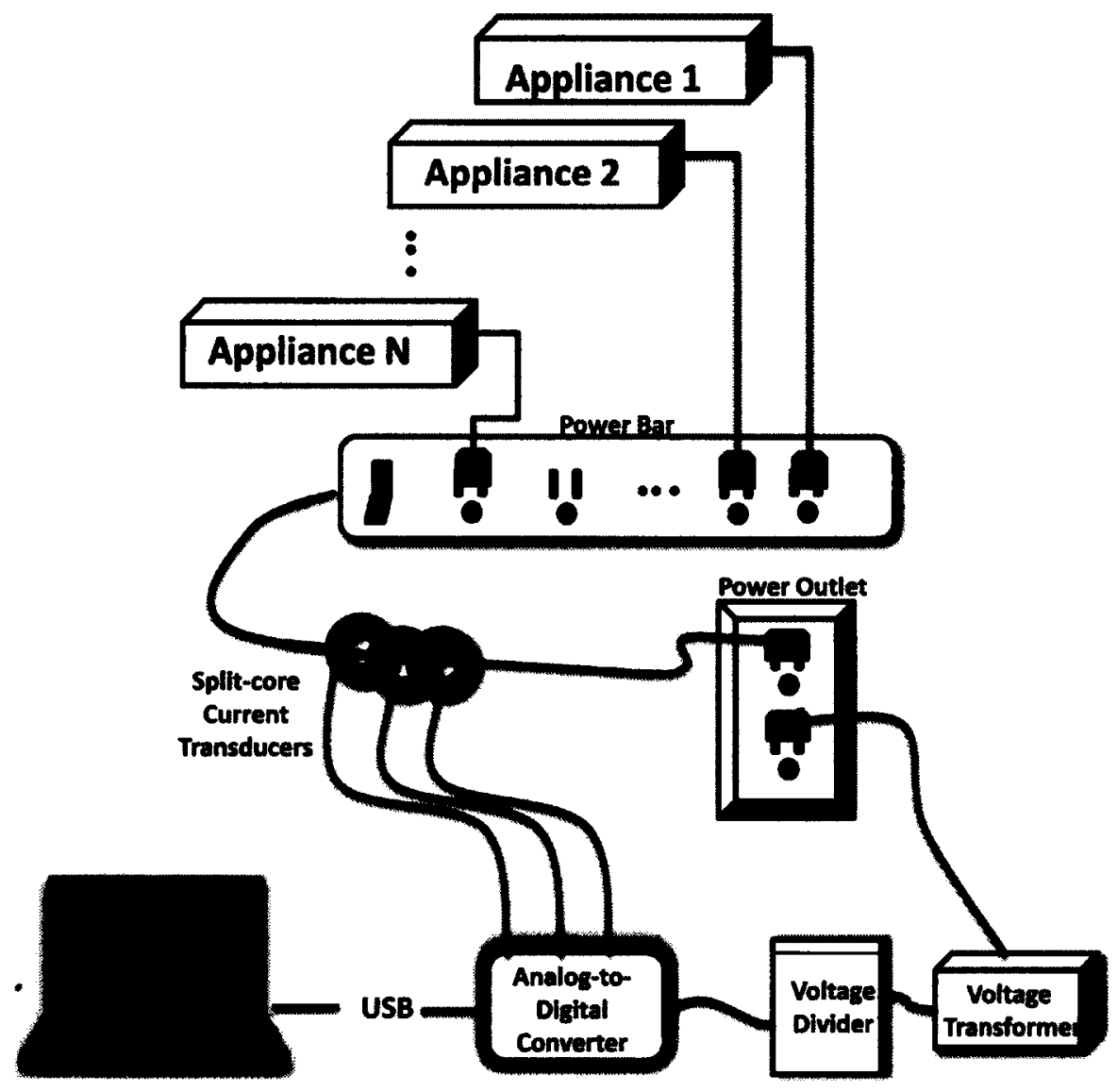

Figure 3.2: Experimental setup for our prototype NILM system. 
Figure 3.3 shows the experimental hardware used in this work for data acquisition.

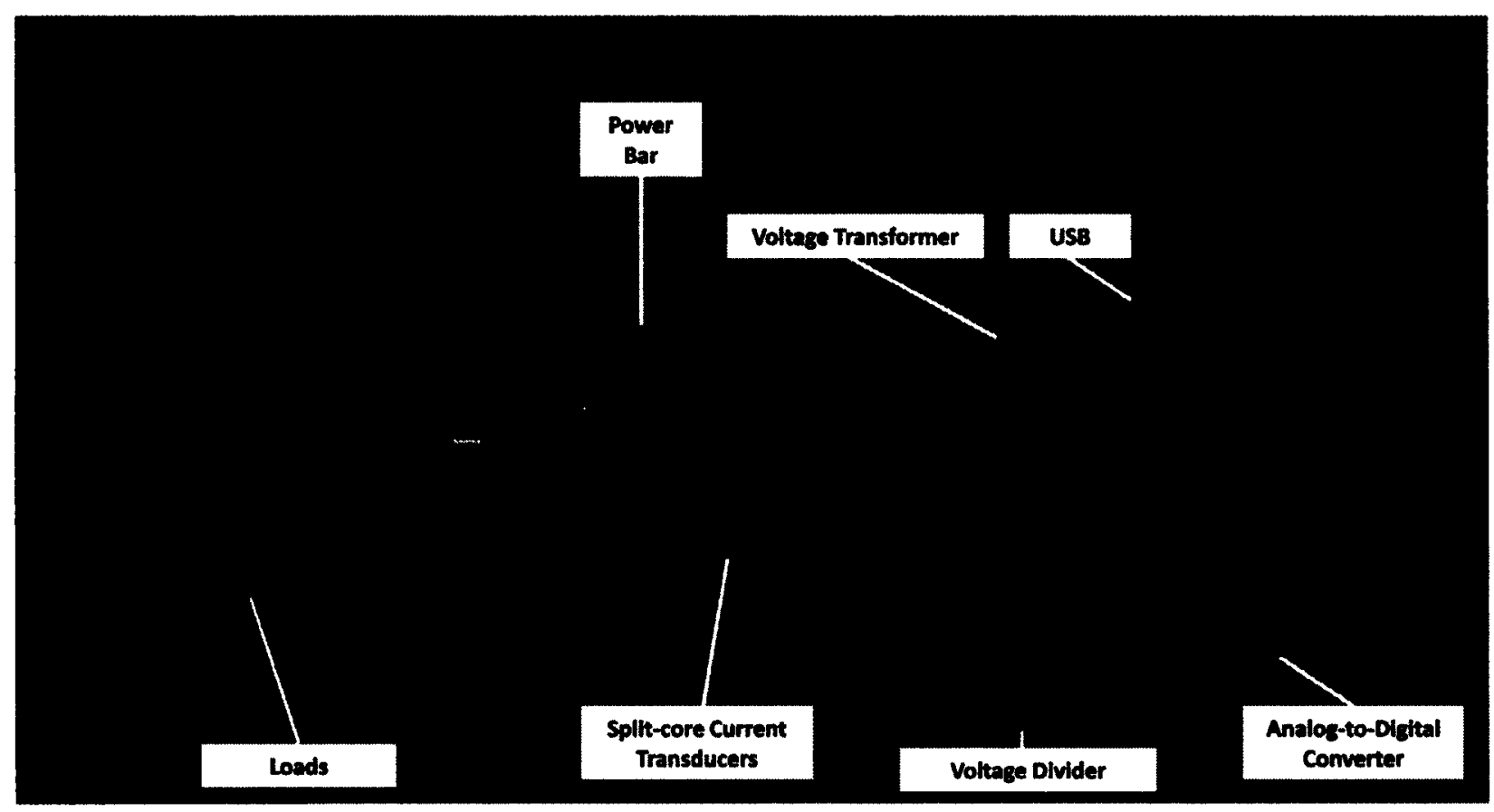

Figure 3.3: Experimental hardware used for data acquisition and data analysis.

\subsubsection{Current Sensor}

Split core current transducers offer a cost-effective and efficient method by which current can be measured without the need to severe the electrical conduction path, thereby reducing installation and commissioning time. They are ideally suited for use on installations where supply interruption is not desired [66]. Simultaneously, split-core current transducers do not disrupt the continuity of the electrical system maintaining the safety of the electrical system. Current measurements in this work were obtained using split-core AC current sensors applied to the live electrical wire feeding the power bar. 
The Magnelab split-core current transducer outputs a voltage (maximum $0.333 \mathrm{VAC}$ ) that is linearly proportional to the current [65]. Three split-core current transducers (Magnelab, Longmont CO, USA; models SCT-0400-005, SCT-0400-010, and SCT-0400-020), each with a different maximum current rating (5 A, $10 \mathrm{~A}$, and $20 \mathrm{~A}$ ), were used in the prototype NILM setup to enable accurate monitoring over the current range ( 0 to $20 \mathrm{~A}$ ). As SCT-0400 current transducers output 0.333 volt $A C$ at rated current, output voltage values were multiplied by (maximum current rating/0.333) and instantaneous current values were extracted. During a measurement the transducer with the highest sensitivity (i.e., lowest maximum current rating), which did not reach its maximum rating, was automatically selected for the current measurement. This ensures the highest resolution, while avoid sensor over ranging; an important feature given the wide range in current requirements in electrical devices. Our prototype NILM system is designed to monitor relatively low-powered electrical devices; therefore, it is not able to properly account for high-powered electrical devices due to the limitation in current sensor's measurement range.

\subsubsection{Voltage Sensor}

When a voltage sensor is added to the experimental setup, the instantaneous voltage measurements are used with the instantaneous current measurements to derive the real power and reactive power 
waveforms. In this work, a voltage transformer (Hammond Manufacturing, Center Tap power transformer, Model 166L10) was used to step down the 120 VAC voltage waveform to a 10 VAC output. A voltage divider circuit as shown in figure $3.4\left(R_{1}=100 \mathrm{~K}\right.$ and $\left.\mathrm{R} 2=4.7 \mathrm{~K}\right)$, was used to further step down the voltage waveform to a range comparable to the output of the current transducer $(0.45 \mathrm{VAC})$. Voltage values were recorded from $\mathrm{R}_{1}$. The recorded values (after analog-to-digital conversion - see section 3.1.3) were multiplied by (_ _ ) to obtain the instantaneous voltage values.

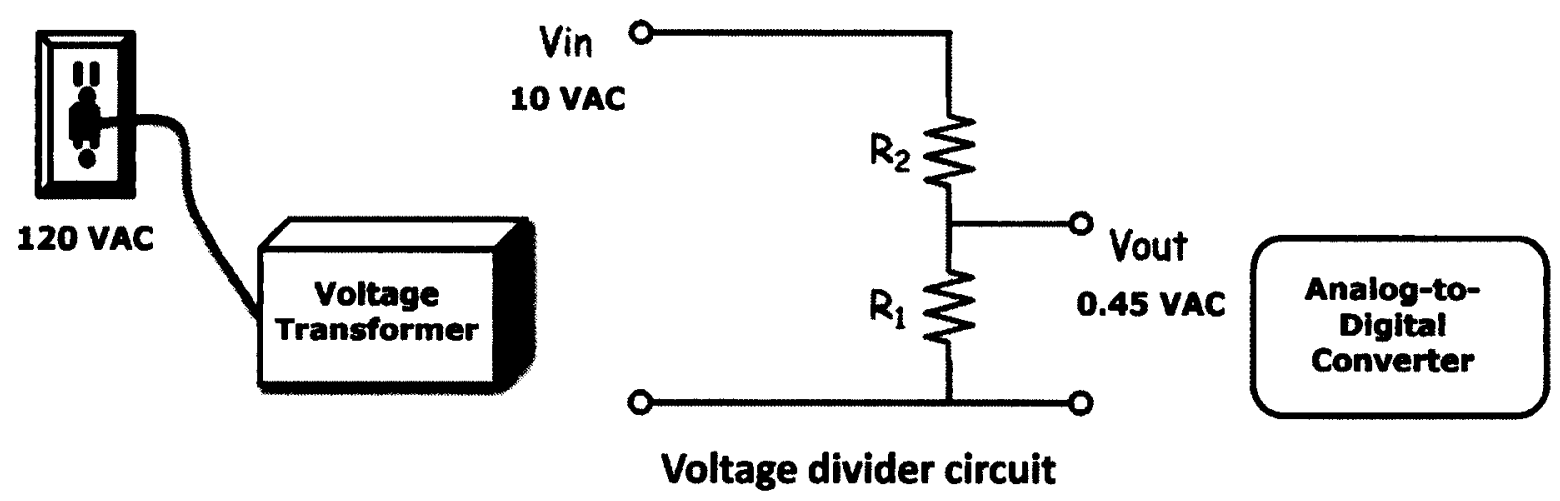

Figure 3.4: Voltage sensing in the NILM experimental setup.

\subsubsection{Analog-to-Digital Converter}

NI USB-6008 and NI USB-6216 data acquisition devices (National Instruments (NI), Austin TX, USA) were used for performing the data acquisition in this thesis. The instantaneous current and voltage (described in sections 3.1.1 and 3.1.2, respectively) were digitized using the analog-to- 
digital converter with software written in MATLAB using the Data Acquisition Toolbox.

The NI USB-6008 was used for steady-state analysis of our prototype NILM system (Chapter 4); current and voltage waveforms were digitized at the sampling rate of $1 \mathrm{kHz}$. The voltage waveform was acquired using one of the analog input channels (differential channel) and the remaining 3 differential channels of NI USB-6008 were used for the acquisition of current waveforms. As mentioned before, 3 current transducers were used (see Section 3.1.1), hence, 3 differential channels were needed for the current waveforms. Each differential channel of NI USB-6008 is equipped with a 12 bit analog to digital convertor with the maximum sampling rate of $2.5 \mathrm{kS} / \mathrm{s}$ (maximum $10 \mathrm{kS} / \mathrm{s}$ total across all channels) [67].

Since, the higher sampling frequencies $(>8 \mathrm{KHz}$ ) are required for transient signatures acquisition, the NI USB-6216 was used for transient signatures analysis in this work (Chapter 5). The NI USB-6216 offers 16 analog inputs, 8 differential channels, and a maximum total sampling rate of $400 \mathrm{kS} / \mathrm{s}$ across all channels [68]. Four differential channels of NI USB-6216 were used for data acquisition, one for voltage waveform recording and the remaining three for current waveform acquisition. In this work, for transient analysis, current and voltage waveforms were digitized using the sampling rate of $20 \mathrm{KHz}$. 


\subsection{Feature Extraction}

Representative features are computed from the raw sinusoidal current and voltage waveforms for further analysis. As discussed in Chapter 2, it has been demonstrated that using the real and reactive power features can track the usages of electrical devices [14], [43]. Higher harmonics of real power and reactive power can also be used for load identification [36][69]. A number of additional features have been proposed in the recent NILM works to improve the electrical device recognition accuracy [70]. In an investigation by Liang et al. a multi-feature approach was used to cover steady-state and transient behaviors of electrical devices. These features include: current waveform (CW), active/reactive power (PQ), harmonics (HAR), instantaneous admittance waveform (IAW), instantaneous power waveform (IPW), eigenvalues (EIG), and switching transient waveform (STW) [27]. In our work, we make use of the current and voltage signals to extract unique features such as root mean square (RMS) current (ARMS), RMS voltage (VRMS), real power (W), reactive power (VAR), apparent power (VA), power factor, admittance (Y), and Total Harmonics Distortion (THD). For the experiments described in this work, we focused only on the real power and reactive power. 


\subsubsection{Feature-Additive Criterion}

In order to decompose the total load into its components, we need features of individual electrical devices and their combination. Extracted features from current and voltage waveforms for a load signature analysis should preferably meet the feature-additive criterion [27]. Steady-state signatures are additive when two happen coincidentally (considering that electrical devices are wired in parallel) while in contrast transient properties are not additive [43]. The additive property of steady-state signatures makes it possible to analyze the aggregate signals (the signal for simultaneous operation of multiple devices) properly, when the sum of signatures is received (detected in the total load). For example, the turning on a $600 \mathrm{~W}$ computer LCD monitor while a $60 \mathrm{~W}$ incandescent lamp is in results in a 600 W step increase being detected in the total load (i.e., a total of $660 \mathrm{~W}$ ).

Feature additive criterion is stated in a work done by Liang et al. as follows: Suppose that $f_{i, j}$ is the feature $j$ of electrical device $i$ and $\Omega_{j}(t)$ is the aggregation of feature $j$ from a composite load (simultaneous operation of $K$ electrical devices) at time $t$. If electrical device $e$ is turned on at time $t+\Delta t$, and

$$
\left(\Omega_{j}(t+\Delta t)=\Omega_{j}(t)+f_{e, j}=\left(\sum_{i=1}^{K} f_{i, j}\right)+f_{e, j}\right.
$$

is satisfied, then feature $j$ meets the feature additive criteria [27]. 
If a feature can meet the feature-additive criterion, it can be used for load disaggregation purposes. The individual features of an aggregated signal can then be compared directly with the known features in the database. Loads may still be identified from aggregate signal if the feature does not meet the feature-additive criterion; however, the methods required would be more complex. In this work, the features chosen to be extracted from the current and voltage waveforms meet the feature additive criterion. Methods for load disaggregation presented in this work, assume that the feature-additive criterion is met.

\subsubsection{Steady State Load Signature}

Real power and reactive power are the most frequently used measurements for load identification analysis [27]. The key idea of using power features is that changes in the ON/OFF state of an electrical device can be detected by step changes in the total power signal [45].

Real power provides one of the most complete sets of information to explain load characteristics that can discriminate electrical devices of dissimilar consumption [43]. However, electrical devices may have similar real power consumption, making them difficult to discern using real power alone [26]. Device loads can be resistive, inductive, or capacitive according to its internal circuit [70]. For example, a kettle is almost purely resistive while a fan can be predominantly inductive. If the load is purely resistive, 
then the current and voltage signals are in phase. On the other hand, if the load consists of capacitive and/or inductive elements, it will affect the phase difference between current and voltage signals. In particular, for capacitive loads, the voltage is delayed with respect to the current while the contrary happens for inductive loads [23][70]. This effect is captured by the active and reactive power components, which, in mathematical terms, correspond to real and imaginary part of apparent power respectively, as shown in Figure $3.5[70]$.

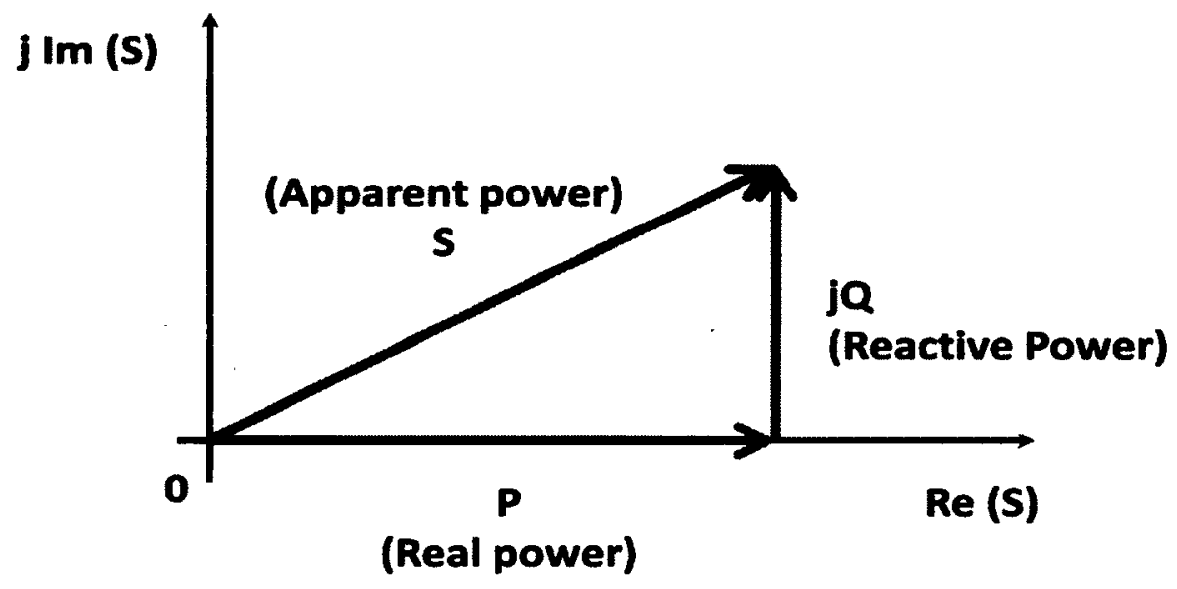

Figure 3.5: Relationship between reactive power $(Q)$ and active power $(P)[70]$.

In addition to the real power, the reactive power is also computed as a feature to establish the steady-state load signatures; the reactive power is the power associated with capacitive and inductive elements [23]. In general, electrical devices work through the real power (active), while the reactive power (passive) is due to the presence of storage elements in the electrical device circuit (inductors or capacitors) [70].The following equations 
report the relation between the instantaneous power (IPW $[n])$, active power $(P)$, apparent power (S), and reactive power $(Q)$ :

$$
\begin{gathered}
I P W[n]=i[n] \times v[n] \\
P[m]=\frac{1}{N} \sum_{(m-1) N}^{m N} I P W[n] \\
V_{R M S}[m]=\sqrt{\frac{1}{N} \sum_{(m-1) N}^{m N} v^{2}[n]} \\
I_{R M S}[m]=\sqrt{\frac{1}{N} \sum_{(m-1) N}^{m N} i^{2}[n]} \\
S[m]=I_{R M S}[m] \times V_{R M S}[m] \\
Q[m]=\sqrt{\left(S^{2}[m]-P^{2}[m]\right)}
\end{gathered}
$$

Where $i[n]=$ instantaneous current, $v[n]=$ instantaneous voltage, $I_{R M S}[m]=$ RMS current, and $V_{R M S}[m]=$ RMS voltage. In the above equations $N$ represents the length of an individual analysis window in number of samples. In this work, real power and reactive power features are computed from the current and voltage signals using a sliding analysis window with a time length $T(T=N /$ sampling frequency) [72]. An example of the non-overlapping analysis window is depicted in Figure 3.6, shown with window size of $T=$ $100 \mathrm{~ms}$ from instantaneous voltage waveform. 


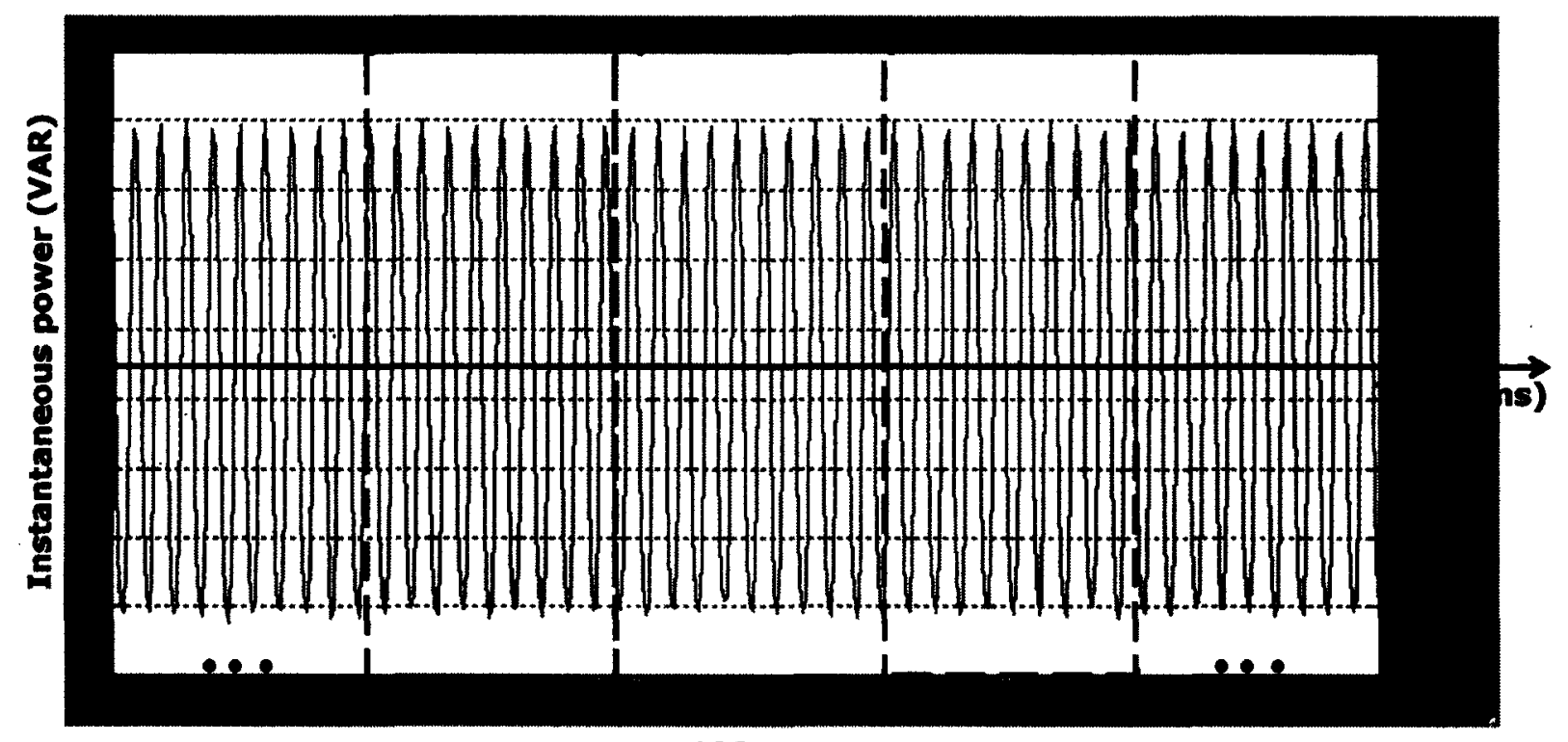

$100 \mathrm{~ms}$

Figure 3.6: An example of sliding analysis window for feature extraction. Real power values are extracted from each $100 \mathrm{~ms}$ analysis windows of instantaneous power.

Steady-State signatures used in these work, including real power and reactive power signatures meet feature additive criteria [27]. Figure 3.7 shows an example from our work using real power waveforms (a) and reactive power waveforms (b) when multiple electrical devices are operated simultaneously (a description of the experimental procedures used to obtain these data is in section 4.3). Real power and reactive power waveforms extracted in the feature extraction level are the inputs for the next step of NILM algorithm which is event detection. 


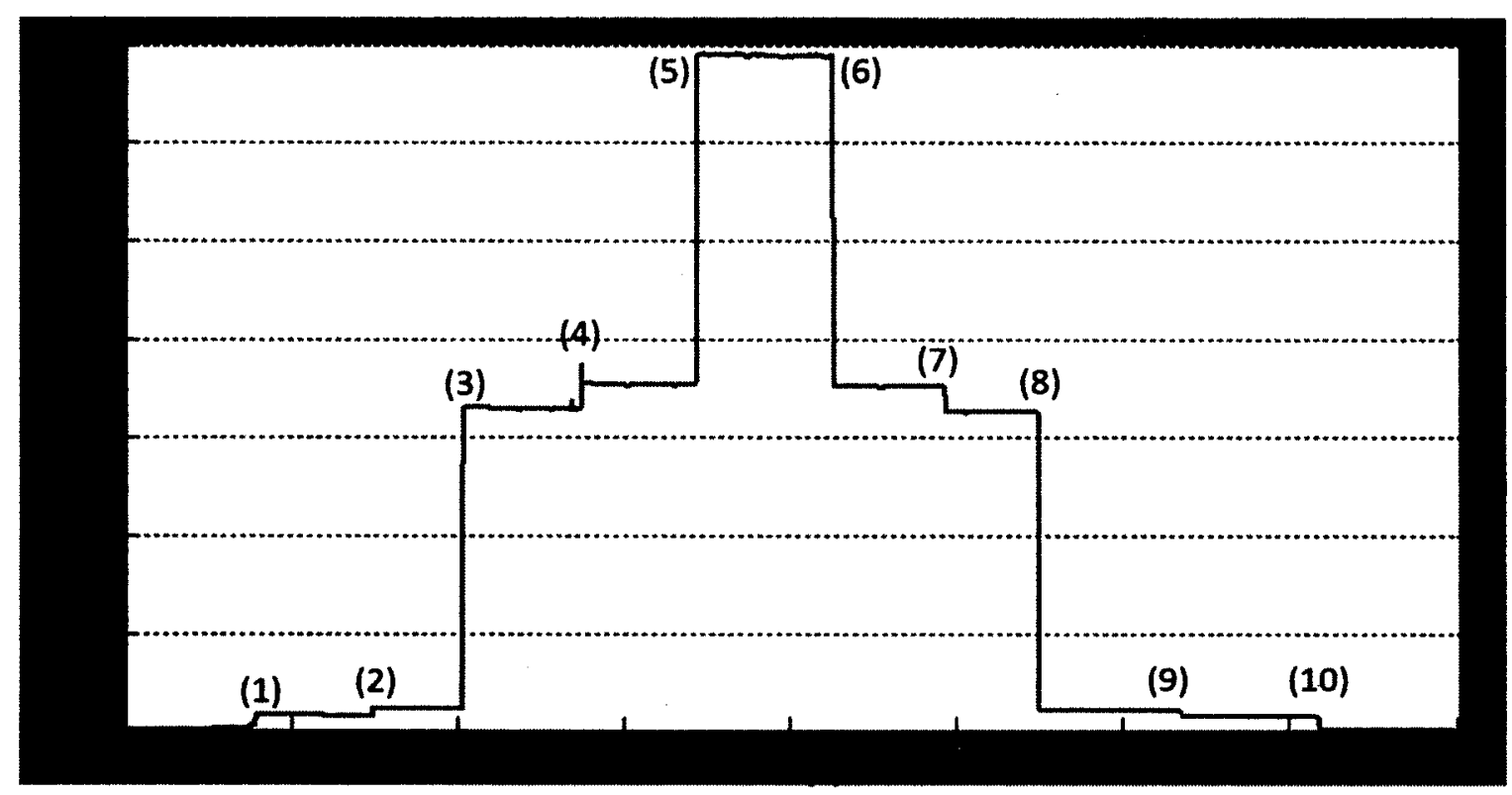

(a)

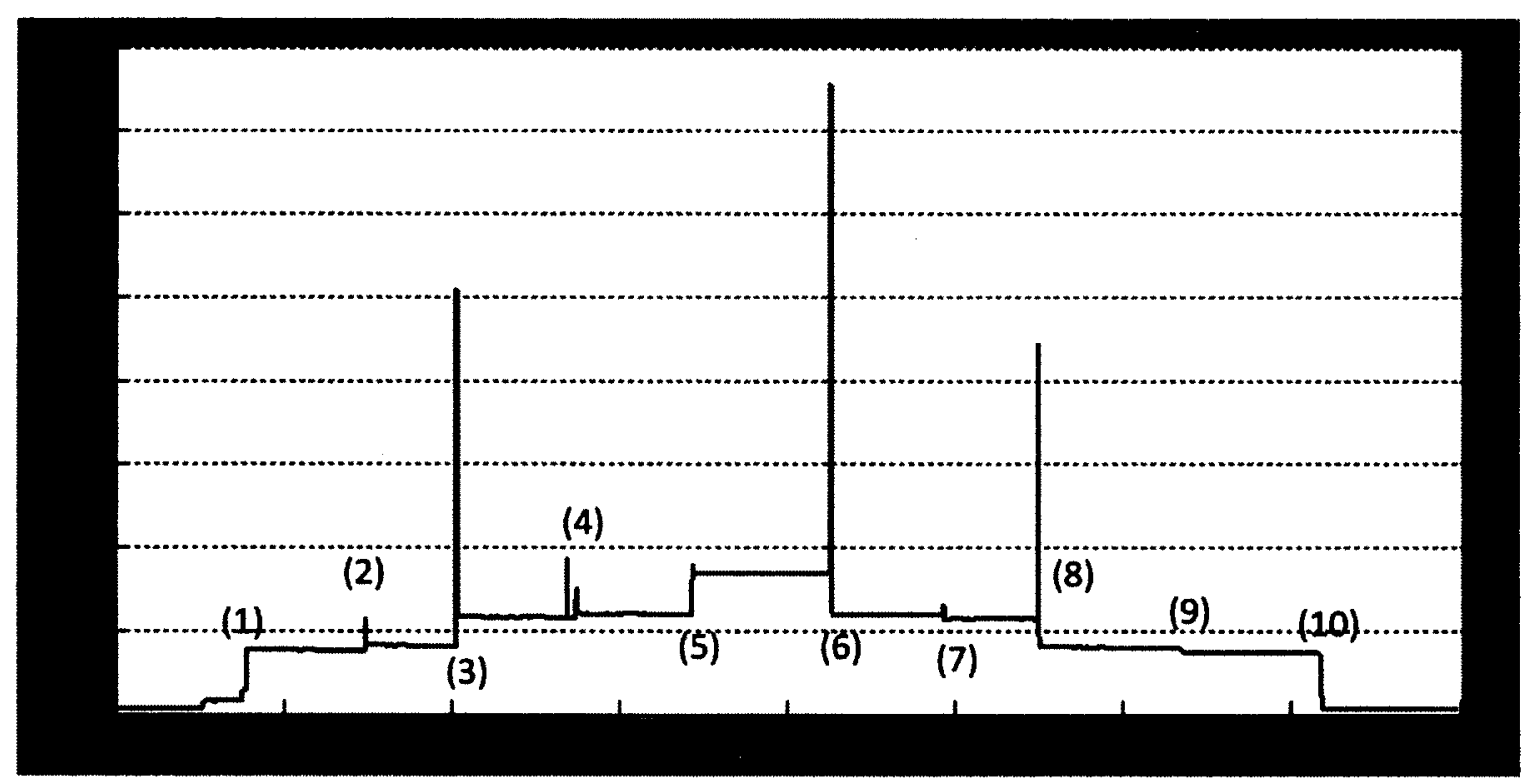

(b)

Figure 3.7: Examples of a composite real power waveform (a) and reactive power wave form (b) for multiple electrical devices operation. Numbers indicate events (i.e., electrical devices turning on or off): (1) Computer LCD monitor: ON, (2) Fluorescent lamp: ON, (3) Coffee maker: ON, (4) Incandescent lamp: ON, (5) Electric kettle: ON, (6) Electric kettle: OFF, (7) Incandescent lamp: OFF, (8) Coffee maker: OFF, (9) Fluorescent lamp: OFF, and (10) Computer LCD monitor: OFF. 


\subsubsection{Transient Load Signature}

It may not be possible to distinguish between two different electrical devices with the same real power and reactive power [26]. Switching transient waveforms (STW) are presented in this work to overcome this limitation. Transient signatures are captured using higher sampling frequencies than steady-state signatures. Therefore, they convey more information about the characteristics of an individual electrical device than steady-state signatures $[26][27]$.

Using STW for load identification is more difficult since a high sampling rate of the current and voltage signals is needed [44]. For this purpose, a sampling frequency of $20 \mathrm{kHz}$ is used in this work to capture transient signatures of electrical devices when they are switching on. In order to extract switching on waveforms, features are computed from overlapping sliding analysis window. An example of the sliding window is depicted in Figure 3.8, shown with analysis windows of $50 \mathrm{~ms}$ in length, spaced $1 \mathrm{~ms}$ apart. A single feature vector (i.e. real power) is produced from each analysis window. The real power value was computed from the current and voltage measurements in each analysis window. 


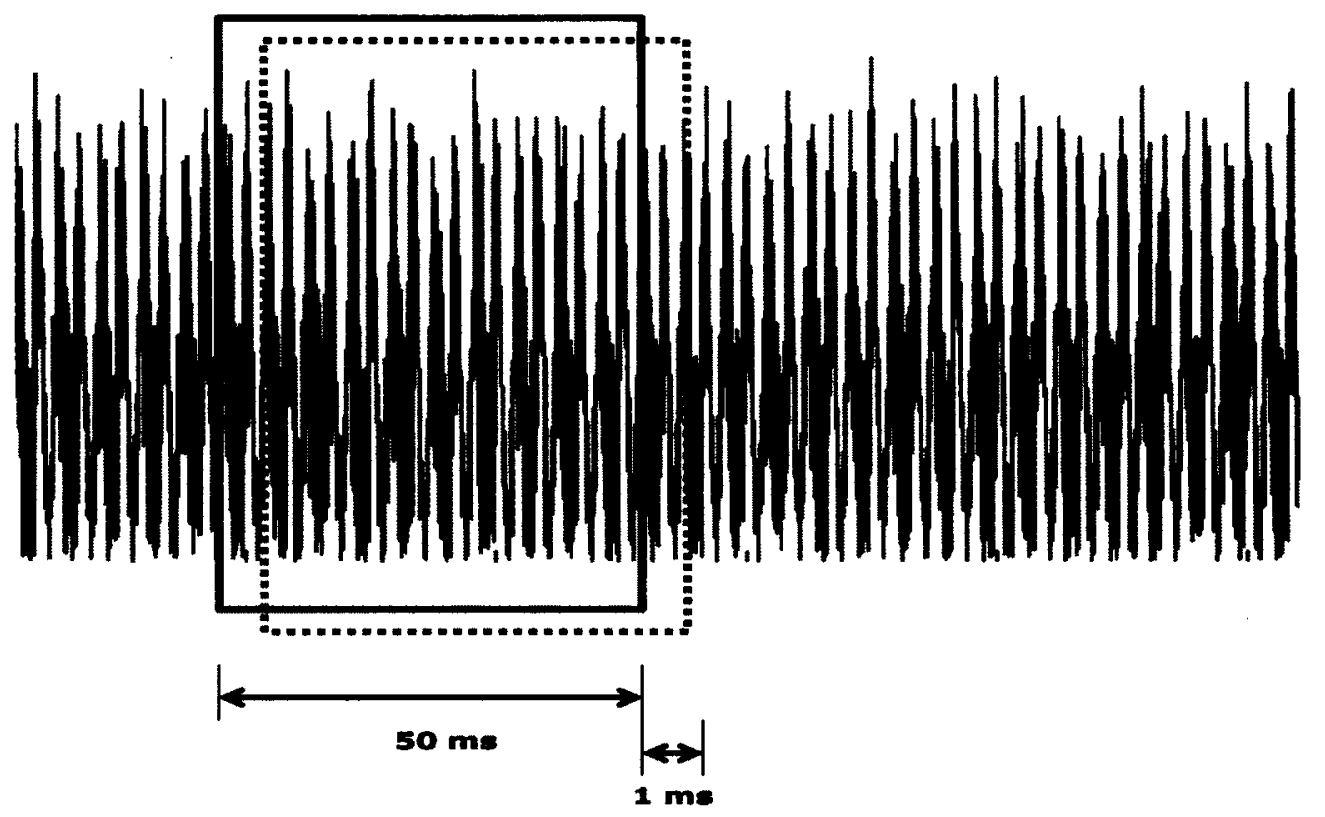

Figure 3.8: An example of sliding analysis window for STW extraction (based on [72]).

Figure 3.9 shows examples of the STW from three different electrical devices (a description of the experimental procedures used to obtain these data is in chapter 5). It is easy to observe that the hair dryer will increase from a zero to its steady-state value gradually without any overshoot. However, the kitchen blender and computer LCD monitor will have a sharp rise to a peak value and then drop to their steady-state values. 


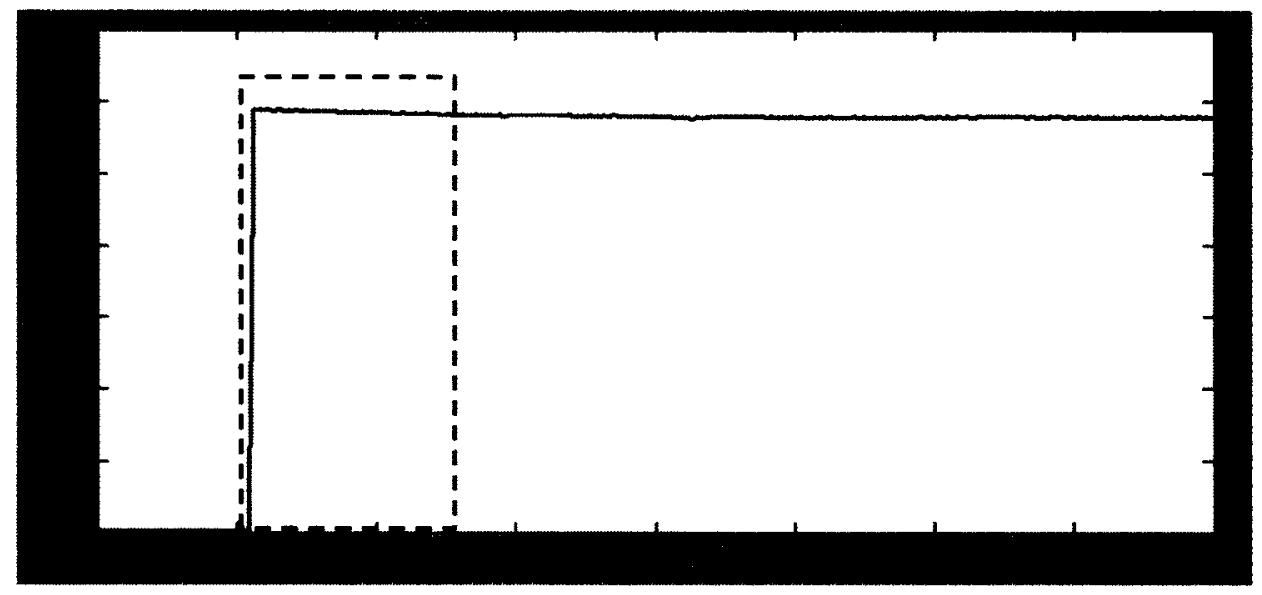

(a)

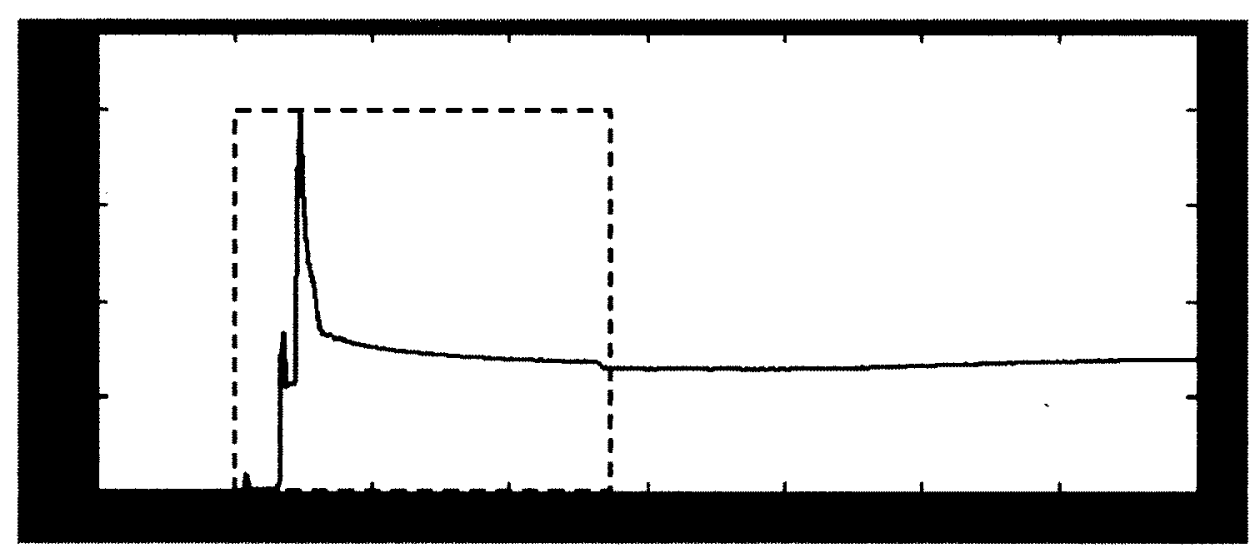

(b)

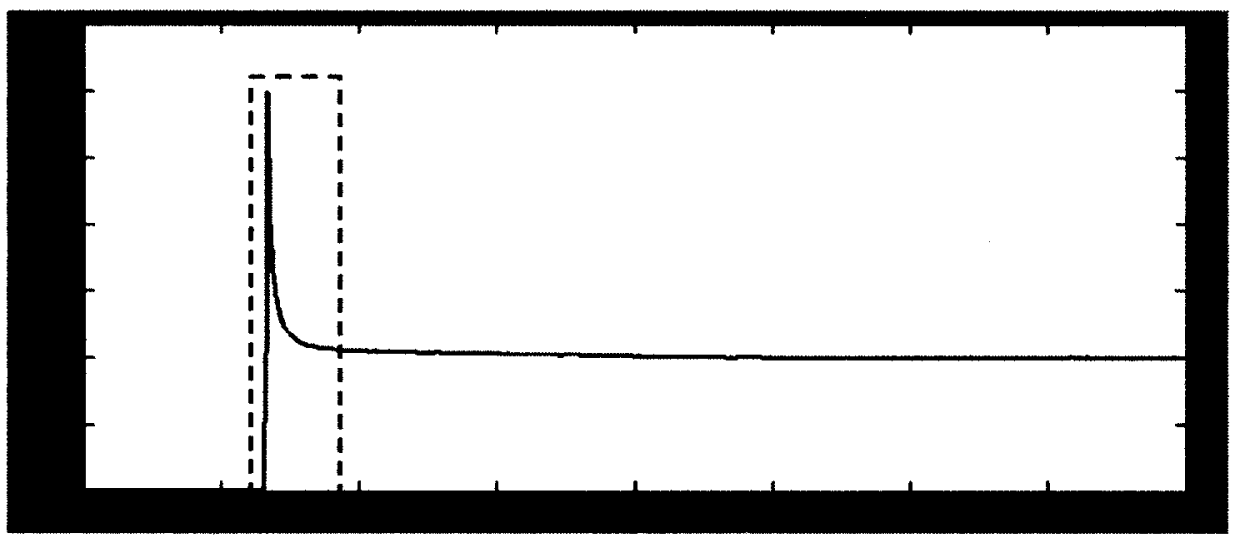

(c)

Figure 3.9: Transient data for electrical devices switching from off to on: (a) hair dryer, (b) computer CRT monitor, and (c) kitchen blender. 


\subsection{Event Detection}

Detecting a change in the extracted signals is important as a change could be related to a change in the consumption of a single electrical device or group of electrical devices (e.g., devices turning on or off); this is called event detection [23]. It is not a difficult task for a trained human to detect changes via visual inspection of values of features related to the operation of multiple electrical devices [23]; however, we need to develop algorithms to identify the times at which the values of extracted features change from one nearly constant value to another in an automated manner [28][36]. Many signal processing techniques such as filtering, differentiating, and peak detection, have been used for step-change detection [69][75][76].

The event detection method we have presented in this work used changes in real power as indicators of the state transitions. The real power waveform is the input to an event detection algorithm of our system which finds the times of all step changes. Our method is based on the assumption that when a step change occurs in a real power signature, one electrical device is being turned on or/and turned off [45].

We use a simple differentiating method for event detection step of our work. The algorithm is as follows: Firstly, a difference signal $d[n]$ is computed from the real power waveform $p[n]$. A window of consecutive samples of real power signal is selected; the mean value of each window is 
subtracted by the mean value of the next sliding window which has the same size. Mathematically, this difference signal can be described as:

$$
d[n]=\frac{1}{N} \sum_{i=n-N}^{n-1} p[i]-\frac{1}{N} \sum_{j=n}^{n+N-1} p[j]
$$

In this work $N=50$, which corresponds to $5 \mathrm{~s}$ as analysis windows are $100 \mathrm{~ms}$ in length. Secondly, events are located at local peaks of the absolute value of $d[n]$, which exceed a given threshold; in this work, the threshold was set as $5 \mathrm{~W}$. Finally, maximum values of the local areas (bigger than threshold) are found. These values represent the location of the step-change in the original aggregate real power waveform. It is noted that this event detection algorithm assumes that two electrical devices do not change state within $5 \mathrm{~s}$ of each other. Figure 3.10 shows the application of this algorithm.

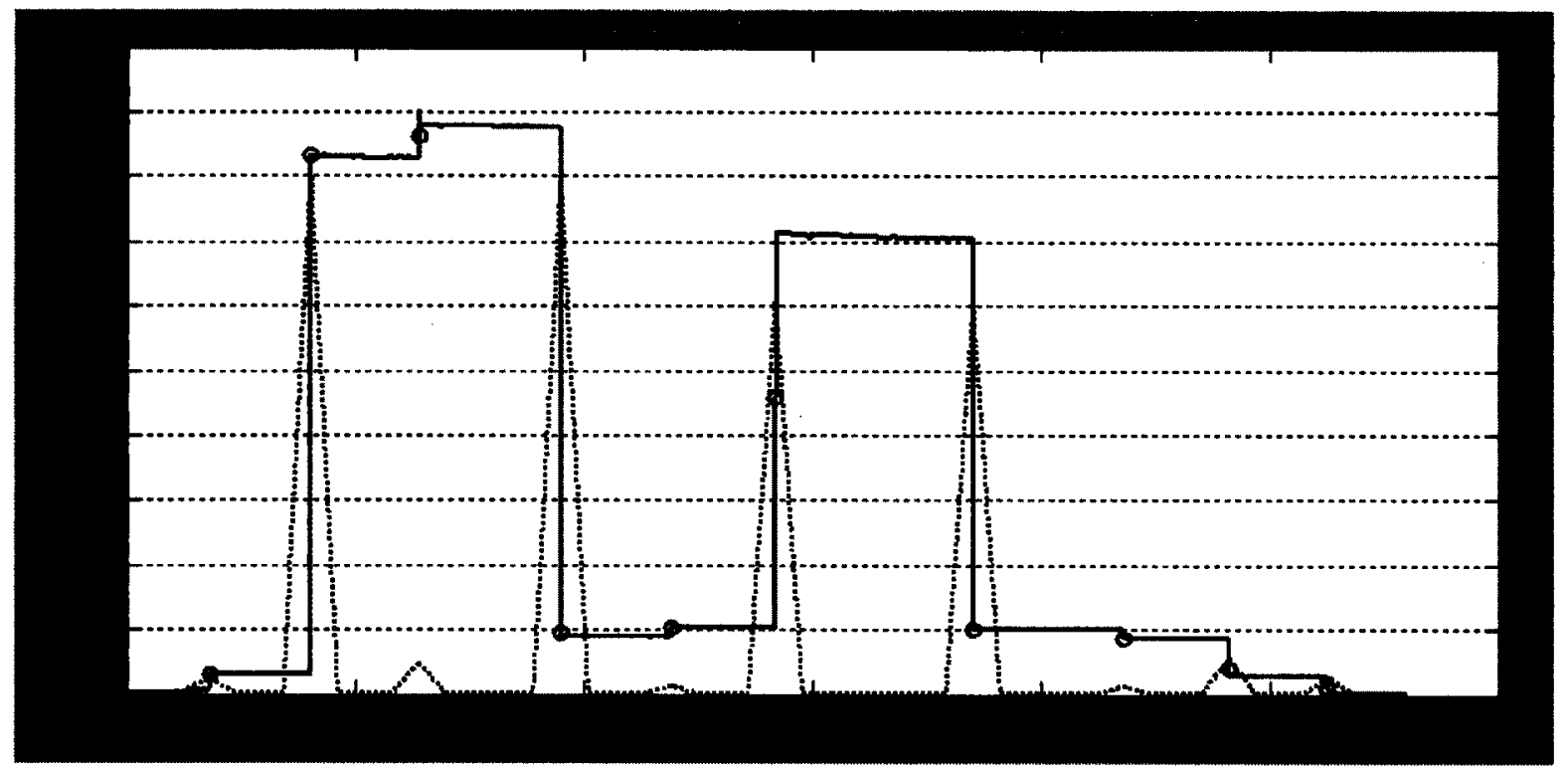

Figure 3.10: An example of event detection algorithm. (a) Heavy black line - original real power waveform of a composite load signature related to the multiple operations of 5 electrical devices is given as an input to the event detection algorithm; (b) dashed black line - difference waveform is derived by using an averaging window to determine the peak values; (c) red circles - These values represent the location of the step-change in the original aggregate real power waveform. 
The main obvious challenge arises for the attempted event detection method is setting the appropriate parameters for the size of sliding window and threshold. The first limitation of our event detection method is that, the electrical devices that switch on and off very quickly will be masked by the averaging window, and will not be identified. The second challenge is that, events that are captured in the same window are most likely going to be combined. Selecting a smaller window helps differentiate events from one another but results in lower noise rejection. Defining the size of this window and the threshold is critical and each combination of these leads will affect the accuracy of detected events [73].

\subsection{Pattern Recognition}

The objective of the pattern recognition algorithm is to assign a label to the collection of features previously extracted from the current and voltage waveforms, associated with each event that is detected [23]. As we discussed in chapter 2, many researchers have tried different pattern classification methods (e.g., neural networks, cluster matching) in NILM systems [17][30]. Almost all of the examined solutions were based on offline training of the algorithm [23]. Since continuous monitoring implies that more than one electrical device can be present at a time, either signal disaggregation needs to be used, or the detection system must be trained for all possible combinations of the electrical devices being switched on and 
off [44]. Srinivasan et al. [38] selected the latter, so that they needed to train their classifier using all $2^{8}=256$ possible ON/OFF combinations among the eight electrical devices used. The reported detection accuracy was about 80 to $90 \%$. Even though the implementation of such a system would appear to be straightforward, the system is not yet practical. A typical household includes 30 to 50 electrical devices which suggest a number of $2^{30}$ to $2^{50}$ of various ON/OFF combinations; this number is prohibitively large for any practical application [38][44].

In this work, pattern classification was performed using discriminant analysis using Mahalanobis distances [74]. Instead of discriminant analysis, in section 4.3, a $k$-nearest neighbour $(\mathrm{KNN})$ classifier $(k=1)$ was used for classification in order to determine which device turned on or off (higher electrical device recognition accuracies were achieved using the KNN classifier). A NETLAB toolbox (version 3.2) acquired from (http://www.ncrg.aston.ac.uk/netlab/down.php) that runs in MATLAB was used to implement KNN classification [81]. With a known electrical device database and the extracted features, this method will identify from the detected event list which electrical device has been switched on/off and when (at which time).

The output of the event detection step is the collection of indices representing the detected step-changes of the real power waveform. In order to perform the pattern classification step, the difference between the 
average value of the previous and subsequent constant values of each detected index are used to determine the magnitude of edges. The magnitude of each edge represents the change in the real power consumption of a single electrical device or group of electrical devices. The pattern classification assumes that for each detected event (small time interval), only one electrical device is changing states (either turning on or off) [43]; Therefore, there is no need to train the system for all possible combination of the electrical devices in this system. 


\section{Steady-State Analysis}

This chapter describes our steady-state based methodology for the NILM systems including data acquisition, feature extraction, pattern recognition, results, and discussion. Our steady-state analysis is broken down into three phases. In the first phase described in Section 4.1, our method for classification of single electrical devices is presented. Section 4.2 reviews our work's second phase for identification of two electrical devices when operating simultaneously. We have extended the load identification method used in Section 4.2 for determining the operating schedule of five electrical devices operating simultaneously; the third phase is described in Section 4.3. Finally, the conclusion for steady-state experiments of our work is presented in Section 4.4. 


\subsection{Single Electrical Device Classification}

A NILM system (Figure 4.1) was constructed to monitor the usage of seven electrical devices connected to a common power bar. Our prototype NILM system monitors current and voltage signals at the input of the power bar to simulate the NILM systems capability to monitor at a single electrical source. Experimental testing of our NILM prototype in this section is performed using seven common household electrical devices, where only one device was one at a time. Our experimental results for single electrical device recognition of seven devices showed that our proposed approach can achieve $100 \%$ classification accuracy.

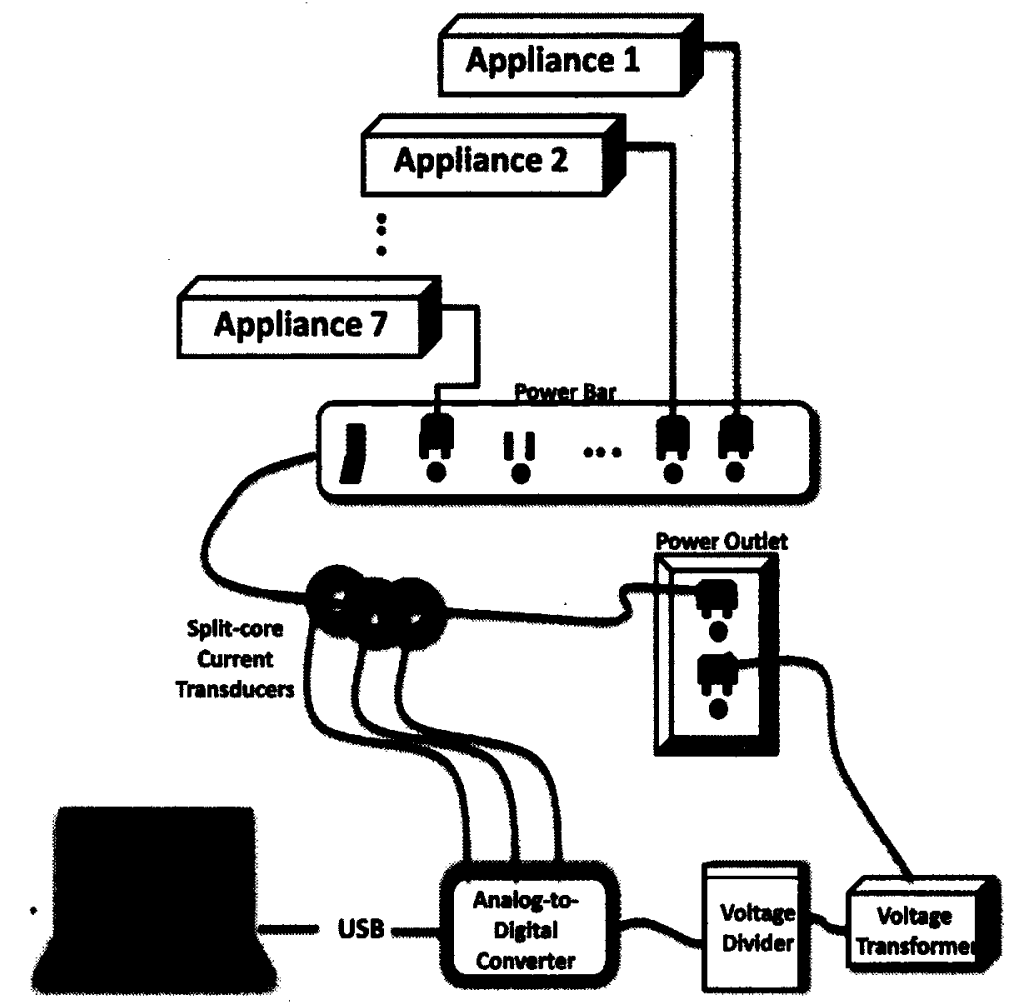

Figure 4.1: Experimental setup for our NILM prototype (Load identification during the individual operation of seven electrical devices). 


\section{Data Acquisition}

Seven household electrical devices (Table 4.1) were used as loads in this section (see Appendix B for the specifications of the electrical devices used in this work). To establish load signatures, each device was operated mutually exclusive of one another (i.e., electrical devices were not operated simultaneously). In this work, we consider electrical devices in one of two states (i.e., ON and OFF). Multi-state electrical devices (e.g., microwave had different power levels) were used in only one of their operational states. Data were collected for each device from its ON state for 10 trials, with each measurement lasting 5 seconds. Data were collected during its steady state operation; that is, data collection commenced after the device under test was turned on. A total of 70 measurements were completed $(7$ devices $\times 10$ trials).

\subsubsection{Feature Extraction}

Each 5-second measurement was broken down into non-overlapping $100 \mathrm{~ms}$ analysis windows (50 analysis windows per measurement). The real power value was computed from the current and voltage measurements in each analysis window. As mentioned in chapter 3, real power provides one of the most complete sets of information to explain load characteristics [45]. However, electrical devices may have similar real power levels, making them difficult to discern using real power alone [24]. In addition to the real power, 
the reactive power is also computed as a feature from each analysis window to establish the steady-state load signatures; the reactive power is the power associated with capacitive and inductive elements (see section 3.2.2).

\subsubsection{Pattern Recognition}

Pattern classification was performed using discriminant analysis using Mahalanobis distances [74]. Classifier training was performed using 1 trial and classifier testing was performed using the remaining 9 trials. Thus, the training data and testing data are mutually exclusive of one another. Data used for confusion matrices in results sections are from testing sets. Crossvalidation was performed by repeating the training and testing 10 times, such that each trial was used for training. There were a total of 31,500 test cases (10 repetitions $\times 9$ test trials $\times 50$ analysis windows $\times 7$ devices).

\subsubsection{Results}

Table 4.1 lists the mean real power and reactive power for all the electrical devices ( \pm one standard deviation). Table 4.2 and Table 4.3 show the classification confusion matrices for the real power and reactive power, respectively (results are for the testing data only; i.e., classification results for the training data are not included in the confusion matrices). The mean classification accuracies were $\mathbf{8 8 . 2 2 \%}$ and $\mathbf{8 5 . 7 9 \%}$ for real power and reactive power, respectively. When using the real power and reactive power, 
the classification accuracy increases to $100 \%$; the associated confusion matrix is shown in Table 4.4.

Table 4.1: Electrical devices used in experimental setup, along with their mean real power and reactive power ( \pm standard deviation), computed across all analysis windows.

\begin{tabular}{c|c|c|c}
\hline Load & Devices & $\begin{array}{c}\text { Real Power } \\
\text { (Watt) }\end{array}$ & $\begin{array}{c}\text { Reactive Power } \\
\text { (VAR) }\end{array}$ \\
\hline 1 & Microwave & $802.1 \pm 21.81$ & $214.14 \pm 8.08$ \\
\hline 2 & Electric kettle & $785.66 \pm 5.82$ & $84.59 \pm 1.19$ \\
\hline 3 & Coffee maker & $602.02 \pm 4.57$ & $65.56 \pm 0.92$ \\
\hline 4 & Laptop charger & $65.95 \pm 0.64$ & $84.69 \pm 2.01$ \\
\hline 5 & Incandescent lamp & $58.44 \pm 0.39$ & $9.09 \pm 0.12$ \\
\hline 6 & Computer LCD monitor & $28.71 \pm 0.38$ & $36.04 \pm 0.51$ \\
\hline 7 & Fluorescent lamp & $18.47 \pm 1.44$ & $26 \pm 2.81$ \\
\hline
\end{tabular}

Table 4.2: Classification confusion matrix for real power (Mean classification accuracy: $88.22 \%)$.

\begin{tabular}{|c|c|c|c|c|c|c|c|c|}
\hline & \multicolumn{7}{|c|}{ Predicted load } \\
\hline & & Load 1 & Load 2 & Load 3 & Load 4 & Load 5 & Load 6 & Load 7 \\
\hline \multirow{7}{*}{ 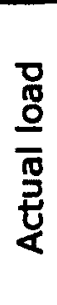 } & Load 1 & 3604 & 896 & 0 & 0 & 0 & 0 & 0 \\
\hline & Load 2 & 2704 & 1796 & 0 & 0 & 0 & 0 & 0 \\
\hline & Load 3 & 0 & 0 & 4500 & 0 & 0 & 0 & 0 \\
\hline & Load 4 & 0 & 0 & 0 & 4500 & 0 & 0 & 0 \\
\hline & Load 5 & 0 & 0 & 0 & 111 & 4389 & 0 & 0 \\
\hline & Load 6 & 0 & 0 & 0 & 0 & 0 & 4500 & 0 \\
\hline & Load 7 & 0 & 0 & 0 & 0 & 0 & 0 & 4500 \\
\hline
\end{tabular}

Table 4.3: Classification confusion matrix for reactive power (Mean classification accuracy: $85.79 \%)$.

\begin{tabular}{|c|c|c|c|c|c|c|c|c|}
\hline & \multicolumn{7}{|c|}{ Predicted load } \\
\hline & & Load 1 & Load 2 & Load 3 & Load 4 & Load 5 & Load 6 & Load 7 \\
\hline \multirow{7}{*}{$\frac{\text { 몽 }}{\frac{10}{8}}$} & Load 1 & 4500 & 0 & 0 & 0 & 0 & 0 & 0 \\
\hline & Load 2 & 0 & 2269 & 0 & 2231 & 0 & 0 & 0 \\
\hline & Load 3 & 0 & 0 & 4500 & 0 & 0 & 0 & 0 \\
\hline & Load 4 & 0 & 1786 & 0 & 2714 & 0 & 0 & 0 \\
\hline & Load 5 & 0 & 0 & 0 & 0 & 4500 & 0 & 0 \\
\hline & Load 6 & 0 & 0 & 0 & 0 & 0 & 4041 & 459 \\
\hline & Load 7 & 0 & 0 & 0 & 0 & 0 & 0 & 4500 \\
\hline
\end{tabular}


Table 4.4: Classification confusion matrix for real power and reactive power (Mean classification accuracy: $100 \%$ ).

\begin{tabular}{|c|c|c|c|c|c|c|c|c|}
\hline & \multicolumn{7}{|c|}{ Predicted load } \\
\hline & & Load 1 & Load 2 & Load 3 & Load 4 & Load 5 & Load 6 & Load 7 \\
\hline \multirow{7}{*}{ 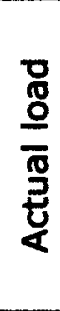 } & Load 1 & 4500 & 0 & 0 & 0 & 0 & 0 & 0 \\
\hline & Load 2 & 0 & 4500 & 0 & 0 & 0 & 0 & 0 \\
\hline & Load 3 & 0 & 0 & 4500 & 0 & 0 & 0 & 0 \\
\hline & Load 4 & 0 & 0 & 0 & 4500 & 0 & 0 & 0 \\
\hline & Load 5 & 0 & 0 & 0 & 0 & 4500 & 0 & 0 \\
\hline & Load 6 & 0 & 0 & 0 & 0 & 0 & 4500 & 0 \\
\hline & Load 7 & 0 & 0 & 0 & 0 & 0 & 0 & 4500 \\
\hline
\end{tabular}

\subsubsection{Discussion}

High classification accuracy $(88.22 \%)$ is achieved using the real power. This is not unexpected given the high repeatability of the real power measurements, indicated by the low standard deviation values in Table 4.1. Table 4.2 indicates that load 1 (microwave) and load 2 (electric kettle) are the electrical devices that are misclassified most frequently; these devices were misclassified as each other and have comparable real power values, as well as the highest standard deviation values (Table 4.1).

Electrical devices that have similar real power values may still be discernible through the reactive power. Using just the reactive power, a classification accuracy of $\mathbf{8 5 . 7 9 \%}$ was achieved. Classification accuracy is lower than real power. Table 4.3 indicates that the majority of the misclassifications are load 2 (electric kettle) and load 4 (laptop charger) being misclassified as each other. Table 4.1 shows that these devices have similar reactive powers, which explains the misclassification. 
From Table 4.4, it can be seen that using both real and reactive power, the classification accuracy increases to $100 \%$ for the dataset used in this work. Although some of the electrical devices had similar real powers or similar reactive powers, devices were dissimilar when real and reactive powers were considered simultaneously, enabling the increased classification accuracy.

\subsection{Classification of two electrical devices operating simultaneously}

This section reviews our NILM prototype system for identification of five common household electrical devices when two are operating simultaneously. This work proposes a feature based model using only real power to recognize the usage of electrical devices; the method is generalizable to include other features that mean the feature additive criterion (e.g., reactive power). A NILM system (Figure 4.2) was constructed to monitor the usage of two different electrical devices ( $A$ and $B$ ) connected to a common power bar. Experimental results of this work showed that the proposed approach can achieve $95 \%$ device recognition accuracy for five electrical devices operating simultaneously in pairs. 


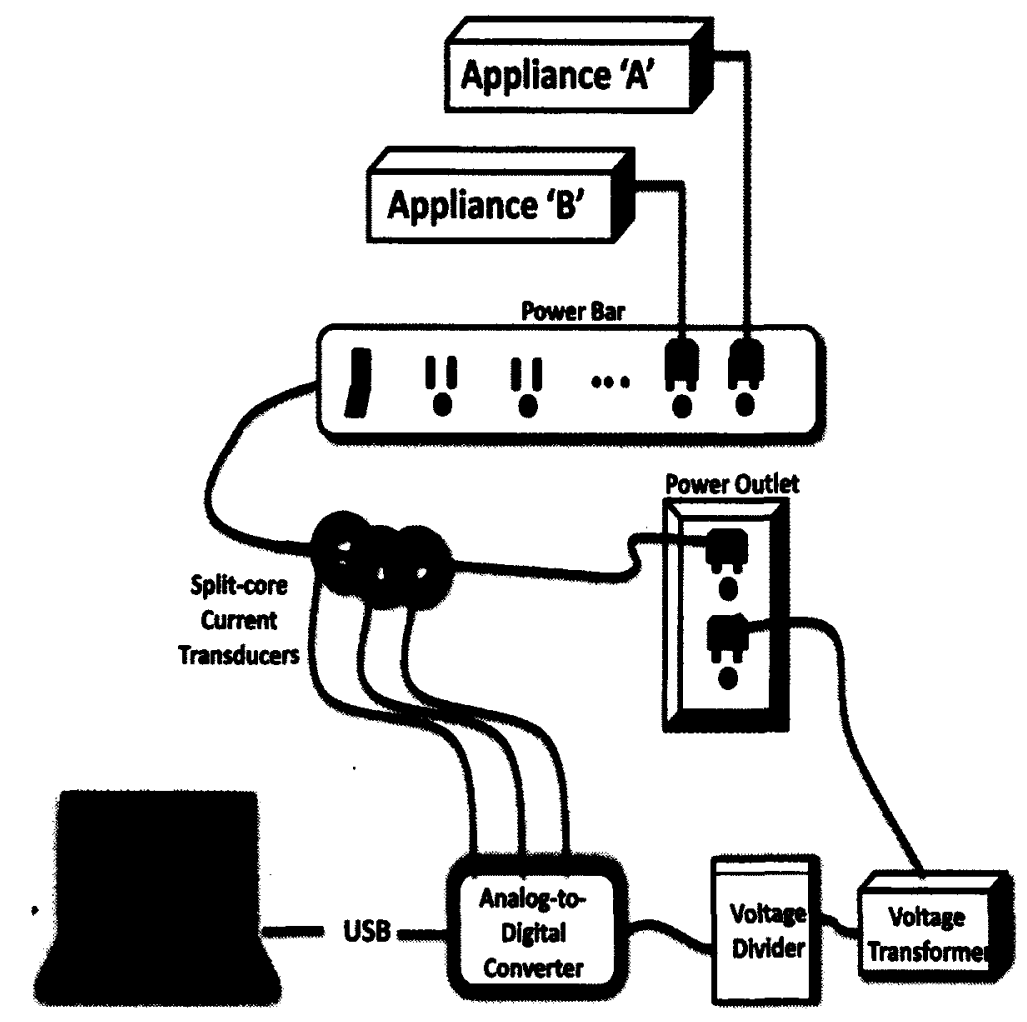

Figure 4.2: Experimental setup for our NILM prototype (Load identification during the simultaneous operation of two electrical devices).

\subsubsection{Data Acquisition}

Five household electrical devices were used as loads in this section: electric kettle (Load 1), coffee maker (Load 2), computer LCD monitor (Load 3), and incandescent lamp (Load 4), and fluorescent lamp (Load 5). The new set of electrical devices used in this section is a subset of the seven common household electrical devices used in section 4.1. In this work, we consider electrical devices in one of two states (i.e., ON and OFF). In this section, we are interested in the recognition of individual electrical devices when they are operating simultaneously. In other words, disaggregating the composite load signal into its components, which are the individual electrical devices. 
We focus on disaggregation of two devices operating simultaneously (disaggregation method for classification of the all five electrical devices are discussed in Section 4.3).

We have ten different combinations of two devices ( 5 devices choose $2=$ 10). For each combination, a measurement trial was performed, operating each device separately and both devices simultaneously. Measurement trials are approximately 24 seconds in length, consisting of three 8-second segments.

Figure 4.3 illustrates an example of one measurement trial for device ' $A$ ' and device ' $\mathrm{B}$ ', which were electric kettle and coffee maker, respectively. For the first 8-second segment, only device ' $A$ ' is $O N$ (device 'B' is OFF). For the second 8-second segment, both device 'A' and device ' $B$ ' are on. For the third 8 -second segment, only device 'B' is ON (device 'A' is OFF). Two trials for each two-device combination were performed. Therefore a total of 20 measurement trials were completed ( 10 combinations $\times 2$ trials). 


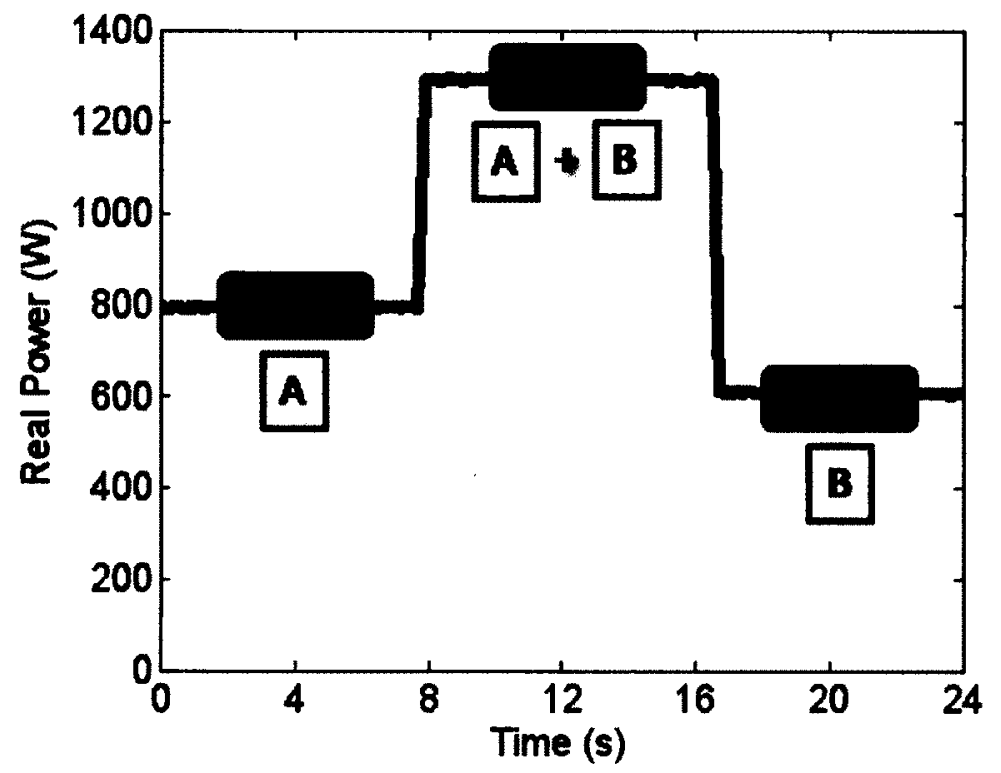

Figure 4.3: Real power versus time from an example measurement trial involving two electrical devices: device ' $A$ ' and device ' $B$ '. In this plot, the devices were electric kettle and coffee maker, respectively.

\subsubsection{Feature Extraction}

Each 24-second measurement trial was broken down into non-overlapping $100 \mathrm{~ms}$ analysis windows. The real power value was computed from the current and voltage measurements in each analysis window. To avoid the transient parts of the waveform, we only use the middle segments of the composite real power waveform, which are the steady-state portions. As depicted in Figure 4.3, 50 analysis windows ( 5 seconds in length) from the middle of each segment (shown by grey rectangular) are extracted as real power steady-state features. 


\subsubsection{Pattern Recognition}

\section{Training Set}

Training data were selected from the measurement trials, when only one device was in operation. Fifty analysis windows from the middle of first segment and third segment of each measurement trial were used for training data (shaded area of the first segment and 'third segment in Figure 4.3). For each device, there were 400 analysis windows (50 analysis windows $\times 2$ trials $\times 4$ combinations) in the training set (2000 analysis windows for all devices).

\section{Testing Set}

Testing data were selected from the measurement trials, when two devices were in operation (composite load signature). Fifty analysis windows from the middle of second segment of each measurement trial were used for testing data (shaded area of the second segment in Figure 4.3). The extracted features from these analysis windows correspond to the simultaneous operation of device ' $A$ ' and device ' $B$ '. Testing data for device ' $A$ ' were computed by subtracting the mean feature value of device ' $B$ ' taken from the third segment of the measurement trial. Testing data for device ' $B$ ' were computed by subtracting the mean feature value of device ' $A$ ' taken from the first segment of the measurement trial. For each device, there were 
400 analysis windows in the testing set (2000 analysis windows for all devices).

\section{Pattern Classification}

Classification was simply performed using Mahalanobis discriminant analysis [74].

\subsubsection{Results}

Table 4.5 lists the mean real power ( \pm one standard deviation) for the electrical devices, when operated individually and when operated in pairs. Diagonal elements in Table 4.5 contain the mean real power for the five individual electrical devices (data from analysis windows from the first and third 8-second segment of each measurement trial). For example, entry $(1,1)$ of Table 4.5 is the mean real power for load 1 (computer LCD monitor). Entry $(2,2)$ of Table 4.5 is the mean real power for load 2 (electric kettle). The off-diagonal elements contain the mean real power for the various combinations of two devices (data from analysis windows from the second 8-second segment of each measurement trial). The rows indicate the first device that is ON and the columns indicate the second device that is on. For example, entry $(1,2)$ of Table 4.5 is the mean real power when load 1 and load 2 are ON simultaneously. As real power meets the feature-additive criterion, the summation of entries $(1,1)$ and $(2,2)(28.51 \mathrm{~W}+798.49 \mathrm{~W}=$ $827.00 \mathrm{~W})$ should equal entry $(1,2)(822.74 \mathrm{~W})$. The lower triangular part of 
Table 4.5 is empty because of symmetry (e.g., entry $(2,1)$ would be the mean real power when load 2 and load 1 are ON simultaneously, which is the same as entry $(1,2))$.

Table 4.5: Mean real power ( \pm one standard deviation) for the electrical devices, when operated individually and when operated in pairs.

\begin{tabular}{|c|c|c|c|c|c|}
\hline & Load 1 & Load 2 & Load 3 & Load 4 & Load 5 \\
\hline Load 1 & $28.51 \pm 0.75$ & $822.74 \pm 1.97$ & $638.38 \pm 1.83$ & $87.32 \pm 0.69$ & $41.13 \pm 0.94$ \\
\cline { 1 - 4 } Load 2 & & $798.49 \pm 9.97$ & $1288.01 \pm 2.52$ & $859.75 \pm 15.59$ & $804.25 \pm 5.01$ \\
\hline Load 3 & & & $608.42 \pm 2.50$ & $659.81 \pm 1.43$ & $623.56 \pm 2.95$ \\
\hline Load 4 & & & & $59.48 \pm 0.76$ & $71.11 \pm 0.38$ \\
\hline Load 5 & & & & $13.31 \pm 0.63$ \\
\hline
\end{tabular}

Table 4.6 shows the classification confusion matrix (results are for the testing data only; i.e., classification results for the training data are not included in the confusion matrix). When using real power as a unique feature to disaggregate the multiple operation of two electrical devices, the classification accuracy of $95 \%$ is achieved.

Table 4.6: Classification confusion matrix for real power (Mean classification accuracy 95\%).

\begin{tabular}{|c|c|c|c|c|c|c|}
\hline & \multicolumn{5}{|c|}{ Predicted load } \\
\hline & & Load 1 & Load 2 & Load 3 & Load 4 & Load 5 \\
\hline \multirow{5}{*}{$\frac{\text { go }}{\frac{8}{0}}$} & Load 1 & 400 & 0 & 0 & 0 & 0 \\
\hline & Load 2 & 0 & 400 & 0 & 0 & 0 \\
\hline & Load 3 & 0 & 100 & 300 & 0 & 0 \\
\hline & Load 4 & 0 & 0 & 0 & 400 & 0 \\
\hline & Load 5 & 0 & 0 & 0 & 0 & 400 \\
\hline
\end{tabular}




\subsubsection{Discussion}

High classification accuracy $(95 \%)$ is achieved using the real power. This is not unexpected given the high repeatability of the real power measurements, indicated by the low standard deviation values in Table 4.5. Table 4.6 indicates that load 3 (coffee maker) is the only electrical device that is misclassified. Load 3 is misclassified as load 2 (electric kettle) $25 \%$ of the time. Load 2 and load 3 have comparable real power values (798.49 W and $608.42 \mathrm{~W}$, respectively), which are much higher than the other devices; as well these devices the highest standard deviation values (Table 4.5).

Closer examination of the classification results reveal the misclassification corresponds to the measurement trials when load 2 and load 3 are ON simultaneously. Examining Table 4.5, it is noted that the real power when both load 2 and load 3 are ON simultaneously is $1288.01 \mathrm{~W}$, whereas the summation of the real power of these individual devices is $1406.91 \mathrm{~W}$.

To investigate this difference, we employed an electricity usage monitor (Kill-A-Watt, P3 International, New York NY, USA) [77]. The electricity usage monitor was used to measure load 2 (electric kettle) by inserting it between the power bar and the load 2 device. When load 2 was operating individually, the real power measured by the Kill-A-Watt monitor was $834 \mathrm{~W}$. When load 3 (coffee maker) was then turned on, the real power consumption of load 2 was dropped to $767 \mathrm{~W}$. It showed that when load 2 
was operating simultaneously with load 3 , the real power measurement was $67 \mathrm{~W}$ lower than when load 2 was operating by itself. When these two relatively high powered devices are simultaneously in operation, their power consumptions ends up being lower than when the devices are operated individually. It is hypothesized that the total electrical supply is limited (e.g., current limited). In addition, these devices (electric kettle and coffee maker) probably do not regulate their power supply. Therefore, while real power meets the feature-additive criterion theoretically, we may not observe this practically.

\subsection{Load Identification during the Simultaneous Operation of five Electrical Devices}

In this section, our prototype NILM system has been demonstrated for the recognition of five electrical devices when operating simultaneously. A NILM system (Figure 4.4) was constructed to monitor multiple devices in operation simultaneously, along with feature extraction, event detection, and pattern classification methods to disaggregate the individual electrical devices from the composite signal. Real power is used in this work as a unique load signature for load identification. Experimental results of this work showed 
that the proposed approach can achieve $86.25 \%$ device recognition accuracy for five electrical devices operating simultaneously.

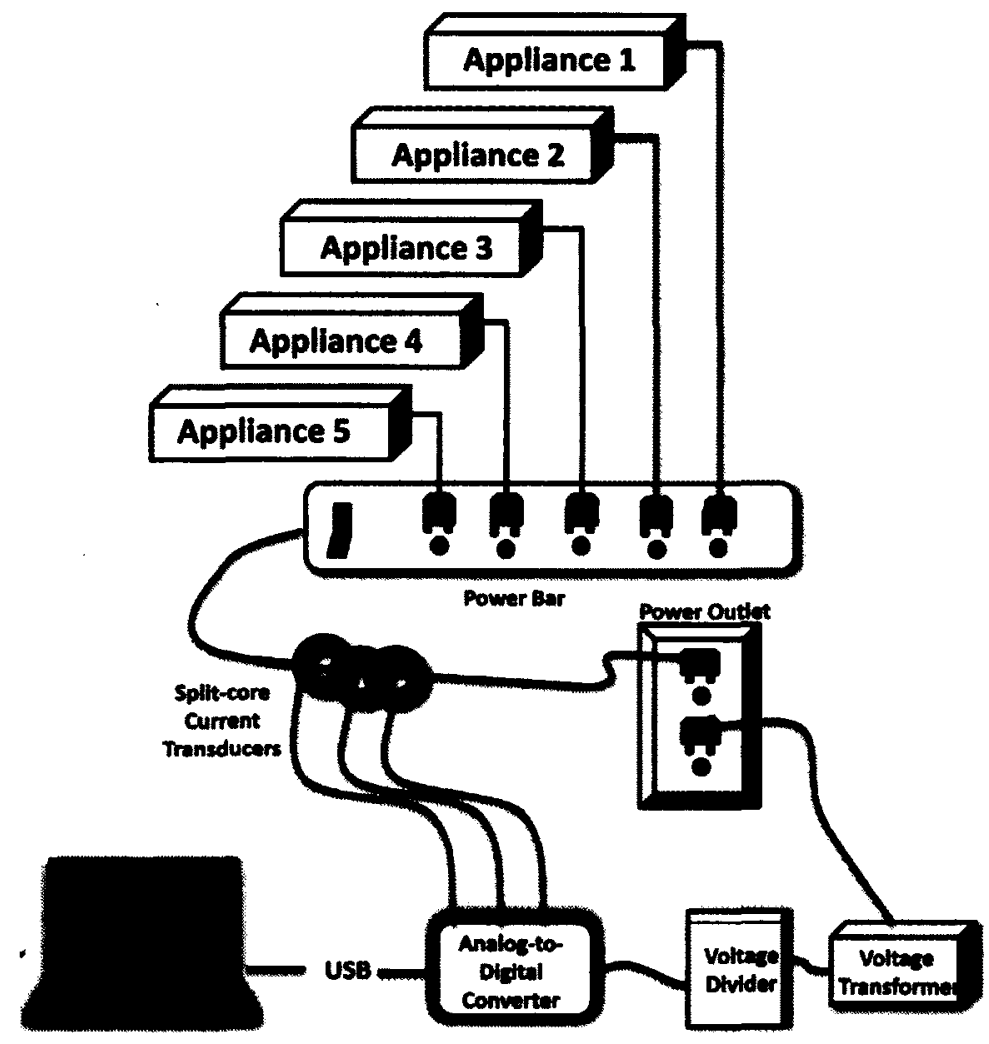

Figure 4.4: Experimental setup for our NILM prototype (load identification during the simultaneous operation of five electrical devices).

\subsubsection{Data Acquisition}

Five household electrical devices (the same as section 4.2) were used as loads in this section: electric kettle (Load 1), coffee maker (Load 2), computer LCD monitor (Load 3), incandescent lamp (Load 4), and fluorescent lamp (Load "5). In this section, we are interested in 
disaggregating the composite load signal (multiple operation of five electrical devices) into its components, which are the individual electrical devices. We extend the method we used in section 4.2 for disaggregation of all five electrical devices operating simultaneously. Figure 4.5 illustrates an example of one measurement trial for five electrical devices; it is indicated when each load is turned on or off.

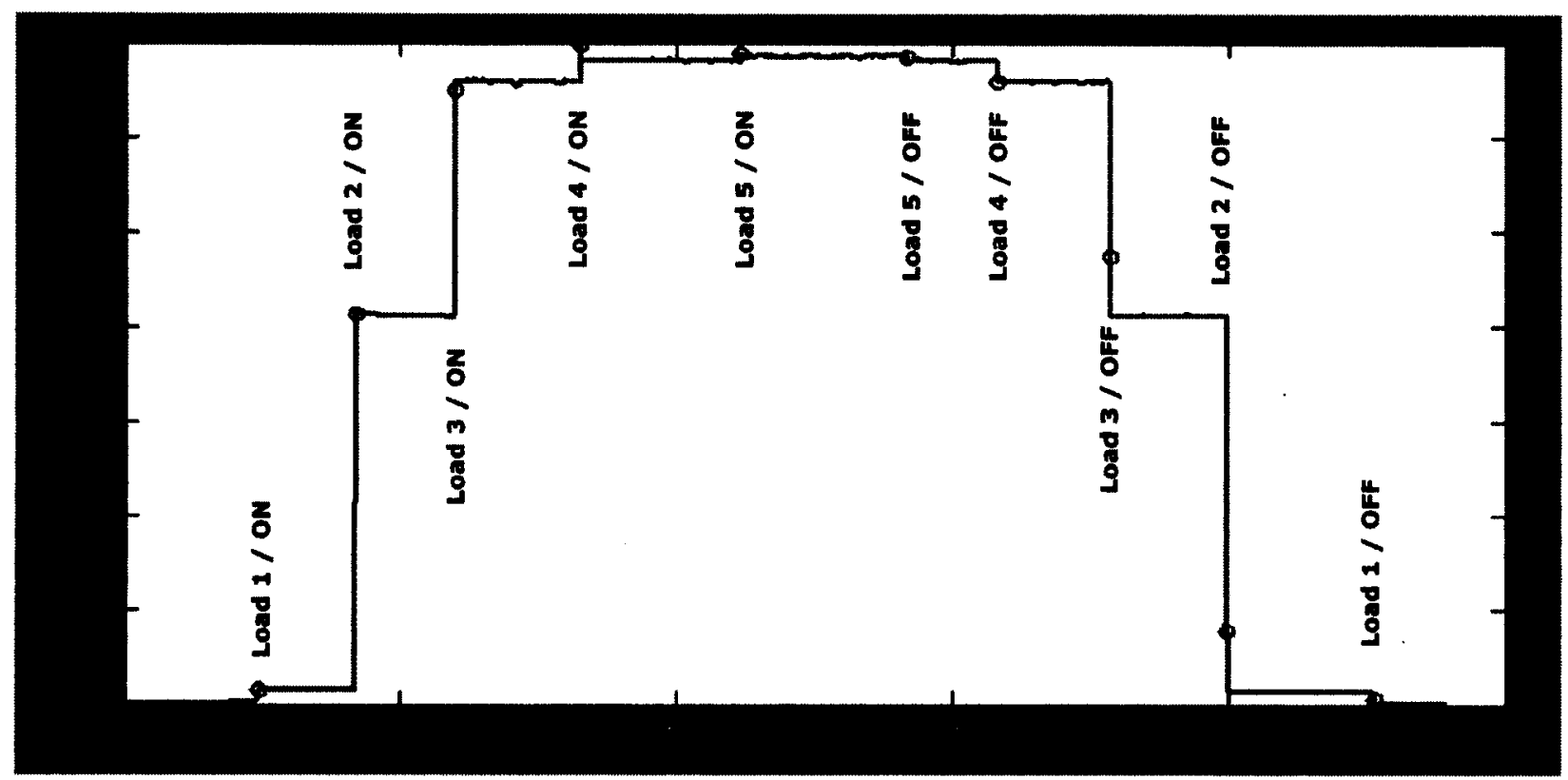

Figure 4.5: An example of real power waveform for one of the measurement trials for the simultaneous operation of multiple electrical devices. The consequence of turning on and turning off of the 5 electrical devices is shown on step-changes.

A total of 8 measurement trials were completed with each measurement lasting approximately 65 seconds. Table 4.7 shows operation of the five electrical devices during each measurement trial (ON/OFF represents the turning on/off of each individual electrical device). For instance, the first row 
of table 4.7 represents that Load 1 is the first device which is turned on. Then, Load 5 is turned on while the Load 1 is operating simultaneously. Load 3 , load 4, and load 2 are turned on respectively. Subsequently, Load 2, load 4 , load 3, and load 5 are turned off. Load 1 is the last device which is turned off. Figure 4.5 corresponds to the measurement trial number 8 (see Table 4.7). Real power waveforms of all the 8 measurement trials are presented in appendix $A$.

Table 4.7: Turning on and turning off sequences of five electrical devices during each measurement trial.

\begin{tabular}{|c|c|c|c|c|c|c|c|c|c|c|}
\hline Trial 1 & $1 / O N$ & $5 / O N$ & $3 / O N$ & $4 / O N$ & $2 / O N$ & 2/OFF & 4/OFF & 3/OFF & 5/OFF & $1 /$ OFF \\
\hline Trial 2 & $1 / \mathrm{ON}$ & $2 / O N$ & $4 / O N$ & $3 / \mathrm{ON}$ & 4/OFF & $5 / O N$ & 2/OFF & 5/OFF & 3/OFF & $1 /$ OFF \\
\hline Trial 3 & $1 / \mathrm{ON}$ & $2 / O N$ & $4 / O N$ & 2/OFF & $5 / O N$ & $3 / O N$ & $3 /$ OFF & 5/OFF & 4/OFF & $1 /$ OFF \\
\hline Trial 4 & $1 / \mathrm{ON}$ & $3 / \mathrm{ON}$ & $2 / O N$ & $4 / O N$ & 2/OFF & $5 / O N$ & 3/OFF & 5/OFF & 4/OFF & $1 /$ OFF \\
\hline Trial 5 & $1 / \mathrm{ON}$ & $4 / O N$ & $3 / \mathrm{ON}$ & $5 / O N$ & $2 / O N$ & $2 /$ OFF & 5/OFF & $3 /$ OFF & $4 /$ OFF & 1/OFF \\
\hline Trial 6 & $1 / \mathrm{ON}$ & $5 / O N$ & $2 / O N$ & $4 / O N$ & $3 / \mathrm{ON}$ & 4/OFF & $2 / \mathrm{OFF}$ & $3 /$ OFF & 5/OFF & $1 /$ OFF \\
\hline Trial 7 & $1 / \mathrm{ON}$ & $5 / O N$ & $2 / O N$ & 5/OFF & $3 / \mathrm{ON}$ & $4 / O N$ & $3 /$ OFF & 4/OFF & $2 / \mathrm{OFF}$ & $1 /$ OFF \\
\hline Trial 8 & $1 / \mathrm{ON}$ & $2 / O N$ & $3 / \mathrm{ON}$ & $4 / O N$ & $5 / O N$ & $5 /$ OFF & 4/OFF & $3 /$ OFF & 2/OFF & $1 /$ OFF \\
\hline
\end{tabular}

\subsubsection{Feature Extraction}

Each measurement trial (total of 8 measurement trials in this work) was broken down into non-overlapping $100 \mathrm{~ms}$ analysis windows and real power values were extracted for each measurement trial. 


\subsubsection{Pattern Recognition}

\section{Event Detection}

The event detection method explained in section 3.2.3 was used in this section to find the events (i.e., when an electrical device turns on or off) in extracted real power waveform. All 10 indices ( 5 devices $\times 2$ state change ON/OFF) were correctly identified for each measurement trial, indicating the location and time of step change in the extracted real power waveform.

\section{Training set}

The same training set we established in the second phase of our steadystate analysis (classification of two electrical devices when operating simultaneously), were used as training set (section 4.2.3). As mentioned in section 4.2.3, training data were selected from the measurement trials, when only one of the five electrical devices was in operation. Fifty analysis windows from each single electrical device operation were used for training data. For each device, there were 4 different measurement trials (200 analysis windows for an individual electrical device) in the training data and 1000 analysis windows for all five electrical devices.

\section{Testing Set}

Testing data were selected from the 8 measurement trials, when five devices were in operation (composite load signature). The output of the event detection step is collection of 10 time indices representing the two state 
changes (turning on and off) of 5 electrical devices during each measurement trial. When an event " $i$ " is detected one of the five electrical devices has turned either on or off. In order to select our testing data, the difference between the segment before and the segment after event $i$ is examined. The segment before event $i$ includes 50 analysis windows from index (i) -70 to index (i) -21 (this corresponds to 2.5 seconds before the event), while the segment after event $i$ includes 50 analysis windows from index $(i)+11$ to index $(i)+60$ (this corresponds to 2.5 seconds after the event). Testing data for each device were computed by subtracting the mean feature value of $\mathbf{5 0}$ analysis windows from the segment after event $i$ taken from the mean value of 50 analysis windows from the segment before event i. The absolute of these values indicate the magnitude of step change for each detected event in the real power waveform. The sign specifies the change state; the positive and negative sign indicate that an individual device is turned on and off, respectively. This method is applied to all of the 10 indices (events) detected in each of those 8 measurement trials. For each device, there were 16 values ( 8 measurement trials $\times 2$ state change ON/OFF) in the testing set and 80 values for all five electrical devices.

\section{Pattern Classification}

Classification was simply performed using k-nearest neighbour (KNN) classifier $(k=1)$ in order to determine which device turned on or off [74]. 
The Mahalanobis discriminant analysis was also used here for classification but higher recognition accuracies were achieved using KNN classifier. Only the KNN classification results are shown here.

\subsubsection{Results}

Table 4.8 shows the classification confusion matrix for identification of five electrical devices with the mean classification accuracy of $86.25 \%$ (results are for the testing data only; i.e., classification results for the training data are not included in the confusion matrix). Table 4.9 lists the mean real power differences for all five electrical devices for the test data. For each cell of the Table 4.9, the first value indicates the real power change of an associated with the electrical device when it turns on $(+)$ and the second value shows the turn off $(-)$ real power change.

Table 4.8: Classification confusion matrix for identification of five electrical devices using real power usage of devices (Mean classification accuracy: $86.25 \%$ ).

\begin{tabular}{|c|c|c|c|c|c|c|}
\hline & \multicolumn{5}{|c|}{ Predicted load } \\
\hline & & Load 1 & Load 2 & Load 3 & Load 4 & Load 5 \\
\hline \multirow{5}{*}{ 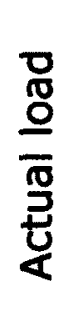 } & Load 1 & 16 & 0 & 0 & 0 & 0 \\
\hline & Load 2 & 0 & 8 & 8 & 0 & 0 \\
\hline & Load 3 & 0 & 0 & 16 & 0 & 0 \\
\hline & Load 4 & 3 & 0 & 0 & 13 & 0 \\
\hline & Load 5 & 0 & 0 & 0 & 0 & 16 \\
\hline
\end{tabular}


Table 4.9: Mean real power change of 5 electrical devices when operating simultaneously during each measurement trial. The first and second values of each cell represent the turn on and turn off real power change for each electrical device.

\begin{tabular}{c|c|c|c|c|c}
\hline \multirow{2}{*}{ Trial } & \multicolumn{5}{|c}{ Real Power (Watt) } \\
\cline { 2 - 6 } 1 & Load 1 & Load 2 & Load 3 & Load 4 & Load 5 \\
\hline \multirow{3}{*}{2} & +27.34 & +672.78 & +616.62 & +49.86 & +13.46 \\
& -27.45 & -672.58 & -610.39 & -51.73 & -13.17 \\
\hline \multirow{3}{*}{3} & +27.16 & +796.83 & +491.17 & +48.37 & +9.07 \\
& -27.08 & -681.29 & -609.27 & -40.76 & -12.09 \\
\hline \multirow{3}{*}{4} & +27.31 & +801.19 & +609.59 & +49.55 & +13.56 \\
& -27.35 & -788.47 & -603.69 & -59.04 & -13.38 \\
\hline \multirow{2}{*}{5} & +27.33 & +683.14 & +617.46 & +45.69 & +12.25 \\
& -27.34 & -677.51 & -602.95 & -59.18 & -13.61 \\
\hline \multirow{2}{*}{6} & +27.33 & +673.80 & +610.19 & +59.02 & +10.42 \\
& -27.40 & -675.21 & -604.43 & -59.30 & -12.73 \\
\hline \multirow{2}{*}{7} & +27.17 & +793.91 & +493.56 & +50.60 & +13.04 \\
& -27.05 & -680.28 & -607.89 & -44.32 & -13.11 \\
\hline \multirow{2}{*}{8} & +27.37 & +800.19 & +501.83 & +40.84 & +13.01 \\
& -27.17 & -798.46 & -489.34 & -49.83 & -11.50 \\
\hline
\end{tabular}

Figure 4.6 shows the results of load identification for two different measurement trials of simultaneously operation of five electrical devices. Figure 4.6 (a) shows the measurement trial number 2 with the recognition accuracy of $80 \%$, when Load 2 (electric kettle) is misclassified as Load 3 (coffee maker) and Load 4 (incandescent lamp) is misclassified as Load 1 (computer LCD monitor). Figure 4.6 (b) shows the measurement trial number 3 with the recognition accuracy of $100 \%$ when turning on and off of all five electrical devices is recognized truly. In the Figure 4.6, for each step- 
change of the real power waveforms (green circles), the names of the electrical devices are indicated; the first electrical device represents the name of actual electrical device which changes state on that time while the electrical devices written inside the brackets represents the results of our load identification method.

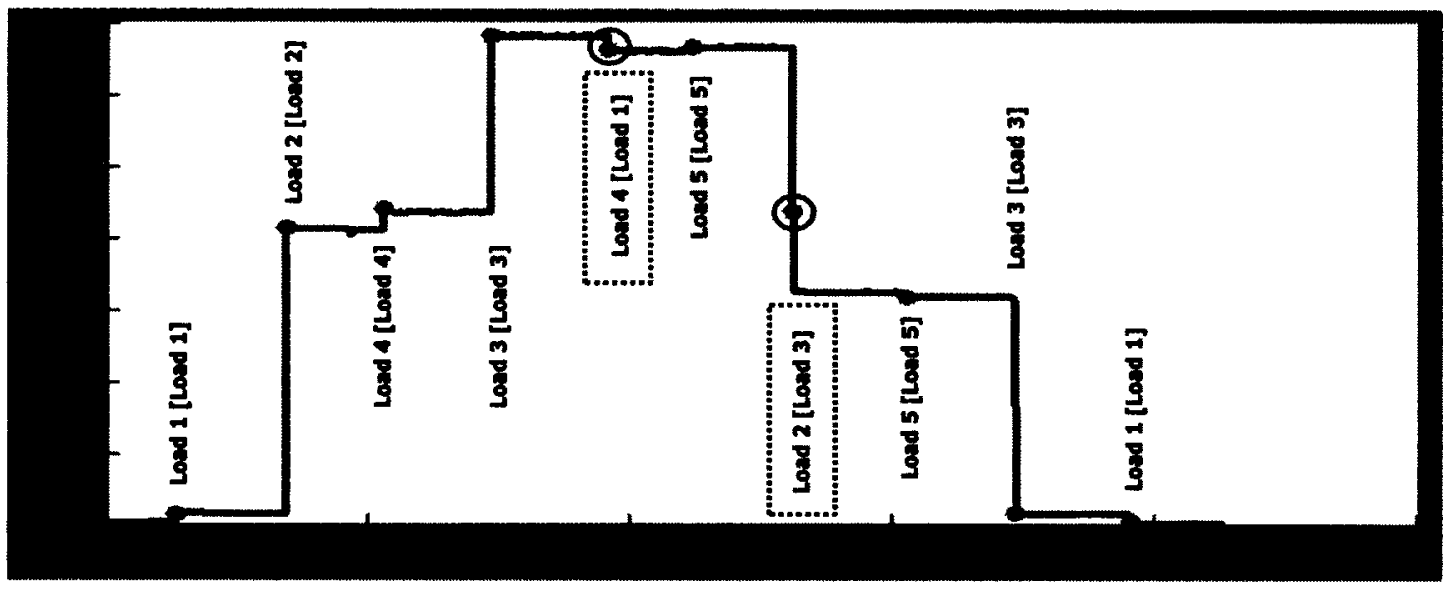

(a)

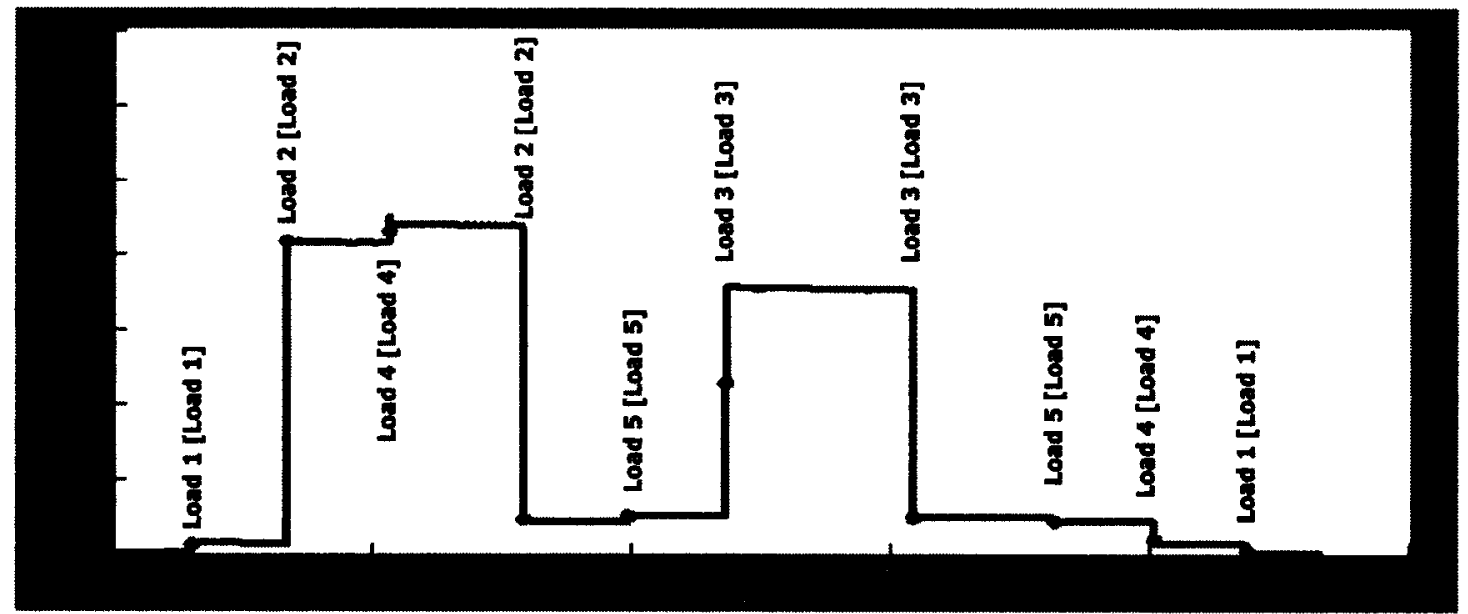

(b)

Figure 4.6: Real power consumption during the multiple operation of five electrical for (a) trial 2 (load identification accuracy 80\%) and (b) trial 3 (load identification accuracy $100 \%$ ). Green circles are used to indicate the detect events. Red circles show the events in which loads are misclassified. Electrical devices that are turned on or off are indicated, with the classification result in square brackets. 


\subsubsection{Discussion}

High load recognition accuracy $(86.25 \%)$ is achieved using the real power as a unique feature for load disaggregation (see Table 4.8). This is not unexpected given the values of the real power measurements of each individual electrical device during 8 different measurement trials, indicated in Table 4.9.

Table 4.8 indicates that Load 2 (electrical kettle) and Load 4 (Incandescent lamp) are the electrical devices that are misclassified most frequently. Load 2 is misclassified as load 3 (coffee maker) $50 \%$ of the time. As shown in Table 4.9, Load 2 and load 3 have comparable real power values (Load 2: electric kettle and Load 3: coffee maker) during simultaneous operations, which are much higher than the other devices. Closer examination of classification results of these two real power waveforms reveal that all the misclassifications of load 2 as load 3 corresponds to the measurement trials when load 3 is already ON and load 2 is either switched on or off (i.e., trials $1,2,4,5$, and 6). This issue is similar to what was explained in section 4.2.5. Our investigation showed that when these two relatively high powered devices (Load 2 and Load 3) are simultaneously in operation, their power consumptions ends up being lower than when the devices are operated individually. The result of load identification during the multiple operations of five electrical devices is consistent with our previous hypothesis that the total electrical supply is 
limited and these devices (electric kettle and coffee maker) probably do not regulate their power supply. As stated in Section 3.2.1, while real power meets the feature-additive criterion theoretically, we may not observe this practically.

\subsection{Conclusions}

In our work, A NILM system has been successfully demonstrated for the recognition of electrical devices using their steady state power consumption characteristics (real power and reactive power). In this chapter we have demonstrated this for electrical devices operated individually and when operated simultaneously. The proposed method in this chapter worked with high device recognition accuracy, demonstrating that real power and reactive power are useful features to identify electrical devices and can serve as effective complementary features to one another. Moreover, this work showed that when relatively high powered devices are simultaneously in operation, their power consumptions ends up being lower than when the devices are operated individually. Therefore, while real power meets the feature-additive criterion theoretically, we may not observe this practically. 


\section{Transient Analysis}

This chapter describes our methodology for the NILM systems based on the switching transient behaviour of electrical devices. Section 5.1 presents our transient based NILM method including data acquisition, feature extraction, normalization, and pattern recognition. In section 5.2, the results of our experimental tests and discussions are presented. Finally, the conclusions for the transient signature based experiments are presented in Section 5.3.

\subsection{Methods}

Similar to steady-state analysis in Chapter 3, a NILM system (Figure 5.1) was constructed to monitor the usage of four electrical devices connected to a common power bar. Our prototype NILM system monitors current and 
voltage signals at the input of the power bar to simulate the NILM systems capability to monitor at a single electrical source. Experimental testing of our NILM prototype in this section is performed using four common household electrical devices, where only one device was $\mathrm{ON}$ at a time. Our experimental results for single electrical device recognition of four devices showed that our proposed approach can achieve $80.83 \%$ classification accuracy.

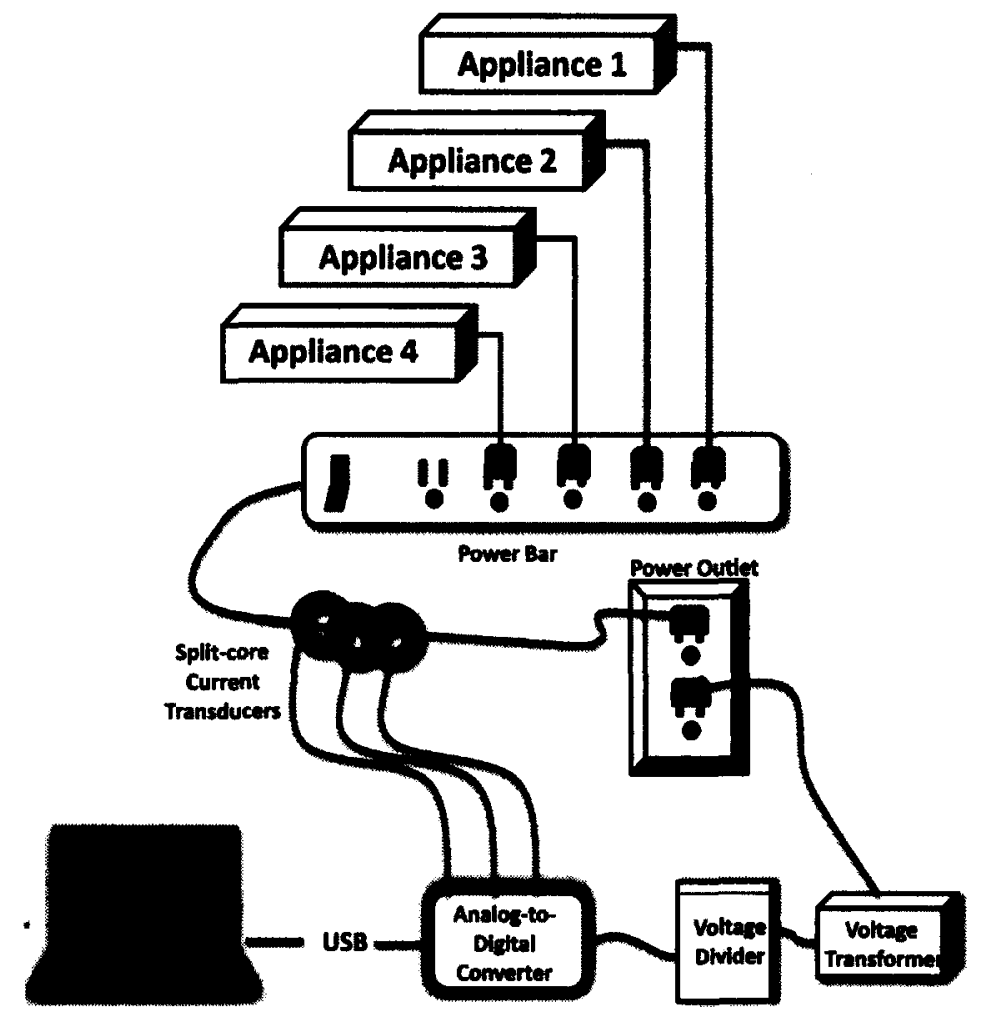

Figure 5.1: Experimental setup for our prototype NILM system for classification of single electrical devices based on their transient profiles.

Switching transient is defined as the electrical behaviour of an electrical device when it is either switched on or off. Each electrical device contains unique features in its transient behaviour; so that electrical devices can be 
identified based on their observable transient profiles (e.g., real power or reactive power waveforms) [27][36]. Transient characteristics are intimately related to the task that the electrical device performs [40]. For example, the turn on transient profile of a computer CRT monitor and kitchen blender are distinct because the motor in a kitchen blender is fundamentally different from the display electronics in a computer CRT monitor. It is stated in [36] that most electrical devices possess repeatable transient profiles or at least sections of their transient profiles are repeatable.

As mentioned in Chapter 4, NILM systems are not able to detect the usage of continuously variable loads based on their steady-state features. However, distinctive transient profiles tend to persist in continuously variable loads such as Variable Speed Drivers (VSDs) which makes it possible to monitor them non-intrusively by tracking the transient behaviours during their operation.

Since, the real power values are closely related to an electrical device's operational quantities, they are used as reliable indicators for switching on transient waveforms [12]. Figure 5.2 shows the measured real power waveform during the turn on transient of a) computer CRT monitor; b) kitchen blender; and c) hair dryer. In each case, the switching on transient waveform is observed to exhibit patterns created by the unique electrical, mechanical, and thermal processes that occur in each class of electrical devices during the turn on event. 


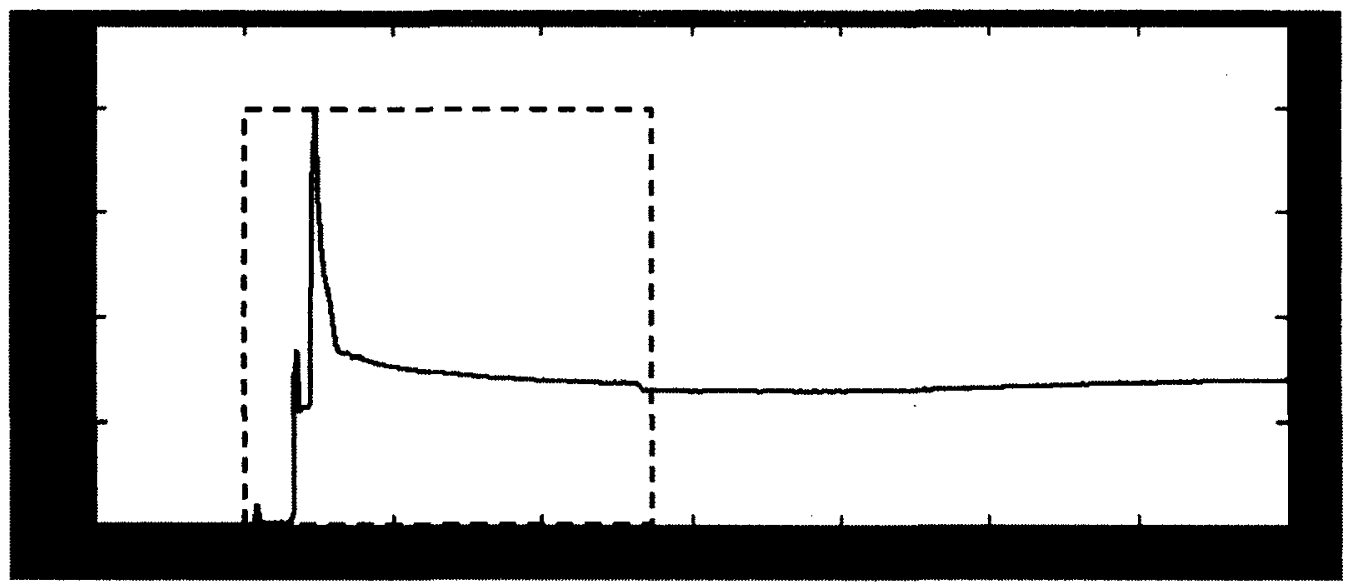

(a)

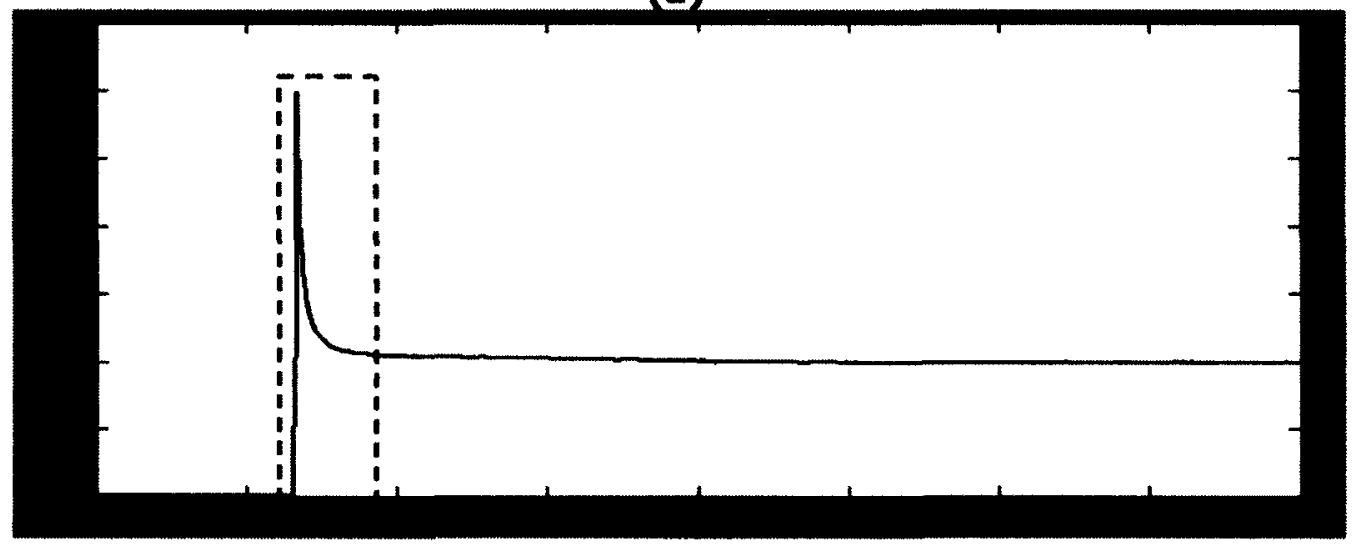

(b)

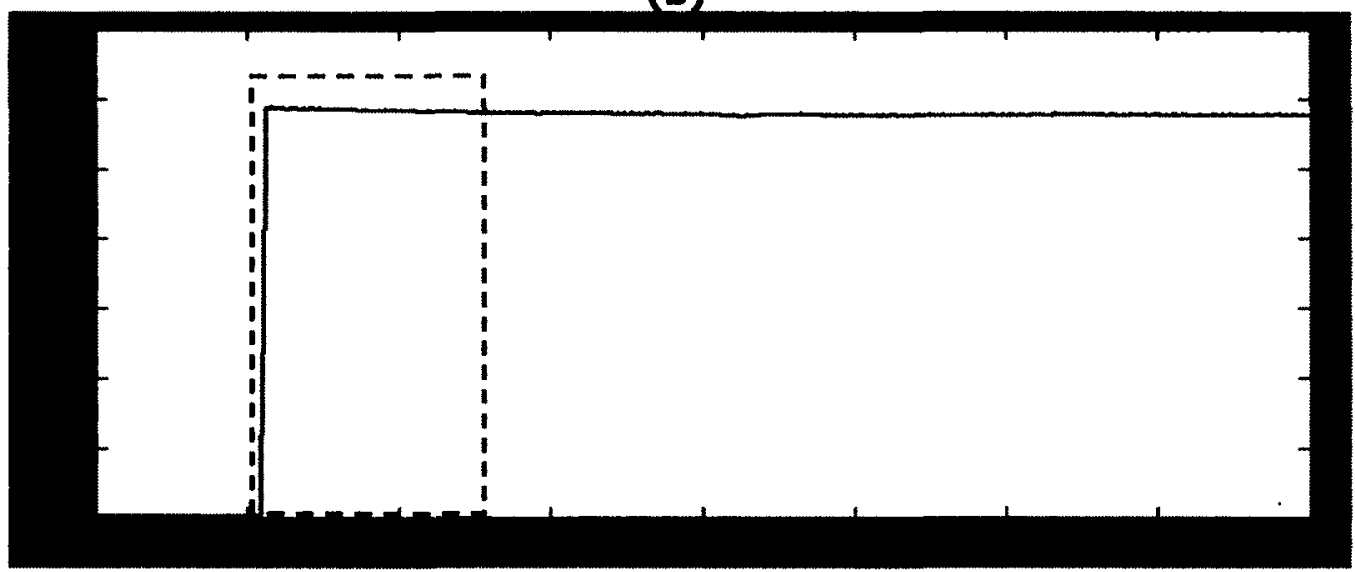

(c)

Figure 5.2: Switching on transient profile of three different electrical devices (real power values versus time): (a) computer CRT monitor, (b) kitchen blender, and (c) Hair dryer. 


\subsubsection{Data Acquisition}

Four household electrical devices were used as loads in this section: kitchen blender (Load 1), computer CRT monitor (Load 2), air heater (Load 3), and hair dryer (Load 4). The electrical devices used for the transient analysis are different from the steady state analysis devices. Electrical devices used for transient analysis have motors, except the CRT monitor which has a flyback transformer. These electrical components in the devices were expected to be associated with long duration transient behaviours. In this section, we are interested in the recognition of electrical devices when they are operating individually (i.e., electrical devices were not operated simultaneously). A sampling frequency of $20 \mathrm{kHz}$ is used in this work to capture transient signatures of electrical devices when they are switching on. Figure 5.3 illustrates the RMS current, RMS voltage, and real power waveforms of an example measurement trial for load 2 (computer CRT monitor). For the first segment (10-second), load 2 is OFF (although the load was plugged in the power bar). For the second segment, load 2 is turned on and run for a period of 40 seconds. For the third segment (10-second), load 2 is OFF. Data were collected for each device for 10 trials, with each measurement lasting 60 seconds. A total of 40 measurements were completed (4 electrical devices $\times 10$ trials). 


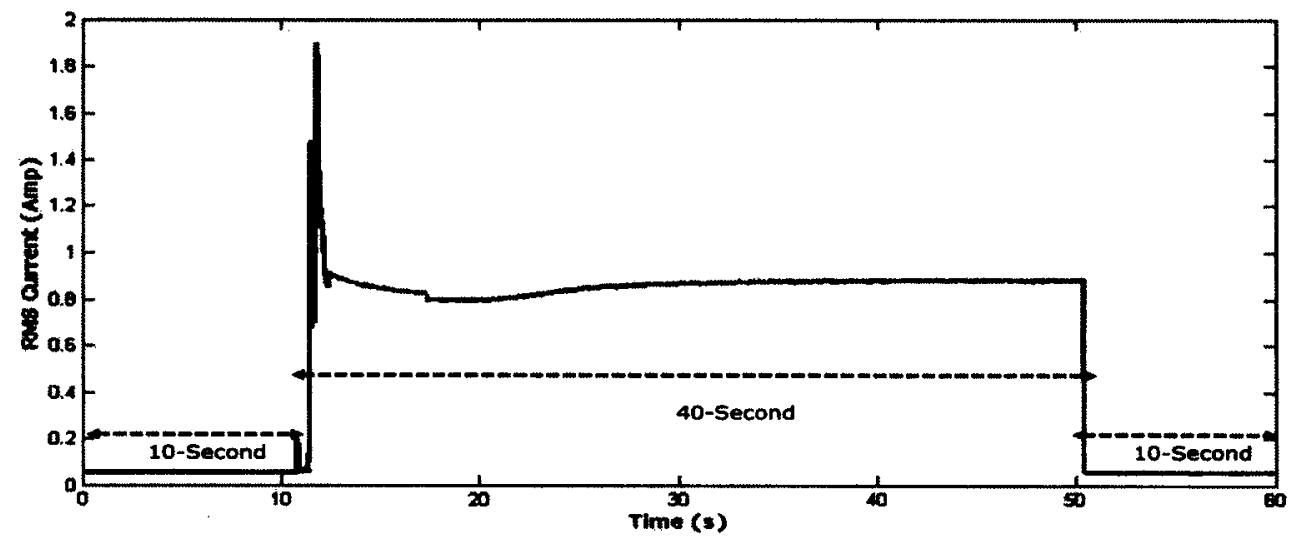

(a)

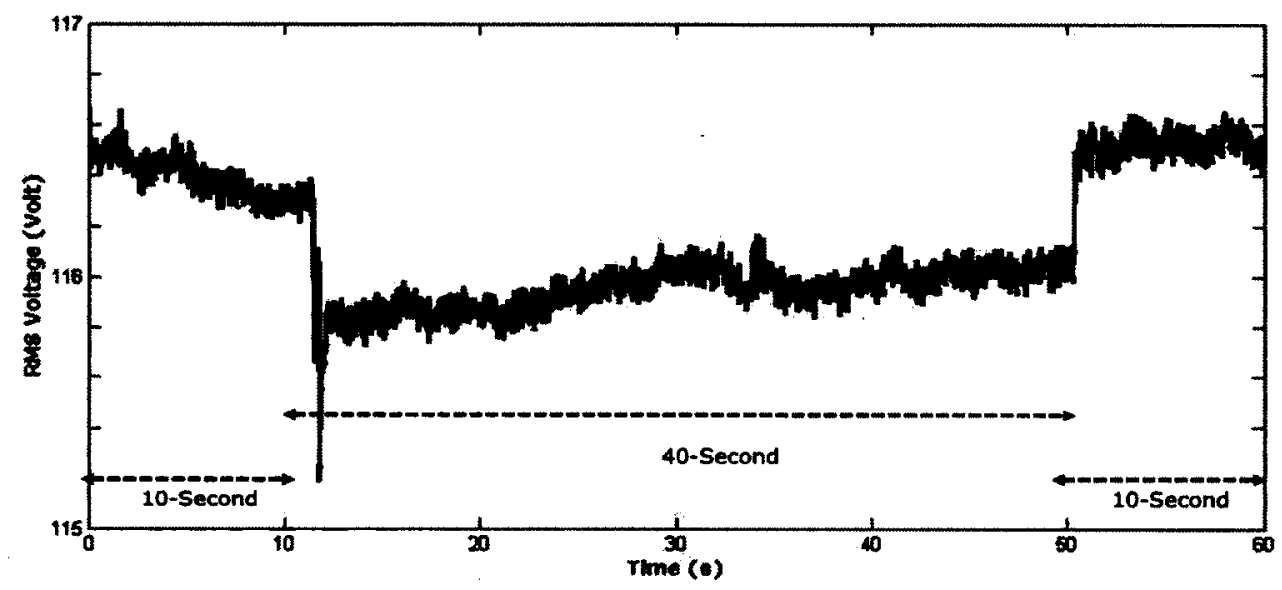

(b)

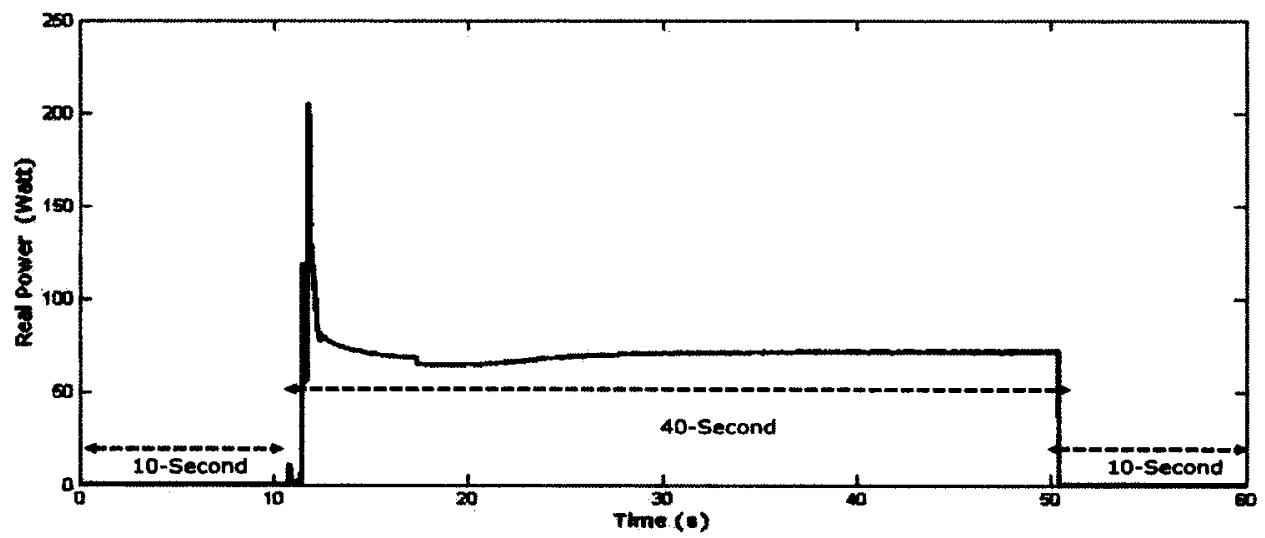

(c)

Figure 5.3: (a) RMS Current versus time, (b) RMS Voltage versus time, and (c) Real power versus time from an example measurement trial for load 2. 


\subsubsection{Feature Extraction}

In this section, features are computed from sliding analysis window [72]. An example of the sliding window is depicted in Figure 5.4 , shown with analysis windows of $50 \mathrm{~ms}$ in length, spaced $1 \mathrm{~ms}$ apart. A single feature vector (i.e. real power) is produced from each analysis window. Each 60-second measurement trial was broken down into overlapping $50 \mathrm{~ms}$ analysis windows (59951 analysis windows per measurement). The real power value was computed from the current and voltage measurements in each analysis window.

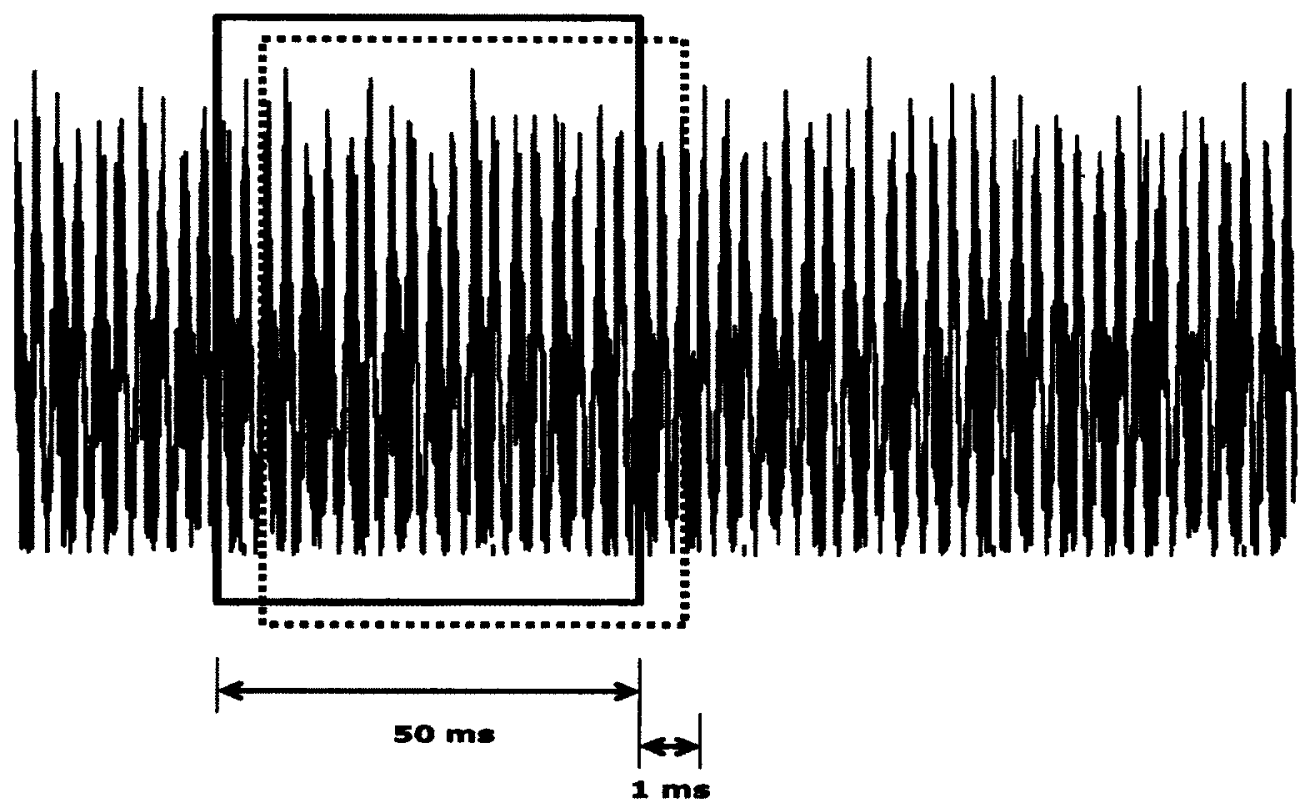

Flgure 5.4: An example of sliding analysis window for feature extraction (based on [72]).

\subsubsection{Event Detection}

The real power is input to an event detection algorithm which finds the times of all step-like changes. The event detection method explained in section 
3.2.3 was used in this section to find the events in extracted real power waveform. Two indices were correctly identified for each measurement, indicating the location and time of step change in the extracted real power waveform (i.e., when an individual electrical device is turned on and off). A total of 80 indices were identified for all measurement trials ( 4 devices $\times 10$ trials $\times 2$ state change ON/OFF).

\subsubsection{Normalization}

In the transient analysis, we aim to classify the electrical devices based on their switching on transient waveforms. To eliminate the contributions of steady-state information, we normalize the recorded real power waveforms, such that steady-state real power is unity. As a result classification accuracy is based solely on the transient portion of real power waveforms. During this step, our system selects 2 windows of samples from each measurement trial which will be used for normalization. Figure 5.5 illustrates an example of one measurement trial for load 2 (computer CRT monitor) along with two selected segments A and B (red areas). Green circles of Figure 5.5 show the switch on/off events as the outputs of our event detection method which determines the switching on index (index_on) and switching off index (index_off). 4000 analysis windows (the segment B in Figure 5.5) were selected from the segment before turning off index (from index_off - 4499 to index_off -500 corresponds to 4.5 seconds) to calculate the average 
steady-state real power value for each measurement trial. 15000 analysis windows were selected from segment A in Figure 5.5 (from index_on - 2500 to index_on +12499 corresponds to 15 seconds) for extracting normalized switching on transient waveforms. In order to establish normalized switching on transient waveforms, the transient real power values from segment $A$ are divided by the average real power value extracted from the steady-state operation (segment B). A total of 40 normalized switching on transient waveforms were completed ( 4 devices $\times 10$ trials). Each normalized switching on transient waveform for an individual electrical device is consisted of $15^{\prime} 000$ analysis windows.

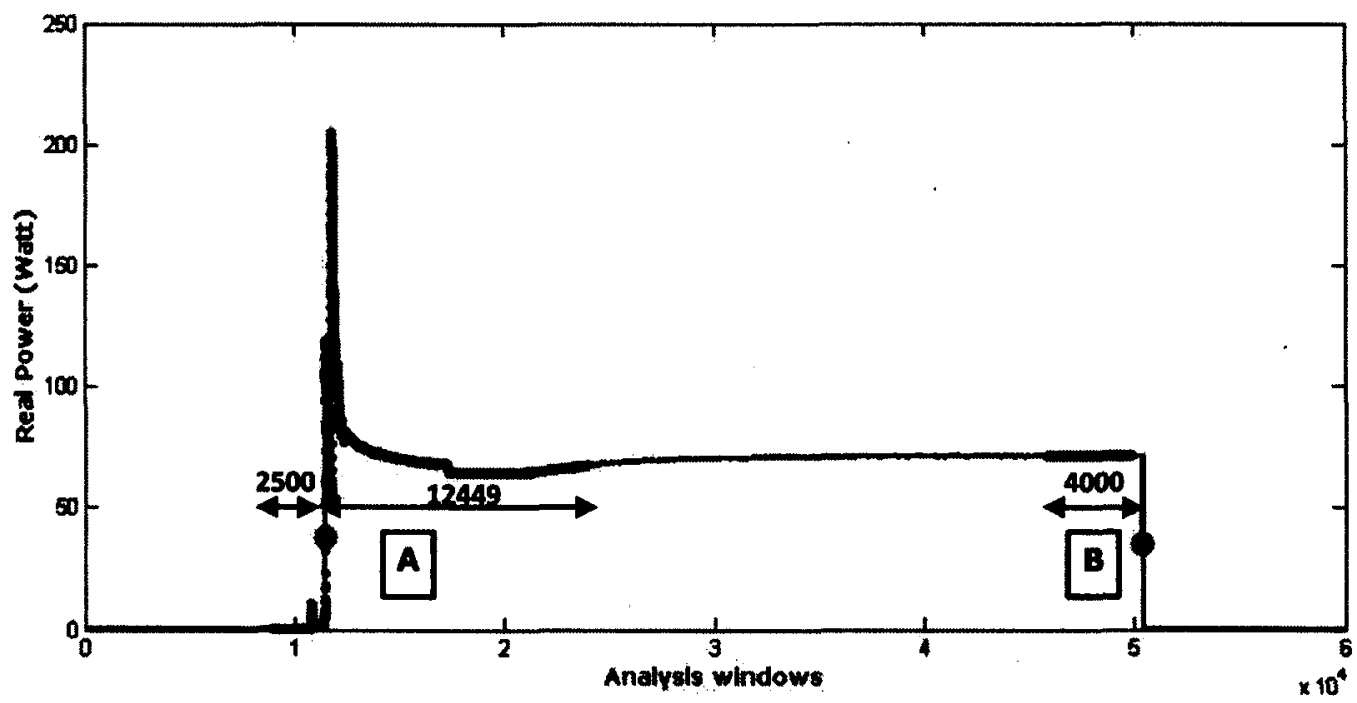

Figure 5.5: Real power versus time from an example measurement trial showing the switching on/off transient waveform of load 2 (computer CRT monitor). Green circles show the switch on/off events; the first segment (red area) shown as segment $A$ is normalized and used as switching on transient waveform while the segment $B$ is used to calculate the steady-state real power value. 


\subsubsection{Pattern Recognition}

As mentioned before, a total of 10 different trials were completed for each electrical device. Classifier training was performed using 1 trial of each device and classifier testing was performed using the remaining 9 trials. Cross-validation was performed by repeating the training and testing 10 times, such that each trial was used for training. There were 90 test cases for each device (10 repetitions $\times 9$ test trials) and a total of 360 test cases for all devices (10 repetitions $\times 9$ test trials $\times 4$ devices). Classification is simply performed using cross correlation and correlation coefficients [79][80]. In this work, cross correlation analysis is used to investigate the sequence similarity between two obtained windows of normalized switching on transient waveforms. Once, one trial (including normalized switching on transient waveforms of all four electrical devices) is selected as training set and the remaining trials were used as testing sets, cross correlation is used to determine the value of time shift. Then, the normalized switching on transient waveform of the training set is aligned according to the measured displacement values acquired from cross correlation analysis. Finally, the correlation coefficient between the aligned signal and the switching on transient waveforms of testing set is used to find the most similar transient profiles between four electrical devices. 


\subsection{Results and Discussion}

\subsubsection{Results}

Figure 5.6 shows the switching on transient waveform shapes for each electrical device during all 10 measurement trials. The switching on waveforms for each trial of a device overlaps one another, which is indicative of repeatability of switching on transient waveforms.
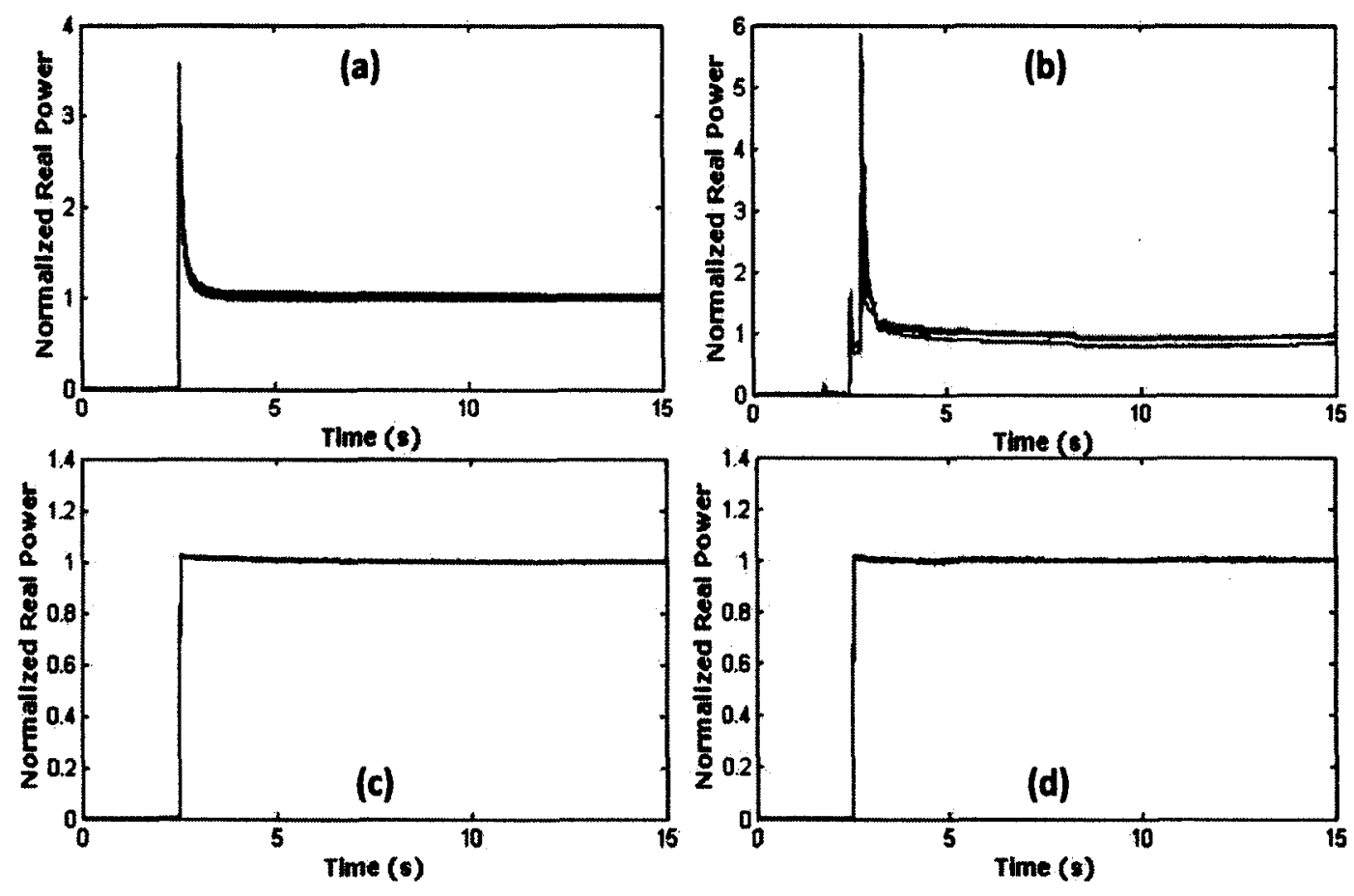

Figure 5.6: Switching on transient waveforms (measured normalized real power) during 10 different measurement trials for each individual electrical device: (a) kitchen blender, (b) computer CRT monitor, (c) air heater and, (d) hair dryer.

Table 5.1 shows the classification confusion matrix. When using normalized switching on transient waveforms to identify electrical devices, a mean classification accuracy of $\mathbf{8 0 . 8 3 \%}$ is achieved. 
Table 5.1: Classification confusion matrix for load identification (load 1: kitchen blender, load 2: computer CRT monitor, load 3: air heater, and load 4: hair dryer) based on the normalized switching on transient waveforms (Mean classification accuracy $\mathbf{8 0 . 8 3 \%}$ ).

\begin{tabular}{|c|c|c|c|c|c|}
\hline & \multicolumn{4}{|c|}{ Predicted load } \\
\hline & & Load 1 & Load 2 & Load 3 & Load 4 \\
\hline \multirow{4}{*}{ 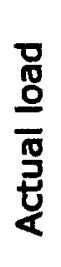 } & Load 1 & 90 & 0 & 0 & 0 \\
\hline & Load 2 & 6 & 82 & 2 & 0 \\
\hline & Load 3 & 0 & 0 & 69 & 21 \\
\hline & Load 4 & 0 & 0 & 40 & 50 \\
\hline
\end{tabular}

Table 5.2 shows the load recognition accuracy for each trial as training set. For instance, the third column of this table shows that when trial 3 was used as training set and the remaining 9 trials (trial 1 to trial 10 , except trial 3 ) were used as training sets, the recognition accuracy of $77.78 \%$ was achieved. Results show that the maximum load identification accuracy $(91.67 \%)$ is achieved when Trial 7 is used for classifier training. Furthermore, the minimum load identification accuracy $(66.67 \%)$ is achieved when Trial 9 is used for classifier training.

Table 5.2: load recognition accuracy for each measurement trial as training set.

\begin{tabular}{c|c|c|c|c|c|c|c|c|c|c}
\hline $\begin{array}{c}\text { Training } \\
\text { Set }\end{array}$ & $\begin{array}{c}\text { Trial } \\
1\end{array}$ & $\begin{array}{c}\text { Trial } \\
2\end{array}$ & $\begin{array}{c}\text { Trial } \\
3\end{array}$ & $\begin{array}{c}\text { Trial } \\
4\end{array}$ & $\begin{array}{c}\text { Trial } \\
5\end{array}$ & $\begin{array}{c}\text { Trial } \\
6\end{array}$ & $\begin{array}{c}\text { Trial } \\
7\end{array}$ & $\begin{array}{c}\text { Trial } \\
8\end{array}$ & $\begin{array}{c}\text { Trial } \\
9\end{array}$ & $\begin{array}{c}\text { Trial } \\
10\end{array}$ \\
\hline $\begin{array}{c}\text { Recognition } \\
\text { Accuracy } \\
(\%)\end{array}$ & 83.33 & 75.00 & 77.78 & 88.89 & 80.56 & 77.78 & 91.67 & 83.33 & 66.67 & 83.33 \\
\hline
\end{tabular}




\subsubsection{Discussion}

Table 5.1 indicates that load 1 (kitchen blender) and load 2 (computer CRT monitor) are classified with $100 \%$ and $91.11 \%$ recognition accuracy, respectively. Load 2 is misclassified as load $16.67 \%$ of the time. Figure 5.7 shows the closer observation of the switching on transient waveforms of load 2 during 10 different measurement trials.

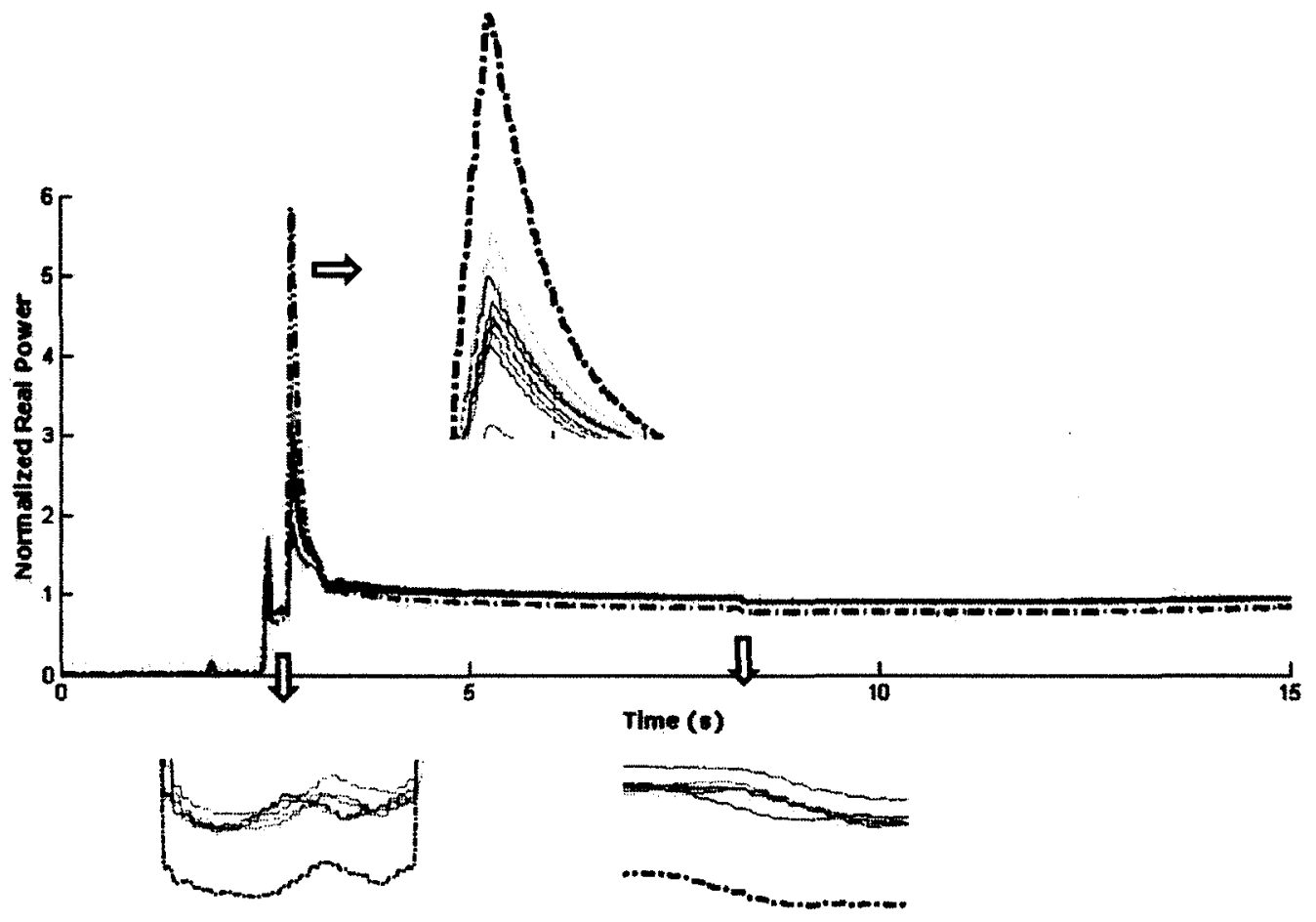

Figure 5.7: Normalized real power versus time during 10 different measurement trials to record the switching on transient waveform of load 2 . The black dashed line indicates the transient behaviour of load 2 during measurement trial 9 . Three different areas are maximized to show the difference between the switching on transient waveform of load 2 during trial 9 and the remaining trials.

Figure 5.7 reveals that misclassification of load 2 corresponds to the shape of switching on transient waveform of this load during measurement trial number 9. The black dashed line in Figure 5.7 corresponds to the 
transient behaviour of load 2 during measurement trial number 9 . The difference between the shape of load 2 during trial 10 and the remaining 9 trials makes load 2 to be misclassified as load 1. Table 5.2 confirms this misclassification; when classifier training was performed using trial number 9 and classifier testing was performed using the remaining 9 trials, the minimum recognition accuracy of $66.67 \%$ was achieved.

Table 5.1 indicates that Load 3 (air heater) and Load 4 (hair dryer) are misclassified as each other $33.89 \%$ of the time. Observing Figure 5.6 , it is noted that load 3 and load 4 have very similar switching on transient waveforms after normalization (due to similarity between the tasks of heating elements of electrical circuit inside the hair dryer and air heater), which are much different than the shapes of normalized switching on waveforms of load 1 and load 2. Table 5.1 indicates that in the absence of load 3 or load 4 which have similar waveform shapes, a much higher classification accuracy will be achieved. In the absence of load 3 , the recognition accuracy of $97.037 \%$ is achieved for classification of the remaining electrical devices including load 1 , load 2 , and load4. The same load recognition accuracy of $97.037 \%$ is achieved for classification of load 1 , load2, and 3 in the absence of load 4. It is worth mentioning that, misclassification of load 3 and load 4 can be easily resolved via their steadystate information; load 3 and load 4 are discernible via their steady-state 
real power values (real power values of load 3 and load 4 during their steady-state operation are $1155.6 \mathrm{~W}$ and $359.37 \mathrm{~W}$, respectively).

\subsection{Conclusions}

In our work, A NILM system has been successfully demonstrated for the recognition of electrical devices when operated individually based on their switching on transient behaviour. Electrical devices can be distinguished by their load signatures during transient changes or during their steady-state operation, or some combination of the two. In this chapter, we investigate features that can be extracted from the current and voltage waveforms during transient operation.

In this chapter, experimental results for single device recognition for four common household electrical devices including air heater, hair dryer, computer CRT monitor, and kitchen blender showed that the proposed approach can achieve $\mathbf{8 0 . 8 3 \%}$ classification accuracy. We concluded that switching on transient waveform is useful feature to identify electrical devices and can serve as effective complementary features to steady-state features (such as real power and reactive power that we discussed in Chapter 3). 


\section{Thesis Conclusions and Future}

\section{Recommendations}

Smart homes are becoming valuable and efficient systems in healthcare. However, there is an increasing need for unobtrusive techniques in order to monitor the activities of elderly people within the smart homes. Our main objective for this thesis was to develop a NILM system for smart home purposes. We wanted to design a NILM system in order to monitor the usage of electrical devices within the smart home and validate the chosen techniques experimentally by implementing prototype NILM system. The major conclusions of this thesis work are summarized in Section 6.1 and recommendations for areas of future work are presented in Section 6.2. 


\subsection{Conclusions}

A NILM system has been successfully developed and applied to an actual system involving different common household electrical devices for the recognition of devices when operating individually and simultaneously. The proposed method worked with high device recognition accuracy, demonstrating that steady-state features (i.e., real power and reactive power) and transient features (i.e., switching on transient waveforms) are useful tools to identify electrical devices.

\section{Steady-state Analysis}

This work showed that real power and reactive power are useful steadystate features to identify electrical devices and can serve as effective complementary features to one another. Furthermore, this work showed that we can separate out five different electrical devices when they are operating simultaneously based on their real power consumption. The method can be generalized to more than five electrical devices. Moreover, this work showed that when relatively high powered devices are simultaneously in operation, their power consumptions ends up being lower than when the devices are operated individually. Therefore, while steady-state features such as real power meets the feature-additive criterion theoretically, we may not observe this practically. Our investigation showed that when these two relatively high powered devices (Load 2 and Load 3) are simultaneously in operation, their 
power consumptions ends up being lower than when the devices are operated individually. The result of load identification during the multiple operations of five electrical devices is consistent with our previous hypothesis that the total electrical supply is limited and these devices (electric kettle and coffee maker) probably do not regulate their power supply. As stated in Section 3.2.1, while real power meets the featureadditive criterion theoretically, we may not observe this practically.

One potential solution for resolving this issue is that the voltage level drops when high powered devices are turned on. A dip in the voltage supply was confirmed by examining the RMS voltage when electric kettle and coffee are operating simultaneously $(108.5 \mathrm{~V}, 110 \mathrm{~V}$, and $105 \mathrm{~V}$ represent for the RMS voltage value during individual operation of electric kettle, individual operation of coffee maker, and simultaneous operation of electric kettle and coffee maker, respectively). Ideally, the voltage supply should be constant, so this dip in voltage supply is indicative of a case where the feature additive criterion is not met. It may be possible to derive a correction factor to mitigate issues associated with scenarios where the feature additive criterion is not met practically.

\section{Transient Analysis}

In term of transient analysis, we conclude that different electrical devices can be distinguished based on their switching on transient waveforms. Experimental results showed that electrical devices observed in this work 
have repeatable switching on transient waveforms that can be used as reliable indicators for load identification. To improve recognition accuracy of electrical devices with the same steady-state parameters, i.e., real power and reactive power, transient properties such as turn on transient waveforms can play an important role. Combining transient and steady-state signatures will increase the reliability and accuracy of load identification in NILM systems.

\subsection{Recommendations for future work}

In the future work, our intension is to explore the use of our prototype NILM system in a real world setting, with data that is being collected in an occupied residential smart home. In future work, we will experiment with the features proposed in this paper, along with the addition of other steady-state and transient features in order to determine robustly the operating state of certain classes of devices, such as low power loads, multi-state devices, continuously varying power devices, and devices with different power cycles. Research is also being extended to examine multiple devices in operation simultaneously, along with methods to disaggregate the individual loads from the composite signal. In addition, the work presented here employed a simple classifier (discriminant analysis). Advanced classifiers can likely achieve high classification accuracies even for a larger group of electrical devices and during simultaneous operation of multiple devices, and will be 
more suited for transient analysis (e.g., time delay artificial neural network and hidden Markov models).

We expect to explore new load signatures (features), event detection, and pattern recognition methods in which load identification and usage tracking are increasingly accurate. The best approaches for the highest accuracy in usage monitoring may be obtained by combining the steadystate and transient features to pattern recognition. Finally, we would like to investigate the use of other sources of information acquired from I intelligent sensors within the smart home (such as magnetic switches, infrared motion sensors, Radio Frequency Identification (RFID) networks and video cameras), to enhance the non-intrusive approach to monitor ADL of residents. For example, we could envision correlating the change in light intensity caused by switching on a incandescent lamp on a room with the change in power consumption to infer that this specific change in power was due to an incandescent lamp. 


\section{REFERENCES}

1. Population Division, DESA, United Nations. (March 23, 2010). Magnitude and Speed of Population Ageing [Online]. World Population Ageing 1950-2050. New York: United Nations Publications. pp. 11-13. Available: http://www.un.org/esa/population/publications/worldageing1950205 0/pdf/80chapteril.pdf (Last Accessed: Nov 9, 2011).

2. Health Canada, Division of Aging and Seniors. (2002). Canada's Aging Population. Minister of Public Works and Government Services Canada [Online]. Cat. H39-608/2002E, ISBN 0-662-31821-8. Available: http://dsp-psd.pwgsc.gc.ca/Collection/H39-608-2002E.pdf (Last Accessed: Nov 9, 2011).

3. Robert G. Evans. (1987). Hang Together, or Hang Separately: The Viability of a Universal Health Care System in an Aging Society (University of Toronto Press and Canadian Public Policy) [Online]. Available:

http://www.jstor.org/stable/pdfplus/3550637.pdf?acceptTC=true (Last Accessed: Nov 9, 2011).

4. M. Anderson, K. Parent, "Care in the home: public responsibilityprivate roles?," Paper prepared for the dialogue on health reform. Department of Rehabilitation Medicine, Queen's University, Kingston, Ontario, June 2000. Avallable: http://www.utoronto.ca/hpme/dhr/pdf/Anderson Parentipdf (Last Accessed: Nov 9, 2011).

5. M. Berenguer, M. Giordani, F. Giraud-By, and N. Noury, "Automatic detection of activities of daily living from detecting and classifying electrical events on the residential power line," in Proc. 10th IEEE Int. Conf. e-Health Netw., Appl. Serv. HealthCom, pp. 29-32, 2008.

6. J. Poujaud, N. Noury, and J. E. Lundy., "Identification of inactivity behavior in smart home," In Proc. 30th Annual Int. Conf. of the IEEE Engineering in Medicine and Biology Society EMBS, pp. 2075-2078, 2008. 
7. A. Arcelus, M. Jones, R. Goubran, and F. Knoefel, "Integration of smart home technologies in a health monitoring system for the elderly," in Proc. IEEE AINAW, Niagara Falls, ON, Canada, pp. 820825, May 2007.

8. M. Poland, C.D. Nugent, H. Wang, and L. Chen, "Smart home research: projects and Issue," International Journal of Ambient Computing and Intelligence, 1, 4, pp. 32-45, 2009.

9. Y. R. Zheng, R. A. Goubran, M. El-Tanany, and S. Hongchi, "A microphone array system for multimedia applications with near field signal targets," IEEE Sensors Journal, vol. 5, no. 6, pp. 1395-1406, 2005.

10. H. Chen, R. A. Goubran, and T. Mussivand, "Improving the classification accuracy in electronic noses using multidimension combining," IEEE Sensors Conference, Austria, October 2004.

11. M. Howell Jones, R. A. Goubran, and F. Knoefel, "Reliable respiratory rate estimation from a bed pressure array," Proc. of the 28th IEEE Annual International Conference on Engineering in Medicine and Biology Society, New York, USA, September 2006.

12. M. Howell Jones, A. Arcelus, R.A. Goubran, and F. Knoefel, "A pressure sensitive home environment," IEEE International Workshop on Haptic Audio Visual Environments and their Applications, Ottawa, ON, Canada, 2006.

13. L.K. Norford and S.B. Leeb, "Nonintrusive electrical load monitoring in commercial buildings based on steady state and transient load-detection algorithms," Energy and Buildings, vol. 24, pp. 51-64, 1996.

14. J. Lemmerhirt and R. Blevins, Electric Power Research Institute (EPRI), "Nonintrusive appliance load monitoring system (NIALMS) beta-test results," Palo Alto, Calif. : EPRI, Final Report TR-108419, September 1997.

15. H. Pihala, "Non-intrusive appliance load monitoring system based on a modern kWh-meter", VTT Publications, no. 356, May 1998. 
16. S. Patel, T. Robertson, J. Kientz, M. Reynolds, and G. Abowd, "At the Flick of a Switch: Detecting and Classifying Unique Electrical Events on the Residential Power Line," Proc. Ubicomp07, pp. 271288, Innsbruck, Austria, 2007.

17. M. T. Fitta, "Load Classification and Appliance Fingerprinting for Residential Load Monitoring System," M.S. thesis, Dept. Electrical. Eng., Aalto Univ. School of Science and Tech., Espoo, Finland, 2010.

18. K. Suzuki, S. Inagaki, T. Suzuki, H. Nakamura, and K. Ito, "Nonintrusive appliance load monitoring based on integer programming," in Proc. SICE Annu. Conf., pp. 2742-2747, Aug. 2008.

19. D. Benyoucef, P. Klein, and T. Bier, "Smart Meter with NonIntrusive Load Monitoring for Use in Smart Homes," IEEE International Energy Conference, 2010.

20. Y. H. Lin and M.S. Tsai, "A novel feature extraction method for the development of nonintrusive load monitoring system based on BPANN," International Symposium on Computer Communication Control and Automation (3CA), pp. 215 - 218, 2010.

21. L. Farinaccio and R. Zmeureanu, "Using a pattern recognition approach to disaggregate the total electricity consumption in a house into the major end-uses," Energy and Buildings, no. 30, pp. 245259., 1999.

22. T.R. Sharp, "Nonintrusive load monitoring systems: Considerations for use and potential applications," ACEEE Summer Study on Energy and Efficiency in Buildings, No.2, pp. 241-247, 1994.

23. H.S. Matthews et al., "Automatically disaggregating the total electrical load in residential buildings: A profile of the required solution," Proceedings of Intelligent Computing in Engineering, Plymouth, pp. 381-389, July 2008.

24. S.R. Gomez, D. Culler, and J. Ortiz, "Load disaggregation for increased coverage in a building energy auditing network, "Index of Programs, University of California, Berkeley, 2009. 
25. M. Berges et al., "Training load monitoring algorithms on highly submetered home electricity consumption data," Tsinghua Science \& Technology, vol. 13, pp. 406-411, 2008.

26. H.H. Chang, C.L. Lin, and H.T. Yang, "Load recognition for different loads with the same real power and reactive power in a non-intrusive load-monitoring system," Proceedings of the 12th International Conference on Computer Supported Cooperative Work in Design, Xi'an, China, pp. 1122-1127, 2008.

27. J. Liang et al., "Load signature study-Part I: Basic concept, structure, and methodology," IEEE Transactions on Power Delivery, vol. 25, no. 2, pp. 551-560, April 2010.

28. H. Najmeddine et al., "State of art on load monitoring methods," Proceedings of 2nd IEEE International Conference on Power and Energy," Johor Baharu, Malaysia, pp. 1256-1258, December 1-3, 2008.

29. James Paris, "A Framework For Non-intrusive Load Monitoring and Diagnostics", M.S. thesis, Dept. of Electrical Engineering and Computer Science, Massachusetts Institute of Technology, Boston, MA, USA, 2006.

30. J. Liang et al., "Load signature study-Part II: Disaggregation framework, simulations and applications," IEEE Transactions on Power Delivery, vol. 25, no. 2, pp. 561-569, April 2010.

31. W.K. Lee et al., "Exploration on load signatures," Proceedings of the International Conference on Electrical Engineering, Sapporo, Japan, vol. 2, July 4-8, pp. 690-694, 2004.

32. F. Sultanem, "Using appliance signatures for monitoring residential loads at meter panel level," IEEE Transactions on Power Delivery, Vol. 6, pp.1380-1385, 1991.

33. K.H. Ting et al., "A taxonomy of load signatures for single-phase electric appliances," Paper submitted to IEEE Power Electronics Specialist Conference, June 12-15, 2005. 
34. H.Y. Lam et al., "Building a vector-based load taxonomy using electrical load signatures," Proceedings of the International Conference on Electrical Engineering, July 10-14, 2005.

35. H.Y. Lam, G.S.K. Fung, and W.K. Lee, "A novel method to construct taxonomy of electrical appliances based on load signature," IEEE Transactions on Consumer Electronics, vol. 53, no. 2, pp. 653660, May 2007.

36. C. Laughman et al., "Power signature analysis," IEEE Power and Energy Magazine, vol. 1, no. 2, pp. 56-63, March/April 2003.

37. D.D. Sabin, D.L. Brooks, and A. Sundaram, "Indices for assessing harmonic distortion from power quality measurements: definitions and benchmark data," IEEE Transactions on Power Delivery, vol. 14, no. 2, pp. 489-496, April 1999.

38. D. Srinivasan, W.S. Ng, and A.C. Liew, "Neural-network-based signature recognition for harmonic source identification," IEEE Transactions on Power Delivery, vol. 21, no. 1, pp. 398-405, January 2006.

39. J.G. Roos et al., "Using neural networks for nonintrusive monitoring of industrial electrical loads," Proceedings of the 10th IEEE Instrumentation and Measurement Technology Conference, May 10-12, Hamamatsu, South Africa, vol. 3, pp. 1115-1118, 1994.

40. S.B. Leeb, S.R. Shaw, and J.L. Kirtley Jr., "Transient event detection in spectral envelope estimates for nonintrusive load monitoring," IEEE Transactions on Power Delivery, vol. 10, no. 3, pp. 1200-1210, July 1995.

41. M. Deschizeau, P. Bertrand, A. Anglade, and M. Grimaldi, "A new method for detailed electric consumption of domestic appliances," Energy efficiency in household appliances and lighting, page 419427, 2001.

42. S. Leeb et al., "Development and validation of a transient event detector," AMP Journal of Technology, vol. 3, November 1993. 
43. G.W. Hart, "Nonintrusive appliance load monitoring," Proceedings of the IEEE Computer Applications in Power, vol. 80, no. 12, pp. 1870-1891, December 1992.

44. M. Zeifman and K. Roth, "Nonintrusive Appliance Load Monitoring: Review and Outlook," In Proceedings of the IEEE Conference on Consumer Electronics in Las Vegas, NV. IEEE, 2011.

45. A.J. Bijker, X. Xia, and J. Zhang, "Active power residential nonintrusive appliance load monitoring system," Proceedings of IEEE AFRICON 2009, September 23-25, Nairobi, Kenya, pp. 1-6, 2009.

46. A. Bouloutas, G. W. Hart, and M. Schwartz, "Two Extensions of the Viterbi Algorithm," IEEE Transactions on Information Theory, Vol. 37, pp. 430-436, 1991.

47. G. W. Hart, "Correcting Dependent Errors in Sequences Generated by Finite-State Processes," IEEE Transactions on Information Theory, Vol. 39, pp. 1249-1260, 1993.

48. A. I. Albicki and A. Cole, "Algorithm for Non-Intrusive Identification of Residential Appliances," Circuits and Systems, Proceedings of the IEEE International Symposium on, Vol. III, pp. 338-341, 1998.

49. A. I. Albicki and A. Cole, "Data Extraction for Effective NonIntrusive Identification of Residential Power Loads," IEEE Instrumentation and Measurement Technology Conference, pp. 812815, 1998.

50. A.I. Cole and A. Albicki, "Nonintrusive identification of electrical loads in a three-phase environment based on harmonic content," Proceedings of the 17th IEEE Instrumentation and Measurement Technology Conference, Baltimore, USA, vol. 1, pp. 24-29, 2000.

51. M. L. Marceau and R. Zmeureanu, "Nonintrusive Load Disaggregation Computer Program to Estimate the Energy Consumption of Major End Uses in Residential Buildings," Energy Conversion \& Management, Vol. 41, pp. 1389-1403, 2000. 
52. M. Baranski and J. Voss, "Non-Intrusive Appliance Load Monitoring Based on an Optical Sensor," IEEE Power Tech. Conference, Bologna, 2003.

53. M. Baranski and J. Voss, "Genetic Algorithm for Pattern Detection in NIALM Systems," IEEE International Conference on Systems, Man and Cybernetics, pp. 3462-3468, 2004.

54. M. Baranski and J. Voss, "Detecting Patterns of Appliances from Total Load Data Using a Dynamic Programming Approach," The 4th IEEE International Conference on Data Mining (ICDM'04), pp. 327330, 2004.

55. B. Margossian, Quantum Consulting Inc., "Deriving end use load profiles without end-use metering: results of recent validation studies," Proceedings of the ACEEE 1994 Summer Study on Energy Efficiency in Buildings, pp. 2.217-2.223, 1994.

56. K. D. Lee et al., "Estimation of Variable-Speed-Drive Power Consumption from Harmonic Content," IEEE Transactions on Energy Conversion, Vol. 20, pp. 566-574, 2005.

57. W. Wichakool, A. T. Avestruz, R. W. Cox, and S. B. Leeb, "Modeling and Estimating Current Harmonics of Variable Electronic Loads," IEEE Transactions on Power Electronics, vol. 24, pp. 28032811, 2009.

58. S. R. Shaw, S. B. Leeb, , L. K. Norfold, and R. W. Cox, "Nonintrusive Load Monitoring and Diagnostics in Power Systems," IEEE Transactions on Instrumentation and Measurement, vol. 57, pp. 1445-1454, 2008.

59. S. Gupta, M. S. Reynolds, and S. N. Patel, "ElectriSense: SinglePoint Sensing Using EMI for Electrical Event Detection and Classification in the Home," Conference on Ubiquitous Computing, pp. 139-148, 2010.

60. H. T. Yang, H. H. Chang, and C. L. Lin, "Design a neural network for features selection in non-intrusive monitoring of industrial electrical loads," in Proc. 2007 International Conference on Computer Supported Cooperative Work in Design, pp. 1022-1027, 2007. 
61. H. H. Chang, C. L. Lin, and J. K. Lee, "Load identification in nonintrusive load monitoring using steady-state and turn-on transient energy algorithms," in Proceedings of the 14th International Conference on Computer Supported Cooperative Work in Design (CSCWD '10), pp. 27-32, 2010.

62. H. H. Chang, H. T. Yang, and C.L. Lin, "Load Identification in Neural Networks for a Non-intrusive Monitoring of Industrial Electrical Loads," In: W. Shen, J. Yong, Y. Yang, J. P. Barths, and J. Luo, editors, Computer Supported Cooperative Work in Design IV, volume 5236 of Lecture Notes in Computer Science, pages 664-674. Springer Berlin / Heidelberg, 2008.

63. M. B. Figueiredo, A. de Almeida, B. Ribeiro, and A. Martins, "Extracting features from an electrical signal of a non-intrusive load monitoring system," In: Fyfe, C., Tino, P., Charles, D., Garcia-Osorio, C., Yin, H. (eds.) IDEAL 2010. LNCS, vol. 6283, pp. 210-217. Springer, Heidelberg, 2010.

64. Saba Rahimi, A. D. C. Chan, and Rafik Goubran, "Usage Monitoring of Electrical Devices in a Smart Home," submitted to International Conference of the IEEE Engineering in Medicine and Biology Society, 2011.

65. Magnelab, Inc. Split-Core AC Current sensor SCT-0400 [Online]. Available: http://www.magnelab.com/products/Current-VoltageSensors/Split-Core-AC-Current-Sensor-SCT-0400 (Last Accessed: Nov 14, 2011).

66. Compton Instruments. (2002). Split Core Current Transformers and Transducers [Online]. Available: http://energy.te.com/china/crompton doc/ctsplit.pdf (Last Accessed: Nov 14, 2011).

67. National Instruments Corporation. (2010, April 7). Low-Cost, Bus-Powered Multifunction DAQ for USB [online]. Available: http://sine.ni.com/ds/app/doc/p/id/ds-218/lang/en (Last Accessed: Nov 14, 2011). 
68. National Instruments Corporation. (2011, October 21). BusPowered $M$ Series Multifunction DAQ for USB [online]. Available: http://sine.ni.com/ds/app/doc/p/id/ds-9/lang/en (Last Accessed: Nov 14, 2011).

69. Y. Nakano et al., "Non-Intrusive Electric Appliances Load Monitoring System Using Harmonic Pattern Recognition Performance Test Results at Real Households ," The Fourth International Conference on Energy Efficiency in Domestic Appliances and Lighting (EEDAL) London, pp. 21 - 23, June 2006.

70. A. G. Ruzzelli, C. Nicolas, A. Schoofs, and G. M. P. O'Hare, "Realtime recognition and profiling of devices through a single electricity sensor," in Seventh Annual IEEE Communications Society Conference on Sensor, Mesh, and Ad Hoc Communications and Networks (SECON'10), pp. 1-9, 2010.

71. M. Berges et al., "Learning systems for electric consumption of buildings," Proceedings of the 2009 ASCE International Workshop on Computing in Civil Engineering, Austin, USA, pp. 1-10, 2010.

72. A. D. C. Chan and G. C. Green, "Myoelectric Control Development Toolbox," Proc. Thirtieth Conf. Canadian Medical and Biological Engineering Society, 2007.

73. C.E. Reeg, "Nonintrusive load monitoring for verification and diagnostics" , M.S. thesis, Dept. of Electrical and Computer Engineering, Univ. of Illinois, Urbana, Illinois, 2011.

74. R. O. Duda, P. E. Hart, and D. G. Stork, Pattern Classification, $2^{\text {nd }}$ edition, Wiley-Interscience, 2000.

75. S.R. Kamat, "Fuzzy logic based pattern recognition technique for nonintrusive load monitoring," Proceedings of IEEE Regional 10 Conference, IEEE Press, vol.3, pp. 528-530, Nov. 2004.

76. F. Kupzog, T. Zia and A.A. Zaidi, "Automatic electric load identification in self-configuring micro-grids," Proceedings of IEEE AFRICON 2009, Nairobi, Kenya, pp. 1-5, 2009. 
77. P3 International Corporation. (2007). Kill A Watt Electricity Usage Monitor [Online]. Available: http://www.p3international.com/brochures/p4400.pdf Accessed: Nov 14, 2011).

78. A. Reury, M. Vacher and N. Noury, "SVM-based multi-modal classification of activities of daily living in health smart homes: Sensors, algorithms and first experimental results," IEEE Trans. Inf. Technol. Biomed., vol. 14, no. 2, pp. 274 -283, March 2010.

79. A. Papoulis and S. U. Pillai, Probability, Random Variables and Stochastic Processes. $3^{\text {rd }}$ edition, New York: McGraw-Hill, 1991.

80. R. Lyman Ott, An Introduction to Statistical Methods and Data Analysis, $4^{\text {th }}$ Edition, Duxbury Press, Belmont, CA, USA, 1993

81. Nabney, I.T, Netlab: Algorithms for pattern recognition, Springer, London, 2002. 


\section{Appendix A: DATA AQUISITION DURING THE MULTIPLE OPERATION OF FIVE DIFFERENT ELECTRICAL DEVICES}

Appendix $A$ includes real power waveforms of all the 8 measurement trials presented in Section 4.3.1.

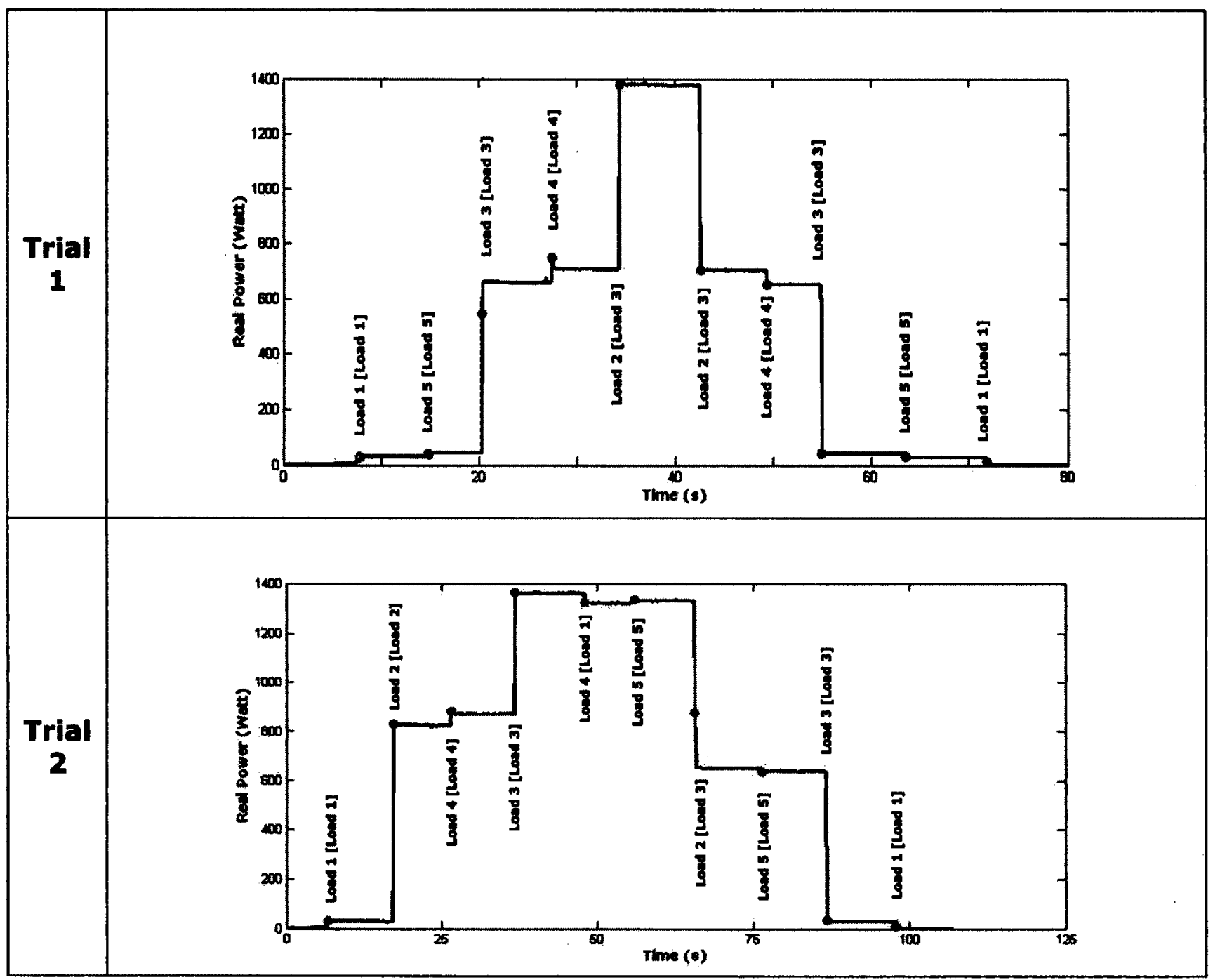




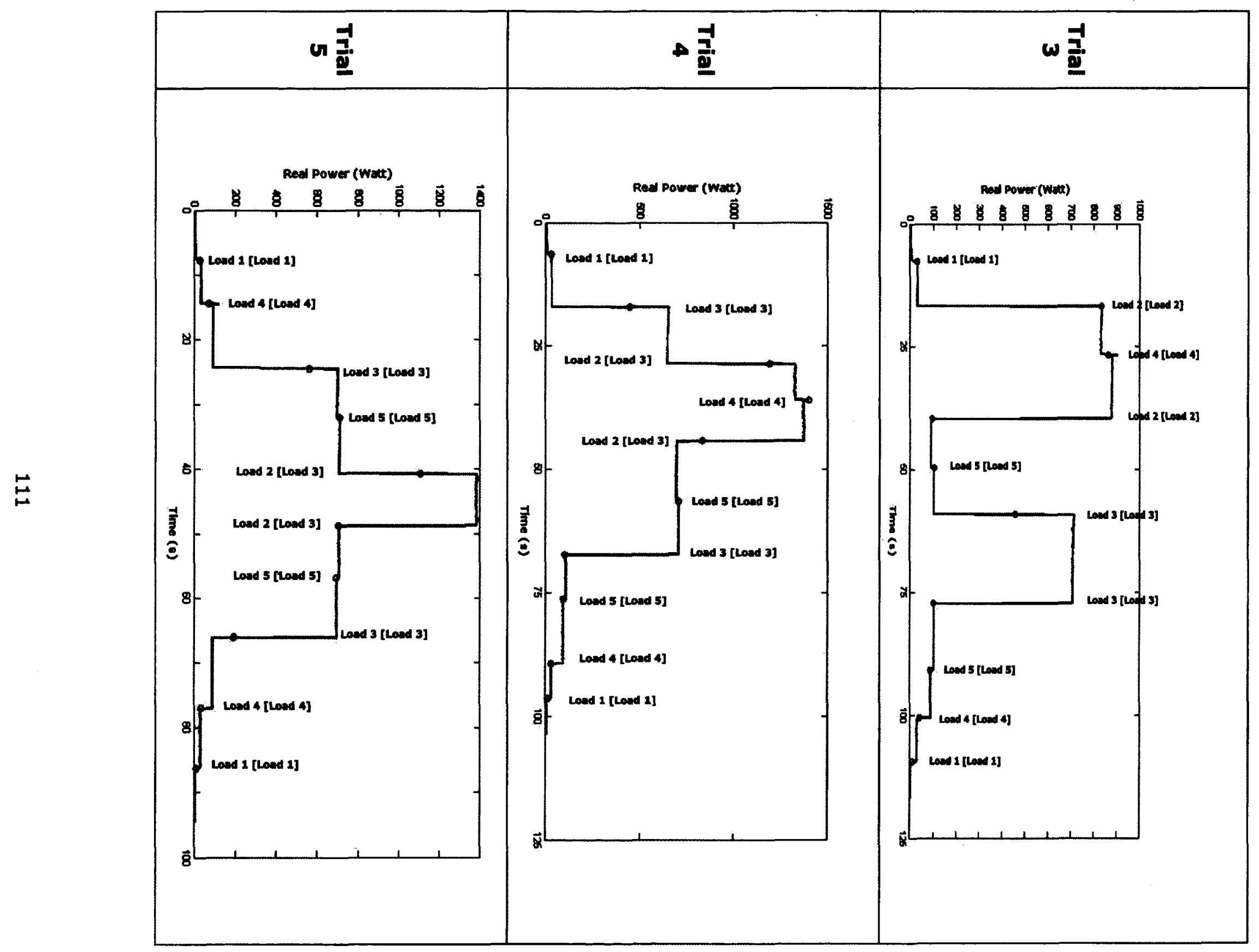




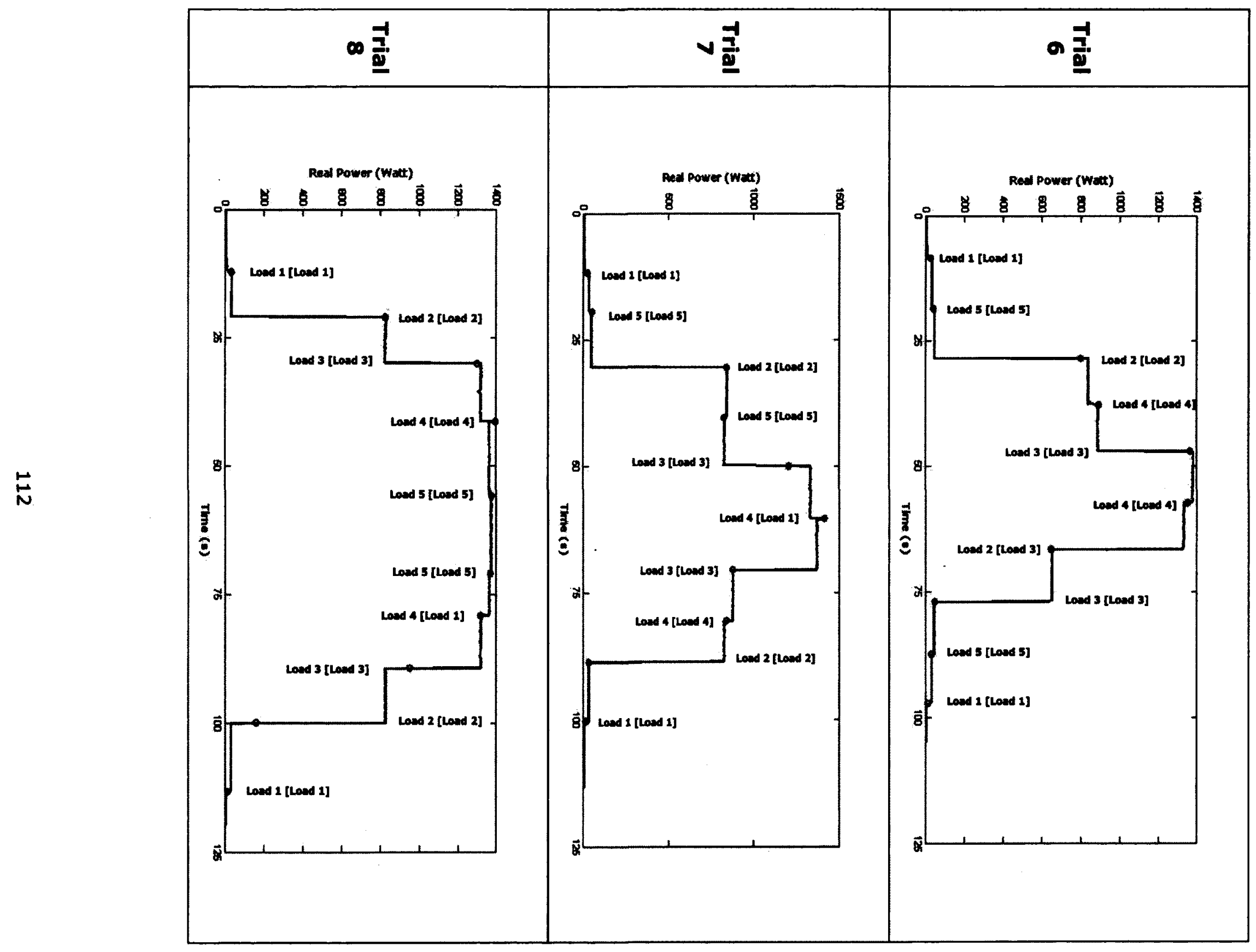




\section{Appendix B: SPECIFICATIONS OF ELECTRICAL DEVICES}

Appendix B includes specifications of all the 11 electrical devices monitored in this project.

\begin{tabular}{|c|c|c|}
\hline & Devices & Specifications \\
\hline 1 & Electric kettle & $\begin{array}{c}\text { Wal Mart } \\
\text { Model: Mk-10P04A } \\
1 \text { litre electric kettle } \\
120 \mathrm{~V}, 60 \mathrm{~Hz}, 1000 \mathrm{~W} \\
\text { Listed: } 3181383\end{array}$ \\
\hline 2 & Microwave & $\begin{array}{c}\text { Danby } \\
\text { Model DMW1158BL } \\
\text { Input: } 120 \mathrm{~V}, 60 \mathrm{~Hz}, \mathrm{AC}, 1.45 \mathrm{KW} \\
\text { Listed } 23 \mathrm{FJ} \\
\text { Operation mode: Reheat }\end{array}$ \\
\hline 3 & Coffee maker & $\begin{array}{c}\text { Toastmaster } \\
\text { Coffemaker model TCM10PWF } \\
120 \text { VAC, } 60 \mathrm{~Hz}, 750 \mathrm{~W} \\
\text { Listed } 442 \mathrm{~A} \\
\text { E6173 }\end{array}$ \\
\hline 4 & Laptop charger & $\begin{array}{c}\text { hp AC adapter } \\
\text { DC359A } \\
\text { Series PPP009H } \\
\text { HP part No. } 380467-003 \\
\text { Listed I.T.E power supply } 9 \mathrm{~F} 64 \\
\text { E143709 } \\
\text { Input: } 100-240 \mathrm{~V} \sim 1.7 \mathrm{~A} 50-60 \mathrm{~Hz} \\
\text { Output: } 18.5 \mathrm{~V} \text { 3.5A } 65 \mathrm{~W} \\
\end{array}$ \\
\hline 5 & Incandescent lamp & $\begin{array}{c}\text { Reveal } 60 \text { Watts, } 120 \text { VAC } \\
\text { Halogen Hungary AO } \\
\text { Light output : } 675 \text { lumens } \\
\text { PC : } 00897\end{array}$ \\
\hline
\end{tabular}




\begin{tabular}{|c|c|c|}
\hline 6 & Computer LCD monitor & $\begin{array}{c}\text { IBM ThinkVision } \\
\text { Model : } 6734-\mathrm{ABO} \\
\text { Serial Number : } 5513222 \\
100-240 \mathrm{~V} \sim 50 / 60 \mathrm{~Hz} 0.7 \mathrm{~A} \\
\text { Canada ICES-003 Class B } \\
\end{array}$ \\
\hline 7 & Fluorescent lamp & $\begin{array}{c}\text { HELICAL } \\
\text { Listed } 6 \mathrm{G} 49 \\
20 \mathrm{~W}, 120 \mathrm{VAC}, 60 \mathrm{~Hz}, 290 \mathrm{~mA} \\
\text { FLE20HT3/2/XL/865 }\end{array}$ \\
\hline 8 & Kitchen blender & $\begin{array}{c}\text { Magic Bullet } \\
\text { Model: MB1001 } \\
120 \text { VAC, Max } 250 \mathrm{~W}, 60 \mathrm{~Hz} \\
\text { Listed: } 33 \mathrm{TJ} \\
\text { E301511 }\end{array}$ \\
\hline 9 & Computer CRT monitor & $\begin{array}{c}\text { SAMSUNG } \\
\text { Sync Master } \\
\text { Model: AN17KSBL } \\
\text { AC 100, } 240 \mathrm{~V}, 60 / 50 \mathrm{~Hz}, 1.2 \mathrm{~A} \\
\text { Listed } 3882 \mathrm{A431} \\
\text { US PAT. No } 5.670972\end{array}$ \\
\hline 10 & Air heater & $\begin{array}{c}\text { Honeywell Air Heater } \\
\text { Listed } 95812 \\
\text { Southborough, MA } 01772 \\
120 \text { VAC, } 60 \mathrm{~Hz}, 12.5 \mathrm{~A}, 1500 \mathrm{~W} \text { TP } \\
\text { Part No. 034-03116-001 } \\
\text { Artwork No. 043-01163-001 } \\
\text { Operational mode: High }\end{array}$ \\
\hline 11 & Hair dryer & $\begin{array}{c}\text { CONAIR IONSHINE } \\
\text { Model: } 157 R I D C \\
125 / 250 \text { VAC, } 60 \mathrm{~Hz}, 1875 \text { Watt } \\
\text { Listed: } 3 \mathrm{H} 32 \\
\text { E47949 }\end{array}$ \\
\hline
\end{tabular}

